\title{
Double Macdonald polynomials as the stable limit of Macdonald superpolynomials
}

\author{
O. Blondeau-Fournier · L. Lapointe · P. Mathieu
}

Received: 9 April 2013 / Accepted: 7 June 2014 / Published online: 10 July 2014

(C) Springer Science+Business Media New York 2014

\begin{abstract}
Macdonald superpolynomials provide a remarkably rich generalization of the usual Macdonald polynomials. The starting point of this work is the observation of a previously unnoticed stability property of the Macdonald superpolynomials when the fermionic sector $m$ is sufficiently large: their decomposition in the monomial basis is then independent of $m$. These stable superpolynomials are readily mapped into bisymmetric polynomials, an operation that spoils the ring structure but drastically simplifies the associated vector space. Our main result is a factorization of the (stable) bisymmetric Macdonald polynomials, called double Macdonald polynomials and indexed by pairs of partitions, into a product of Macdonald polynomials (albeit subject to nontrivial plethystic transformations). As an off-shoot, we note that, after multiplication by a $t$-Vandermonde determinant, this provides explicit formulas for a large class of Macdonald polynomials with prescribed symmetry. The factorization of the double Macdonald polynomials leads immediately to the generalization of basically every elementary properties of the Macdonald polynomials to the double case (norm, kernel, duality, evaluation, positivity, etc). When lifted back to superspace, this validates various previously formulated conjectures in the stable regime. The $q, t$-Kostka coefficients associated to the double Macdonald polynomials are shown to be $q$, $t$-analogs of the dimensions of the irreducible representations of the hyperoctahedral group $B_{n}$.
\end{abstract}

O. Blondeau-Fournier · P. Mathieu ( $\varangle)$

Département de physique, de génie physique et d'optique, Université Laval,

Quebec G1V 0A6, Canada

e-mail: pmathieu@phy.ulaval.ca

O. Blondeau-Fournier

e-mail: olivier.b-fournier.1@ulaval.ca

L. Lapointe

Instituto de Matemática y Física, Universidad de Talca, 2 norte 685, Talca, Chile

e-mail: lapointe@inst-mat.utalca.cl 
Moreover, a Nabla operator on the double Macdonald polynomials is defined, and its action on a certain bisymmetric Schur function can be interpreted as the Frobenius series of a bigraded module of dimension $(2 n+1)^{n}$, a formula again characteristic of the Coxeter group of type $B_{n}$. Finally, as a side result, we obtain a simple identity involving products of four Littlewood-Richardson coefficients.

Keywords Macdonald polynomials - Superpolynomials · Kostka coefficients · Nabla operator $\cdot$ Hyperoctahedral group

\section{Introduction}

\subsection{From superpolynomials to bisymmetric polynomials}

The Macdonald polynomials in superspace, which have been recently introduced in $[6,7]$, provide a combinatorially rich generalization of the Macdonald polynomials. We show in this article that when the fermionic sector is large enough, the Macdonald polynomials in superspace embody a natural form of a bisymmetric extension of the Macdonald polynomials, whose corresponding Kostka and Nabla combinatorics is that of the hyperoctahedral group $B_{n}$ (whereas, it is that of the symmetric group $S_{n}$ in the usual case). This bisymmetric version of Macdonald polynomials, which now depend on two alphabets, will be referred to as double Macdonald polynomials. ${ }^{1}$

Let us put these statements in context by first recalling the definition of a superpolynomial or equivalently said, a polynomial in superspace. In addition to be a polynomial in the usual indeterminates $x_{1}, \ldots, x_{N}$ over $\mathbb{Q}(q, t)$, it is a function of $N$ Grassmannian (also called anticommuting or fermionic) variables $\theta_{1}, \ldots, \theta_{N}$. Such polynomials are said to be symmetric if they are invariant with respect to the simultaneous interchange of $\left(x_{i}, \theta_{i}\right) \leftrightarrow\left(x_{j}, \theta_{j}\right)$. Due to the latter property, if a superpolynomial is symmetric, then it is always a sum of monomials $\theta_{i_{1}} \ldots \theta_{i_{m}}$ multiplied by a polynomial antisymmetric in the variables $x_{i_{1}}, \ldots, x_{i_{m}}$ and symmetric in the remaining ones. The superpolynomials we consider are always bi-homogeneous in the variables $x$ and $\theta$, and their fermionic degree (the degree in $\theta$ ) is denoted $m$. Finally, a symmetric superpolynomial is indexed by a superpartition $\Lambda$ : a pair of partitions $\left(\Lambda^{a} ; \Lambda^{s}\right)$ such that $\Lambda^{a}$ has $m$ distinct parts (the $m$-th one being allowed to be 0 ). We refer to $m$ as the fermionic degree of $\Lambda$ and the sum of all entries of $\Lambda^{a}$ and $\Lambda^{s}$, denoted $|\Lambda|$, as the total (or bosonic) degree. The partitions $\Lambda^{a}$ (resp. $\Lambda^{s}$ ) capture the degree of the antisymmetric (resp. symmetric) polynomial associated to each term $\theta_{i_{1}} \ldots \theta_{i_{m}}$ of the superpolynomial.

To make these comments more concrete, consider the generalization of the monomial and power-sum basis to superspace [6]:

\footnotetext{
${ }^{1}$ Even though the double Macdonald polynomials are connected to the hyperoctahedral group $B_{n}$, it should, however, be kept in mind that they are not the Macdonald polynomials defined on the root lattice of type $B[22,23]$. They are also different from the Macdonald polynomials with hyperoctahedral symmetries constructed in [13].
} 


$$
m_{\Lambda}=\sum_{\sigma \in S_{N}}^{\prime} \theta_{\sigma(1)} \ldots \theta_{\sigma(m)} x_{\sigma(1)}^{\Lambda_{1}} \ldots x_{\sigma(N)}^{\Lambda_{N}}
$$

(where the prime indicates a sum over distinct terms) and

$$
p_{\Lambda}=\tilde{p}_{\Lambda_{1}} \ldots \tilde{p}_{\Lambda_{m}} p_{\Lambda_{m+1}} \ldots p_{\Lambda_{N}}, \quad \text { where } \quad \tilde{p}_{k}=\sum_{i=1}^{N} \theta_{i} x_{i}^{k} \quad \text { and } \quad p_{r}=\sum_{i=1}^{N} x_{i}^{r}
$$

(with $k \geq 0$ and $r \geq 1$ ). If $m=2$ and $N=3$, then we have for instance

$$
m_{(3,1 ; 1)}=\theta_{1} \theta_{2}\left(x_{1}^{3} x_{2}-x_{2}^{3} x_{1}\right) x_{3}+\theta_{1} \theta_{3}\left(x_{1}^{3} x_{3}-x_{3}^{3} x_{1}\right) x_{2}+\theta_{2} \theta_{3}\left(x_{2}^{3} x_{3}-x_{3}^{3} x_{2}\right) x_{1}
$$

and

$$
\begin{aligned}
p_{(3,1 ; 1)}= & \left(\theta_{1} x_{1}^{3}+\theta_{2} x_{2}^{3}+\theta_{3} x_{3}^{3}\right)\left(\theta_{1} x_{1}+\theta_{2} x_{2}+\theta_{3} x_{3}\right)\left(x_{1}+x_{2}+x_{3}\right) \\
= & \left(\theta_{1} \theta_{2}\left(x_{1}^{3} x_{2}-x_{2}^{3} x_{1}\right)+\theta_{1} \theta_{3}\left(x_{1}^{3} x_{3}-x_{3}^{3} x_{1}\right)+\theta_{2} \theta_{3}\left(x_{2}^{3} x_{3}-x_{3}^{3} x_{2}\right)\right) \\
& \times\left(x_{1}+x_{2}+x_{3}\right) .
\end{aligned}
$$

To any superpolynomial, we can associate a bisymmetric polynomial. For a superpolynomial of fermionic degree $m$, this is done by (i) extracting the coefficient of $\theta_{1} \ldots \theta_{m}$ of the superpolynomial, and (ii) dividing the result by the Vandermonde determinant in the variables $x_{1}, \ldots, x_{m}$. By construction, the resulting polynomial is symmetric in both the variables $x=\left(x_{1}, \ldots, x_{m}\right)$ and $y=\left(x_{m+1}, \ldots, x_{N}\right)$. For the examples presented above, this procedure yields $\left(x_{1}^{2} x_{2}+x_{1} x_{2}^{2}\right) x_{3}$ and $\left(x_{1}^{2} x_{2}+x_{1} x_{2}^{2}\right)\left(x_{1}+x_{2}+x_{3}\right)$, respectively.

When we pass to bisymmetric polynomials, as was just described, the natural ring structure of the space of superpolynomials is lost (the ring structure of the space of bisymmetric polynomials is not the right one, especially when considering connections with supersymmetry $[11,12])$. However, in this article, we are only interested in the underlying vector space. As such, it will prove more convenient to work with bisymmetric polynomials. We will then have at our disposal the very powerful (and much better known) language of symmetric function theory.

A bisymmetric polynomial is naturally indexed by a pair of partitions extracted from the superpartition. To be more precise, the correspondence between a superpartition of fermionic degree $m$ and a pair of partitions $\lambda, \mu$ is

$$
\Lambda=\left(\Lambda^{a} ; \Lambda^{s}\right) \leftrightarrow(\lambda, \mu)=\left(\Lambda^{a}-\delta^{m}, \Lambda^{s}\right)
$$

where $\delta^{m}:=(m-1, \ldots, 0)$ stands for the staircase partition. Observe that from (1.2), it is immediate that $\ell(\lambda) \leq m$. With this correspondence, the monomial and power-sums symmetric superpolynomials are then associated to the following bisymmetric polynomials, respectively (see Appendix A for a detailed derivation of this correspondence): 


$$
m_{\Lambda} \longleftrightarrow m_{\lambda, \mu}(x, y):=s_{\lambda}(x) m_{\mu}(y)
$$

and

$$
p_{\Lambda} \quad \longleftrightarrow \quad p_{\lambda, \mu}(x, y):=s_{\lambda}(x) p_{\mu}(x, y)
$$

where $s_{\lambda}, m_{\lambda}$, and $p_{\lambda}$ are, respectively, the Schur, monomial, and power-sum symmetric functions. In the case of $m_{\lambda, \mu}$, the functions in the product depend upon complementary sets of variables, while $p_{\lambda, \mu}$ does not $\left(p_{\mu}(x, y)\right.$ is the usual powersum symmetric functions in the union of the variables $x$ and $y$ ). In the two cases, it is still noteworthy that the corresponding bisymmetric polynomials have such a simple factorization (we stress that $m_{\Lambda}$ and $p_{\Lambda}$ are both functions of the variables $\left.x_{1}, \ldots, x_{N}, \theta_{1}, \ldots, \theta_{N}\right)$.

The bisymmetric polynomials associated to the Macdonald superpolynomials $P_{\Lambda}\left(x_{1}, \ldots, x_{N}, \theta_{1}, \ldots, \theta_{N} ; q, t\right)[6,7]$ will simply be denoted $P_{\Lambda}(x, y ; q, t){ }^{2}$ For reasons to become clear shortly, it is more convenient at this point to keep using superpartitions as indices even for the bisymmetric version of the Macdonald superpolynomials ( $\Lambda$ captures for instance the information on the fermionic degree $m$ which corresponds to the maximal length $\lambda$ can have). We now translate in the language of bisymmetric functions Theorem 1 of [7] which establishes the existence of the Macdonald superpolynomials. It relies on the dominance ordering on superpartitions. For two superpartitions $\Lambda$ and $\Omega$ of fermionic degree $m$ and of the same total degree, it is defined as

$$
\Lambda \geq \Omega \quad \text { iff } \quad \Lambda^{*} \geq \Omega^{*} \quad \text { and } \quad \Lambda^{\circledast} \geq \Omega^{\circledast}
$$

where $\Lambda^{*}, \Lambda^{\circledast}$ are standard partitions (not superpartitions) given by

$$
\Lambda^{*}=\left(\lambda+\delta^{m}\right) \cup \mu \text { and } \Lambda^{\circledast}=\left(\lambda+\delta^{m+1}\right) \cup \mu,
$$

where the order on partitions is the dominance ordering, and where $\Lambda \leftrightarrow(\lambda, \mu)$ in the correspondence (1.2) (the reader is referred to Sect. 2 for the definitions of the dominance ordering and of the operations on partitions $\lambda \cup \mu$ and $\lambda+\mu$ ).

Theorem 1 Let $\Lambda \leftrightarrow(\lambda, \mu)$ be a superpartition of fermionic degree $m$, and set $x=\left(x_{1}, \ldots, x_{m}\right)$ and $y=\left(x_{m+1}, \ldots, x_{N}\right)$. Then, for $N-m \geq|\lambda|+|\mu|$, there exists a unique bisymmetric polynomial $P_{\Lambda}=P_{\Lambda}(x, y ; q, t)$ such that

$$
\begin{aligned}
& \text { (1) } P_{\Lambda}=m_{\lambda, \mu}+\text { lower terms, } \\
& \text { (2) }\left\langle\left\langle P_{\Lambda}, P_{\Omega}\right\rangle_{q, t}=0 \text { if } \Lambda \neq \Omega\right. \text {. }
\end{aligned}
$$

\footnotetext{
${ }^{2}$ The meaning of $P_{\Lambda}$ will be clear from the context, and actually, in the body of the text, it will always refer to the bisymmetric version. The two forms are distinguished by their explicit variable-dependence: either $(x, \theta)$ or $(x, y)$.
} 


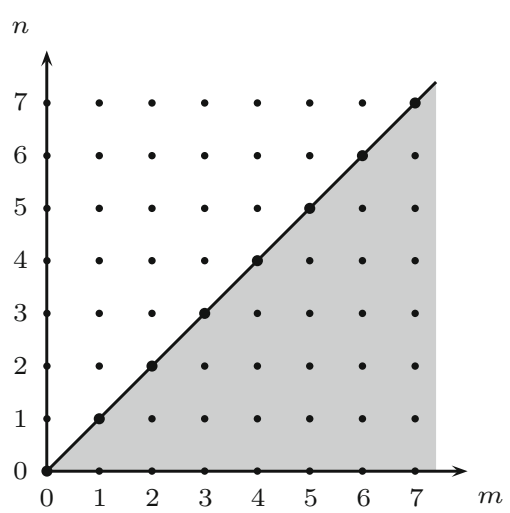

Fig. 1 To each dot corresponds a family of superpolynomials indexed by superpartitions of total degree $\left|\Lambda^{a}\right|+\left|\Lambda^{s}\right|=n+m(m-1) / 2$ and whose component $\Lambda^{a}$ has exactly $m$ parts. The sector $m \geq n$ (indicated in gray) is the domain in which the bisymmetric polynomials are stable. In other words, for a fixed value of $m$, all the points weakly below the diagonal represent equivalent families, for which convenient representatives are the points on the diagonal (larger dots). In the complementary region, the superspace formalism (via the dominance ordering for superpartitions) is mandatory

The dominance ordering on pairs of partitions is such that $(\lambda, \mu) \geq(\omega, \eta)$ iff the corresponding superpartitions $\Lambda$ and $\Omega \leftrightarrow(\omega, \eta)$ are such that $\Lambda \geq \Omega$. The scalar product is defined on the power sums (1.4) as ${ }^{3}$

$$
\left\langle\left\langle p_{\lambda, \mu}, p_{\omega, \eta}\right\rangle_{q, t}=\delta_{\lambda \omega} \delta_{\mu \eta} q^{|\lambda|} z_{\mu}(q, t)\right.
$$

where $z_{\mu}(q, t)$ is given in (2.4).

We stress that the monomial expansion of $P_{\Lambda}$ is independent of $N-m$ (granted that $N-m$ is large enough ${ }^{4}$ ), and thus $N-m$ can be considered infinite. Unexpectedly, a similar independence upon $m$ holds. Let us denote by $n$ the total degree of the pair $(\lambda, \mu)$, that is,

$$
n=|\lambda|+|\mu|
$$

What is remarkable, and totally unanticipated from the supersymmetric point of view, is that even though the ordering (1.5) appears to be highly dependant of $m$, the monomial expansion (1.6) of the bisymmetric polynomial $P_{\Lambda}$ does not depend on $m$ whenever $m \geq n$. This will be referred to as the stable sector of the bisymmetric Macdonald polynomials. This phenomenon is described schematically in Fig. 1. Let us illustrate

\footnotetext{
3 In the expression of the scalar product, we have dropped a factor $(-1 / q)^{m(m-1) / 2}$ which does not affect the orthogonality.

4 In the monomial expansion of $P_{\Lambda}$, the longest indexing superpartition is $\left(\delta^{m} ; 1^{|\lambda|+|\mu|}\right)$, with $\Lambda \leftrightarrow(\lambda, \mu)$. The corresponding monomial is non-zero only if the set of variables $y$ has at least $|\lambda|+|\mu|$ elements (cf. (1.3)). The well definedness of (1.7) thus requires the condition $N \geq m+|\lambda|+|\mu|$. Note that this constraint is trivially satisfied in Theorem 1 of [7] where the number of variables is infinite.
} 
the stability property of $P_{\Lambda}$, where $\Lambda \leftrightarrow(\emptyset,(2))$, by displaying the monomial decompositions when $n=2$ for four different values of $m$ :

$$
\begin{aligned}
P_{(0 ; 2)} & =m_{\emptyset,(2)}+\frac{(1-t)(1+q)}{1-q t} m_{\emptyset,(1,1)}+\frac{(1-t)}{1-q t} m_{(1),(1)} \\
P_{(1,0 ; 2)} & =m_{\emptyset,(2)}+\frac{(1-t)(1+q t)}{1-q t^{2}} m_{\emptyset,(1,1)} \\
P_{(2,1,0 ; 2)} & =m_{\emptyset,(2)}+\frac{(1-t)(1+q t)}{1-q t^{2}} m_{\emptyset,(1,1)} \\
P_{(3,2,1,0 ; 2)} & =m_{\emptyset,(2)}+\frac{(1-t)(1+q t)}{1-q t^{2}} m_{\emptyset,(1,1)} .
\end{aligned}
$$

The first case, for which $m=1<2=n$, does not belong to the stable sector. In the other three cases, one recovers three identical expressions (even though the corresponding monomials depend on different sets of variables).

In the stable sector, it will thus be more natural to index the bisymmetric polynomial $P_{\Lambda}:=P_{\lambda, \mu}$ by the pair of partitions $(\lambda, \mu)$, even more so that in this sector the ordering (1.5) can be replaced by the following dominance ordering on pairs of partitions (cf. Proposition 32 in Appendix B): for $(\lambda, \mu)$ and $(\omega, \eta)$ both of total degree $n$,

$$
\begin{gathered}
(\lambda, \mu) \geq(\omega, \eta) \quad \text { iff } \quad \lambda_{1}+\cdots+\lambda_{i} \geq \omega_{1}+\cdots+\omega_{i} \quad \text { and } \\
|\lambda|+\mu_{1}+\cdots+\mu_{j} \geq|\omega|+\eta_{1}+\cdots+\eta_{j} \quad \forall i, j
\end{gathered}
$$

where it is understood that $\lambda_{k}=0$ if $k>\ell(\lambda)$ (and similarly for $\mu, \omega$ and $\eta$ ).

Because they are labeled by two partitions and, as we will see shortly, they are naturally viewed as a function of two sets of (commuting) variables, the bisymmetric Macdonald polynomials in the stable sector will be called double Macdonald polynomials. Therefore, in the stable sector, Theorem 1 becomes as follows:

Theorem 2 Let $(\lambda, \mu)$ be of total degree $n$. Then, the double Macdonald polynomials $P_{\lambda, \mu}(x, y ; q, t)$, where $x=\left(x_{1}, \ldots, x_{m}\right)$ and $y=\left(x_{m+1}, \ldots, x_{N}\right)$ (with $m \geq n$ and $N-m \geq n)$ are the unique bisymmetric polynomials such that

$$
\begin{aligned}
& P_{\lambda, \mu}(x, y ; q, t)=m_{\lambda, \mu}(x, y)+\sum_{\omega, \eta<\lambda, \mu} c_{\lambda, \mu ; \omega, \eta}(q, t) m_{\omega, \eta}(x, y), \\
& \left\langle\left\langle P_{\lambda, \mu}, P_{\omega, \eta}\right\rangle\right\rangle_{q, t}=0 \text { if }(\lambda, \mu) \neq(\omega, \eta),
\end{aligned}
$$

where the ordering on pairs of partitions and the scalar product are, respectively, defined in (1.8) and (1.7).

If we let $m$ and $N-m$ go to infinity, we obtain double Macdonald functions. We will nevertheless restrict ourselves to the finite case in this article. It should also be stressed that there is no solution to the two conditions (1.9) in the non-stable sector if the ordering (1.8) is used. Therefore, in order to interpolate between the usual and double Macdonald polynomials (corresponding, respectively, to the cases $m=0$ and $m \geq n)$, the construction relying on the super-dominance ordering (1.5) is necessary. 


\subsection{Statement of the main results}

As already pointed out, the bisymmetric version of the monomials and the powersums display a very simple factorization pattern, irrespective of the value of $m$. Such a generic factorization is not expected to be observed for the bisymmetric Macdonald polynomials. However, it turns out that in the stable sector, such a factorization occurs, albeit in a non-obvious way. Using the plethystic notation (reviewed in Sect. 2) with $X=x_{1}+\cdots+x_{m}$ and $Y=x_{m+1}+\cdots+x_{N}$, the factorization of the double Macdonald polynomials reads (cf. Theorem 5)

$$
P_{\lambda, \mu}(x, y ; q, t)=P_{\lambda}^{(q, q t)}\left[X+\frac{q(1-t)}{1-q t} Y\right] P_{\mu}^{(q t, t)}[Y],
$$

where $P_{\lambda}^{(q, t)}(x)$ denotes the usual Macdonald polynomial $P_{\lambda}(x ; q, t)$.

We briefly digress in order to comment on the consequences of this result for the Macdonald polynomials with prescribed symmetry [1]. Let $\mathcal{A}_{t}^{(x)}$ be the $t$-antisymmetrization (or Hecke antisymmetrization) operator acting on the variables $x, \mathcal{S}_{t}^{(y)}$ be the $t$-symmetrization (or Hecke symmetrization) operator acting on the variables $y, \Delta_{t}(x)$ be the $t$-Vandermonde determinant in the variables $x$, and $E_{\gamma}(x, y ; q, t)$ be the non-symmetric Macdonald polynomials in the variables $x_{1}, \ldots, x_{m}, y_{1}, \ldots, y_{N-m}$ (the reader is referred to [1,7] for the relevant definitions). It is known [7] that the double Macdonald polynomial (via the superpolynomial construction) $P_{\lambda, \mu}(x, y ; q, t)$ is related to the Macdonald polynomial with prescribed symmetry $\mathcal{A}_{t}^{(x)} \mathcal{S}_{t}^{(y)} E_{\gamma}(x, y ; q, t)$ through

$$
P_{\lambda, \mu}(x, y ; q, t) \propto \frac{1}{\Delta_{t}(x)} \mathcal{A}_{t}^{(x)} \mathcal{S}_{t}^{(y)} E_{\gamma}(x, y ; q, t),
$$

where $\gamma=\left(\gamma_{1}, \ldots, \gamma_{N}\right)$ is any composition such that $\left(\gamma_{1}, \ldots, \gamma_{m}\right)$ and $\left(\gamma_{m+1}, \ldots, \gamma_{N}\right)$ rearrange to the partitions $\lambda+\delta^{m}$ and $\mu$, respectively, and where $\propto$ means that the result holds up to a constant. The factorization (1.10) translates into a factorization of Macdonald polynomials with prescribed symmetry.

Theorem 3 Let $\gamma=\left(\gamma_{1}, \ldots, \gamma_{N}\right)$ be a composition such that $\gamma_{1}, \ldots, \gamma_{m}$ are all distinct and such that $m, N-m \geq|\gamma|-m(m-1) / 2$. Then, the Macdonald polynomial with prescribed symmetry $\mathcal{A}_{t}^{(x)} \mathcal{S}_{t}^{(y)} E_{\gamma}(x, y ; q, t)$ is such that

$$
\mathcal{A}_{t}^{(x)} \mathcal{S}_{t}^{(y)} E_{\gamma}(x, y ; q, t) \propto \Delta_{t}(x) P_{\lambda}^{(q, q t)}\left[X+\frac{q(1-t)}{1-q t} Y\right] P_{\mu}^{(q t, t)}[Y],
$$

where $\lambda+\delta^{m}$ and $\mu$ are the partitions corresponding, respectively, to the rearrangements of $\left(\gamma_{1}, \ldots, \gamma_{m}\right)$ and $\left(\gamma_{m+1}, \ldots, \gamma_{N}\right)$.

The theorem means that if we take any non-symmetric Macdonald polynomials indexed by a composition of sufficiently low degree, $t$-antisymmetrize with respect to the first $m$ variables, and $t$-symmetrize with respect to the remaining ones, then 
the result is either zero (if there are repeated entries in the first $m$ entries of the composition) or, quite amazingly, a $t$-Vandermonde determinant times a product of two Macdonald polynomials! We should add that the degenerate case $\lambda=\emptyset$ of (1.11) was already known [1, Proposition 2].

Returning to our main line, we stress that the factorization (1.10) offers the royal road to study the properties of the double Macdonald polynomials. To a large extent, this study amounts to lifting to the factorized form, known properties of the usual Macdonald polynomials (cf. [21, Chapter VI]). In this way, we readily obtain the norm, the duality, and the evaluation (respectively, given by Corollary 7, Proposition 8 , and Corollary 10).

As a side result, we point out an interesting consequence of the duality: a simple identity involving products of four Littlewood-Richardson coefficients (see Proposition 9). For any partitions $\lambda, \mu, \nu$, and $\omega$, we have

$$
\sum_{\gamma, \eta, \sigma, \tau}(-1)^{|\tau|} c_{\tau^{\prime} \nu}^{\gamma} c_{\gamma \eta}^{\lambda} c_{\eta \mu}^{\sigma} c_{\sigma \tau}^{\omega}=\delta_{\lambda \nu} \delta_{\mu \omega}
$$

where $\gamma, \eta, \sigma, \tau$ run over all partitions and $c_{\mu \nu}^{\lambda}$ is the corresponding LittlewoodRichardson coefficient.

Implementing the plethystic substitutions $X \mapsto X$ and $X+Y \mapsto(X+Y) /(1-t)$ on the r.h.s. of (1.10) defines the modified double Macdonald polynomials:

$$
H_{\lambda, \mu}(x, y ; q, t)=H_{\lambda}^{(q, q t)}[X+q Y] H_{\mu}^{(q t, t)}[t X+Y],
$$

where $H_{\lambda}^{(q, t)}[X]=J_{\lambda}^{(q, t)}[X /(1-t)]$ is the modified Macdonald polynomial $\left(J_{\lambda}^{(q, t)}(x)\right.$ is the integral form of the Macdonald polynomial $\left.P_{\lambda}^{(q, t)}(x)\right)$. The expansion of $H_{\lambda, \mu}(x, y ; q, t)$ in terms of the Schur functions $s_{\lambda, \mu}(x, y)=s_{\lambda}(x) s_{\mu}(y)$ defines the double Kostka coefficients $K_{\kappa, \gamma} \lambda, \mu(q, t)$ :

$$
H_{\lambda, \mu}(x, y ; q, t)=\sum_{\kappa, \gamma} K_{\kappa, \gamma} \lambda, \mu(q, t) s_{\kappa, \gamma}(x, y) .
$$

We show that $K_{\kappa, \gamma} \lambda, \mu(1,1)$ is equal to the dimension of the irreducible representation of $B_{n}$ indexed by the pairs of partitions $(\kappa, \gamma)$ (see Proposition 11). This is the first genuine contact with the hyperoctahedral group. We then show that the basic properties of the double Kostka coefficients, namely their positivity and symmetries, are immediate consequences of the factorization (1.12) and the related properties of the usual $q, t$-Kostkas (cf. Proposition 12 and Corollary 14).

Next, we define a deformation of the Nabla operator [4], denoted $\nabla^{B}$, whose eigenfunctions are $H_{\lambda, \mu}\left(x, y ; q, t^{-1}\right)$ and whose eigenvalues are given by a specific ratio of two double Kostkas. Somewhat surprisingly, we can evaluate the Schur expansion (which happens to be positive) of $\nabla^{B} s_{\emptyset,(n)}$ exactly (cf. Proposition 17). From the ensuing expression, we deduce the following two results which will provide our most 
significant connection with the hyperoctahedral group:

$$
\begin{aligned}
\left\langle\nabla^{B} s_{\emptyset ;(n)}, s_{\emptyset ;(n)}\right\rangle_{B} & =\frac{1}{(q t)^{\left(\begin{array}{c}
n \\
2
\end{array}\right)}}\left[\begin{array}{c}
2 n \\
n
\end{array}\right]_{q, t} \\
\left\langle\nabla^{B} s_{\emptyset,(n)}, p_{\emptyset,\left(1^{n}\right)}\right\rangle_{B} & =\left(\frac{[n+1]_{q, t}+[n]_{q, t}}{(q t)^{(n-1) / 2}}\right)^{n},
\end{aligned}
$$

where $\langle\cdot, \cdot\rangle_{B}$ is the hyperoctahedral version of the Hall scalar product, and where

$$
\begin{gathered}
{[k]_{q, t}=\frac{q^{k}-t^{k}}{q-t}, \quad\left[\begin{array}{c}
2 n \\
n
\end{array}\right]_{q, t}=\frac{[2 n]_{q, t} !}{[n]_{q, t} ![n]_{q, t} !}, \quad \text { with }} \\
{[k]_{q, t} !=[k]_{q, t}[k-1]_{q, t} \cdots[1]_{q, t} .}
\end{gathered}
$$

In [17], Haiman conjectured that for every Coxeter group $W$, there exists a doubly graded quotient ring $R^{W}$ of the coinvariant ring $C^{W}$ whose Hilbert series $\operatorname{Hilb}_{q, t}\left(R^{W}\right)$ satisfies

$$
\operatorname{Hilb}_{t^{-1}, t}\left(R^{W}\right)=t^{-h r / 2}\left([h+1]_{t}\right)^{r},
$$

where $h$ represents the Coxeter number of $W$ and $r$ its rank. The existence of such modules has been demonstrated in [15] using the representation theory of Cherednik algebras. When $W=B_{n}$, this formula specializes to

$$
\operatorname{Hilb}_{t^{-1}, t}\left(R^{B_{n}}\right)=\left(\frac{[2 n+1]_{t}}{t^{n}}\right)^{n},
$$

which is exactly the r.h.s of (1.15) when $q=t^{-1}$. Furthermore, it is known [2] that the alternating component of $R^{B_{n}}$ is given by the $t$-Catalan for the reflection group $B_{n}$

$$
t^{-n^{2}} \prod_{i=1}^{n} \frac{\left(1-t^{2 i+2 n}\right)}{\left(1-t^{2 i}\right)}
$$

which again corresponds to the r.h.s of (1.14) specialized to $q=t^{-1}$. It is thus natural to surmise that

$$
\operatorname{Frob}_{t^{-1}, t}\left(R^{B_{n}}\right) \sim \nabla_{q=t^{-1}}^{B} s_{\emptyset ;(n)},
$$

where the symbol $\sim$ means that the equality holds up to a relabeling of the indices (to ensure that the trivial module appears only at bidegree $(0,0)$, we probably need, in view of Corollary 20, to relabel the indexing pair of partitions as $s_{\lambda, \mu} \mapsto s_{\lambda^{\prime}, \mu^{\prime}}$ ).

As for the generic $q, t$-case, it does not appear that $\operatorname{Frob}_{q, t}\left(R^{B_{n}}\right) \sim \nabla^{B} s_{\emptyset ;(n)}$ (at least not with the natural grading stemming from [15]). Already in the $n=2$ case, the $B_{2}$-Catalan is $[5]_{q, t}+q t[1]_{q, t}$ (see for instance [24]) while (1.14) gives $\left([5]_{q, t}+q^{2} t^{2}[1]_{q, t}\right) / q t$ (which is in some sense a homogenization of the $B_{2}$-Catalan). 


\subsection{Outline}

Apart from the brief Sect. 2, reviewing the notation and the Conclusion, the article is essentially divided in two parts. The first one, Sect. 3, is devoted to the study of the double Macdonald polynomials defined in Theorem 2. The pivotal result is the establishment of an equivalence between two scalar products, from which the factorization form (1.10) is deduced. The rest of the section is concerned with the derivation of direct consequences of this main formula. The second main part, Sect. 4, is concerned with the investigation of generalizations of the Nabla operator. After reviewing how this operator is defined in the usual case, we present our heuristic approach that yields, among other things, the results mentioned in the previous subsection. In the short Conclusion, we reassert a new role for the Macdonald superpolynomials as the precise and fully explicit objects that interpolates between the usual and double versions of Macdonald polynomials.

Four appendices complete this article. Appendix A contains the details of the statements made in the starting subsection concerning the transformation of the monomials and the power sums, from superspace to bisymmetric functions. Appendix B is mainly concerned with the proof of the equivalence between the two dominance orderings (1.5) and (1.8) in the stable sector. This crucial result is relegated to an appendix because it is fairly technical and also because in disguised form, it is probably known. As discussed in the Conclusion, the results demonstrated here for the double Macdonald polynomials can be readily lifted to superspace. In Appendix C, we show that certain conjectured results in superspace are now validated in the stable sector, by matching the statements in $[6,7]$ with those of the present paper. Tables of $B_{n}$ Kostka coefficients up to $n=3$ are presented in Appendix D.

\section{Definitions}

A partition $\lambda=\left(\lambda_{1}, \lambda_{2}, \ldots\right)$ of degree $|\lambda|=\sum_{i} \lambda_{i}$ is a vector of non-negative integers such that $\lambda_{i} \geq \lambda_{i+1}$ for $i=1,2, \ldots$. The length $\ell(\lambda)$ of $\lambda$ is the number of non-zero entries of $\lambda$. Each partition $\lambda$ has an associated Ferrers diagram with $\lambda_{i}$ lattice squares in the $i$ th row, from the top to bottom. Any lattice square in the Ferrers diagram is called a cell (or simply a square), where the cell $(i, j)$ is in the $i$ th row and $j$ th column of the diagram. The conjugate $\lambda^{\prime}$ of a partition $\lambda$ is the partition whose diagram is obtained by reflecting the diagram of $\lambda$ about the main diagonal. Given a cell $s=(i, j)$ in $\lambda$, we let

$$
a_{\lambda}(s)=\lambda_{i}-j, \quad \text { and } \quad l_{\lambda}(s)=\lambda_{j}^{\prime}-i
$$

The quantities $a_{\lambda}(s)$ and $l_{\lambda}(s)$ are, respectively, called the arm length and leg length. We will also need their co-version:

$$
a_{\lambda}^{\prime}(s)=j-1, \quad \text { and } \quad l_{\lambda}^{\prime}(s)=i-1 .
$$

We say that the diagram $\mu$ is contained in $\lambda$, denoted $\mu \subseteq \lambda$, if $\mu_{i} \leq \lambda_{i}$ for all $i$. We also let $\lambda+\mu$ be the partitions whose entries are $(\lambda+\mu)_{i}=\lambda_{i}+\mu_{i}$, and 
$\lambda \cup \mu$ be the partition obtained by reordering the entries of the concatenation of $\lambda$ and $\mu$. The dominance ordering on partitions is such that $\lambda \geq \mu$ iff $|\lambda|=|\mu|$ and $\lambda_{1}+\ldots+\lambda_{i} \geq \mu_{1}+\ldots+\mu_{i}$ for all $i$.

The Macdonald polynomials $P_{\lambda}(x ; q, t)$, in the variables $x=x_{1}, x_{2}, \ldots$, are characterized by the two conditions: [21]

$$
\begin{aligned}
& \text { (1) } P_{\lambda}(x ; q, t)=m_{\lambda}+\text { lower terms, } \\
& \text { (2) }\left\langle P_{\lambda}, P_{\mu}\right\rangle_{q, t}=0 \text { if } \lambda \neq \mu
\end{aligned}
$$

The triangular decomposition refers to the dominance order on partitions, and the $m_{\lambda}$ 's are the monomial symmetric functions:

$$
m_{\lambda}=\sum_{\sigma \in S_{N}}^{\prime} x_{\sigma(1)}^{\lambda_{1}} \ldots x_{\sigma(N)}^{\lambda_{N}}
$$

where the prime indicates a sum over distinct terms $x_{\sigma(1)}^{\lambda_{1}} \cdots x_{\sigma(N)}^{\lambda_{N}}$. The orthogonality relation is defined in the power-sum basis $p_{\lambda}=p_{\lambda_{1}} \ldots p_{\lambda_{\ell}}$, with $p_{r}=\sum_{i \geq 1} x_{i}^{r}$, as

$$
\begin{aligned}
\left\langle p_{\lambda}, p_{\mu}\right\rangle_{q, t} & =\delta_{\lambda \mu} z_{\lambda}(q, t) \quad \text { where } \\
z_{\lambda}(q, t) & =z_{\lambda} \prod_{i=1}^{\ell(\lambda)} \frac{1-q^{\lambda_{i}}}{1-t^{\lambda_{i}}}=\prod_{i \geq 1} i^{n_{\lambda}(i)} n_{\lambda}(i) !\left(\prod_{i=1}^{\ell(\lambda)} \frac{1-q^{\lambda_{i}}}{1-t^{\lambda_{i}}}\right),
\end{aligned}
$$

$n_{\lambda}(i)$ being the number of parts in $\lambda$ equal to $i$. We stress the notational distinction between the scalar product $\langle\cdot, \cdot\rangle_{q, t}$ and its bisymmetric version $\left\langle\langle\cdot, \cdot\rangle_{q, t}\right.$.

The Jack polynomials $P_{\lambda}(x ; \alpha)$ and Schur functions $s_{\lambda}$ can be defined, respectively, as the limits $q=t^{\alpha}, t \rightarrow 1$ and $q=t$ of the Macdonald polynomials. In the latter case, the scalar product (2.4) reduces to the Hall scalar product $\left\langle p_{\lambda}, p_{\mu}\right\rangle=\delta_{\lambda \mu} z_{\lambda}$ which is such that $\left\langle s_{\lambda}, s_{\mu}\right\rangle=\delta_{\lambda \mu}$.

We will use the language of $\lambda$-rings (or plethysms) $[3,20]$. The power-sum $p_{i}$ acts on the ring of rational functions in $x_{1}, \ldots, x_{N}, q, t$ with coefficients in a field $\mathbb{K}$ (usually taken to be $\mathbb{Q}$ ) as

$$
p_{i}\left[\frac{\sum_{\alpha} c_{\alpha} u_{\alpha}}{\sum_{\beta} d_{\alpha} v_{\beta}}\right]=\frac{\sum_{\alpha} c_{\alpha} u_{\alpha}^{i}}{\sum_{\beta} d_{\alpha} v_{\beta}^{i}},
$$

where $c_{\alpha}$ and $d_{\beta} \in \mathbb{K}$ and where $u_{\alpha}, v_{\beta}$ are monomials in $x_{1}, \ldots, x_{N}, q, t$. Since the power-sums form a basis of the ring of symmetric functions, this action extends uniquely to an action of the ring of symmetric functions on the ring of rational functions in $x_{1}, \ldots, x_{N}, q, t$ with coefficients in $\mathbb{K}$. In this notation, a symmetric function $f(x)$ is equal to $f[X]$, where $x=\left(x_{1}, x_{2}, \ldots, x_{N}\right)$ and $X=x_{1}+x_{2}+\cdots+x_{N}$. 


\section{Double Macdonald polynomials}

\subsection{A remarkable factorization property}

As indicated in the Introduction, we are interested in the stable bisymmetric version of the Macdonald polynomials, namely the double Macdonald polynomials defined in Theorem 2 . The stability property is captured by the condition $m \geq n$. In other words, if $m$ and $N-m$ are sufficiently large $(\geq|\lambda|+|\mu|)$, then the bisymmetric Macdonald polynomial $P_{\lambda, \mu}(x, y ; q, t)$ stabilizes, in the sense that its monomial expansion becomes independent of $m$ and $N$. Within the stability sector, we can thus let $m \rightarrow \infty$ and $N-m \rightarrow \infty$, and obtain "double Macdonald functions" indexed by two infinite sets of indeterminates $x=x_{1}, x_{2}, \ldots$ and $y=y_{1}, y_{2}, \ldots$ (corresponding, respectively, to $x_{1}, \ldots, x_{m}$ and $x_{m+1}, \ldots, x_{N}$ in the limit $m \rightarrow \infty$ and $N-m \rightarrow \infty$ ). The distinction between "functions" and "polynomials" will not be necessary here and we shall always consider that our alphabets are finite.

From now on, we will use the "plethystic" notation which is central to the derivation of our results (see Sect. 2). For the remainder of this article, $X$ and $Y$ will stand, respectively, for $X=x_{1}+x_{2}+\cdots+x_{m}$ and $Y=y_{1}+y_{2}+\cdots+y_{N-m}=$ $x_{m+1}+\cdots+x_{N}$.

We first prove that the scalar product (1.7) can be rewritten in a much more convenient way for our purposes. It is in some sense the strongest result of this section.

Lemma 4 If $m \geq|\lambda|+|\mu|$ and $N-m \geq|\lambda|+|\mu|$, then the scalar product (1.7) is equal to the scalar product $\langle\langle\cdot, \cdot\rangle\rangle^{\prime}$ defined as

$$
\begin{aligned}
& \left\langle\left\langle p_{\lambda}\left[X+\frac{q(1-t)}{1-q t} Y\right] p_{\mu}[Y], p_{\nu}\left[X+\frac{q(1-t)}{1-q t} Y\right] p_{\omega}[Y]\right\rangle\right\rangle^{\prime} \\
& =\delta_{\lambda \nu} \delta_{\mu \omega} q^{|\lambda|} z_{\lambda}(q, q t) z_{\mu}(q t, t) .
\end{aligned}
$$

Proof The scalar product (1.7) can be rewritten as

$$
\begin{aligned}
\left\langle\left\langle s_{\lambda}[X] p_{\mu}[X+Y], s_{\nu}[X] p_{\omega}[X+Y]\right\rangle\right\rangle_{q, t} & =q^{|\lambda|}\left\langle s_{\lambda}, s_{\nu}\right\rangle\left\langle p_{\mu}, p_{\omega}\right\rangle_{q, t} \\
& =q^{|\lambda|} \delta_{\lambda \nu} \times \delta_{\mu \omega} z_{\mu}(q, t) .
\end{aligned}
$$

Equation (3.2) is then equivalent to

$$
\left\langle\left\langle p_{\lambda}[X] p_{\mu}[X+Y], p_{\nu}[X] p_{\omega}[X+Y]\right\rangle\right\rangle_{q, t}=\delta_{\lambda \nu} \delta_{\mu \omega} q^{|\lambda|} z_{\lambda} z_{\mu}(q, t)
$$

given that the Hall scalar product is such that $\left\langle p_{\lambda}, p_{\nu}\right\rangle=\delta_{\lambda \nu} z_{\lambda}$. Notice that by homogeneity, the extra factor $q^{|\lambda|}$ can be carried from (3.2) to (3.3).

We now show that the scalar products $\langle\langle\cdot, \cdot\rangle\rangle_{q, t}$ and $\langle\langle\cdot, \cdot\rangle\rangle^{\prime}$ coincide. Let

$p_{r}\left[X+\frac{q(1-t)}{1-q t} Y\right]=u_{r}, \quad p_{r}[Y]=v_{r}, \quad p_{r}[X]=\bar{u}_{r}, \quad$ and $\quad p_{r}[X+Y]=\bar{v}_{r}$. 
The quantities $u_{r}$ and $v_{r}$ are related to $\bar{u}_{r}$ and $\bar{v}_{r}$ through

$$
u_{r}=p_{r}\left[\frac{(1-q) X}{1-q t}+\frac{q(1-t)(X+Y)}{1-q t}\right]=\frac{1-q^{r}}{1-q^{r} t^{r}} \bar{u}_{r}+\frac{q^{r}\left(1-t^{r}\right)}{1-q^{r} t^{r}} \bar{v}_{r}
$$

and

$$
v_{r}=p_{r}[-X+X+Y]=-\bar{u}_{r}+\bar{v}_{r} .
$$

With respect to the scalar product $\langle\langle\cdot, \cdot\rangle\rangle^{\prime}$, we have (using $\partial_{x}=\frac{\partial}{\partial x}$ )

$$
u_{r}^{\perp^{\prime}}=\frac{1-q^{r}}{1-q^{r} t^{r}} q^{r} r \partial_{u_{r}} \quad \text { and } \quad v_{r}^{\perp^{\prime}}=\frac{1-q^{r} t^{r}}{1-t^{r}} r \partial_{v_{r}},
$$

while with respect to the scalar product $\langle\langle\cdot, \cdot\rangle\rangle_{q, t}$, we have

$$
\bar{u}_{r}^{\perp}=q^{r} r \partial_{\bar{u}_{r}} \quad \text { and } \quad \bar{v}_{r}^{\perp}=\frac{1-q^{r}}{1-t^{r}} r \partial_{\bar{v}_{r}}
$$

where $h^{\perp}$ and $h^{\perp^{\prime}}$ are defined, respectively, such that $\left\langle\langle h f, g\rangle_{q, t}=\left\langle\left\langle f, h^{\perp} g\right\rangle_{q, t}\right.\right.$ and $\langle\langle h f, g\rangle\rangle^{\prime}=\left\langle\left\langle f, h^{\perp^{\prime}} g\right\rangle\right\rangle^{\prime}$. Given that $\left\{u_{\lambda} v_{\mu}\right\}_{\lambda, \mu}$ is a basis of the space of bisymmetric functions of a given total degree $n=|\lambda|+|\mu|$, the lemma will follow if we can show that

$$
\left\langle\left\langle u_{\lambda} v_{\mu}, u_{v} v_{\omega}\right\rangle\right\rangle^{\prime}=\left\langle\left\langle u_{\lambda} v_{\mu}, u_{v} v_{\omega}\right\rangle_{q, t},\right.
$$

or equivalently that

$$
\left\langle\left\langle u_{\emptyset} v_{\emptyset}, u_{\lambda}^{\perp^{\prime}} v_{\mu}^{\perp^{\prime}} u_{v} v_{\omega}\right\rangle\right\rangle^{\prime}=\left\langle\left\langle u_{\emptyset} v_{\emptyset}, u_{\lambda}^{\perp} v_{\mu}^{\perp} u_{v} v_{\omega}\right\rangle_{q, t} .\right.
$$

In order to do so, it suffices to compare the recursions induced by $u_{r}$ and $v_{r}$; repeated applications of the recursions will, by homogeneity, either lead to zero or

$$
\left\langle\left\langle u_{\emptyset} v_{\emptyset}, u_{\emptyset} v_{\emptyset}\right\rangle_{q, t}=\left\langle\left\langle u_{\emptyset} v_{\emptyset}, u_{\emptyset} v_{\emptyset}\right\rangle\right\rangle^{\prime}=1\right.
$$

and the result will follow.

Note that the conditions $m \geq|\lambda|+|\mu|$ and $N-m \geq|\lambda|+|\mu|$ ensure that $u_{1}, u_{2}, \ldots$ and $v_{1}, v_{2}, \ldots$ can be considered independent (and similarly for $\bar{u}_{1}, \bar{u}_{2}, \ldots$ and $\bar{v}_{1}, \bar{v}_{2}, \ldots$ ). Observe also from (3.4) and (3.5) that $\partial_{\bar{u}_{r}}$ and $\partial_{\bar{v}_{r}}$ commute with $u_{s}$ and $v_{s}$ if $r \neq s$. Now let $\lambda=\left(r^{k}\right) \cup \hat{\lambda}, \mu=\left(r^{m}\right) \cup \hat{\mu}, v=\left(r^{\ell}\right) \cup \hat{v}$, and $\omega=\left(r^{n}\right) \cup \hat{\omega}$, where $\hat{\lambda}, \hat{\mu}$, $\hat{v}$, and $\hat{\omega}$ do not contain parts of size $r$. On the one hand, we have from (3.6)

$$
\begin{aligned}
\left\langle\left\langle u_{\lambda} v_{\mu}, u_{\nu} v_{\omega}\right\rangle\right\rangle^{\prime} & =\left\langle\left\langle u_{r}^{k} v_{r}^{m} u_{\hat{\lambda}} v_{\hat{\mu}}, u_{r}^{\ell} v_{r}^{n} u_{\hat{\nu}} v_{\hat{\omega}}\right\rangle\right\rangle^{\prime}=\left\langle\left\langle u_{r}^{k-1} v_{r}^{m} u_{\hat{\lambda}} v_{\hat{\mu}}, u_{r}^{\perp^{\prime}} u_{r}^{\ell} v_{r}^{n} u_{\hat{\nu}} v_{\hat{\omega}}\right\rangle\right\rangle^{\prime} \\
& =q^{r} r \ell \frac{1-q^{r}}{1-q^{r} t^{r}}\left\langle\left\langle u_{r}^{k-1} v_{r}^{m} u_{\hat{\lambda}} v_{\hat{\mu}}, u_{r}^{\ell-1} v_{r}^{n} u_{\hat{\nu}} v_{\hat{\omega}}\right\rangle\right\rangle^{\prime} .
\end{aligned}
$$


On the other hand, using (3.4), (3.7), and the chain rule for derivatives, e.g.,

$$
\frac{\partial}{\partial \bar{u}_{r}}=\frac{\partial u_{r}}{\partial \bar{u}_{r}} \frac{\partial}{\partial u_{r}}+\frac{\partial v_{r}}{\partial \bar{u}_{r}} \frac{\partial}{\partial v_{r}},
$$

we get

$$
\begin{aligned}
\left\langle\left\langle u_{\lambda} v_{\mu}, u_{\nu} v_{\omega}\right\rangle_{q, t}\right. & =\left\langle\left\langle u_{r}^{k} v_{r}^{m} u_{\hat{\lambda}} v_{\hat{\mu}}, u_{r}^{\ell} v_{r}^{n} u_{\hat{\nu}} v_{\hat{\omega}}\right\rangle_{q, t}\right. \\
= & \frac{1-q^{r}}{1-q^{r} t^{r}}\left\langle\left\langle u_{r}^{k-1} v_{r}^{m} u_{\hat{\lambda}} v_{\hat{\mu}}, \bar{u}_{r}^{\perp} u_{r}^{\ell} v_{r}^{n} u_{\hat{\nu}} v_{\hat{\omega}}\right\rangle_{q, t}\right. \\
& +\frac{q^{r}\left(1-t^{r}\right)}{1-q^{r} t^{r}}\left\langle\left\langle u_{r}^{k-1} v_{r}^{m} u_{\hat{\lambda}} v_{\hat{\mu}}, \bar{v}_{r}^{\perp} u_{r}^{\ell} v_{r}^{n} u_{\hat{\nu}} v_{\hat{\omega}}\right\rangle_{q, t}\right. \\
= & \left(\frac{1-q^{r}}{1-q^{r} t^{r}}\right)^{2} q^{r} r \ell\left\langle\left\langle u_{r}^{k-1} v_{r}^{m} u_{\hat{\lambda}} v_{\hat{\mu}}, u_{r}^{\ell-1} v_{r}^{n} u_{\hat{\nu}} v_{\hat{\omega}}\right\rangle\right\rangle_{q, t} \\
& -\frac{1-q^{r}}{1-q^{r} t^{r}} q^{r} r n\left\langle\left\langle u_{r}^{k-1} v_{r}^{m} u_{\hat{\lambda}} v_{\hat{\mu}}, u_{r}^{\ell} v_{r}^{n-1} u_{\hat{\nu}} v_{\hat{\omega}}\right\rangle_{q, t}\right. \\
& +\left(q^{r} \frac{1-t^{r}}{1-q^{r} t^{r}}\right)^{2} r \ell \frac{1-q^{r}}{1-t^{r}}\left\langle\left\langle u_{r}^{k-1} v_{r}^{m} u_{\hat{\lambda}} v_{\hat{\mu}}, u_{r}^{\ell-1} v_{r}^{n} u_{\hat{\nu}} v_{\hat{\omega}}\right\rangle_{q, t}\right. \\
& +q^{r} \frac{1-t^{r}}{1-q^{r} t^{r}} r n \frac{1-q^{r}}{1-t^{r}}\left\langle\left\langle u_{r}^{k-1} v_{r}^{m} u_{\hat{\lambda}} v_{\hat{\mu}}, u_{r}^{\ell} v_{r}^{n-1} u_{\hat{\nu}} v_{\hat{\omega}}\right\rangle_{q, t}\right. \\
= & q^{r} r \ell \frac{1-q^{r}}{1-q^{r} t^{r}}\left\langle\left\langle u_{r}^{k-1} v_{r}^{m} u_{\hat{\lambda}} v_{\hat{\mu}}, u_{r}^{\ell-1} v_{r}^{n} u_{\hat{\nu}} v_{\hat{\omega}}\right\rangle_{q, t} .\right.
\end{aligned}
$$

Comparing (3.8) and (3.9), we see that the two $u_{r}$ recursions coincide.

We now do the same for the $v_{r}$ recursions. From (3.7), we find

$$
\begin{aligned}
\left\langle\left\langle u_{\lambda} v_{\mu}, u_{\nu} v_{\omega}\right\rangle\right\rangle^{\prime} & =\left\langle\left\langle u_{r}^{k} v_{r}^{m} u_{\hat{\lambda}} v_{\hat{\mu}}, u_{r}^{\ell} v_{r}^{n} u_{\hat{v}} v_{\hat{\omega}}\right\rangle\right\rangle^{\prime}=\left\langle\left\langle u_{r}^{k} v_{r}^{m-1} u_{\hat{\lambda}} v_{\hat{\mu}}, v_{r}^{\perp^{\prime}} u_{r}^{\ell} v_{r}^{n} u_{\hat{v}} v_{\hat{\omega}}\right\rangle\right\rangle^{\prime} \\
& =\frac{1-q^{r} t^{r}}{1-t^{r}} n r\left\langle\left\langle u_{r}^{k} v_{r}^{m-1} u_{\hat{\lambda}} v_{\hat{\mu}}, u_{r}^{\ell} v_{r}^{n-1} u_{\hat{\nu}} v_{\hat{\omega}}\right\rangle\right\rangle^{\prime},
\end{aligned}
$$

while using (3.5) and (3.7), we get

$$
\begin{aligned}
\left\langle\left\langle u_{\lambda} v_{\mu}, u_{v} v_{\omega}\right\rangle_{q, t}=\right. & \left\langle\left\langle u_{r}^{k} v_{r}^{m} u_{\hat{\lambda}} v_{\hat{\mu}}, u_{r}^{\ell} v_{r}^{n} u_{\hat{v}} v_{\hat{\omega}}\right\rangle_{q, t}\right. \\
= & \left\langle\left\langle u_{r}^{k} v_{r}^{m-1} u_{\hat{\lambda}} v_{\hat{\mu}}, \bar{v}_{r}^{\perp} u_{r}^{\ell} v_{r}^{n} u_{\hat{v}} v_{\hat{\omega}}\right\rangle_{q, t}\right. \\
& -\left\langle\left\langle u_{r}^{k} v_{r}^{m-1} u_{\hat{\lambda}} v_{\hat{\mu}}, \bar{u}_{r}^{\perp} u_{r}^{\ell} v_{r}^{n} u_{\hat{v}} v_{\hat{\omega}}\right\rangle_{q, t}\right. \\
= & \frac{1-q^{r}}{1-t^{r}} r \ell q^{r} \frac{1-t^{r}}{1-q^{r} t^{r}}\left\langle\left\langle u_{r}^{k} v_{r}^{m-1} u_{\hat{\lambda}} v_{\hat{\mu}}, u_{r}^{\ell-1} v_{r}^{n} u_{\hat{v}} v_{\hat{\omega}}\right\rangle_{q, t}\right. \\
& +\frac{1-q^{r}}{1-t^{r}} r n\left\langle\left\langle u_{r}^{k} v_{r}^{m-1} u_{\hat{\lambda}} v_{\hat{\mu}}, u_{r}^{\ell} v_{r}^{n-1} u_{\hat{v}} v_{\hat{\omega}}\right\rangle_{q, t}\right. \\
& -q^{r} r \ell \frac{1-q^{r}}{1-q^{r} t^{r}}\left\langle\left\langle u_{r}^{k} v_{r}^{m-1} u_{\hat{\lambda}} v_{\hat{\mu}}, u_{r}^{\ell-1} v_{r}^{n} u_{\hat{v}} v_{\hat{\omega}}\right\rangle_{q, t}\right. \\
& +q^{r} r n\left\langle\left\langle u_{r}^{k} v_{r}^{m-1} u_{\hat{\lambda}} v_{\hat{\mu}}, u_{r}^{\ell} v_{r}^{n-1} u_{\hat{v}} v_{\hat{\omega}}\right\rangle\right\rangle_{q, t}
\end{aligned}
$$




$$
=\frac{1-q^{r} t^{r}}{1-t^{r}} r n\left\langle\left\langle u_{r}^{k} v_{r}^{m-1} u_{\hat{\lambda}} v_{\hat{\mu}}, u_{r}^{\ell} v_{r}^{n-1} u_{\hat{v}} v_{\hat{\omega}}\right\rangle_{q, t} .\right.
$$

The two $v_{r}$ recursions are thus also seen to coincide, which completes the proof of the assertion that the scalar products $\langle\langle\cdot, \cdot\rangle\rangle^{\prime}$ and $\langle\langle\cdot, \cdot\rangle\rangle_{q, t}$ are equal.

We now have all the tools to establish our key result: the double Macdonald polynomials have a totally unexpected, albeit rather non-trivial, decomposition into a product of two ordinary Macdonald polynomials. As mentioned in the introduction, the special case $\lambda=\emptyset$ of the factorization is essentially contained in [1].

Theorem 5 If $m \geq|\lambda|+|\mu|$ and $N-m \geq|\lambda|+|\mu|$, then

$$
P_{\lambda, \mu}(x, y ; q, t)=P_{\lambda}^{(q, q t)}\left[X+\frac{q(1-t)}{1-q t} Y\right] P_{\mu}^{(q t, t)}[Y]
$$

where $P_{\lambda}^{(q, t)}(x)$ stands for the usual Macdonald polynomial $P_{\lambda}(x ; q, t)$.

It should be commented that although the factorization of Theorem 5 seems highly asymmetric in $X$ and $Y$, the plethystic substitution (3.31) will transform it into the much more symmetrical expression (3.32).

Proof We need to show that the products of Macdonald polynomials appearing on the r.h.s of (3.10) are unitriangular when expanded in the monomial basis and orthogonal with respect to the scalar product $\left\langle\langle\cdot, \cdot\rangle_{q, t}\right.$ defined in (1.7).

The products of Macdonald polynomials $P_{\lambda}^{(q, q t)}\left[X+\frac{q(1-t)}{1-q t} Y\right] P_{\mu}^{(q t, t)}[Y]$ are (basically by definition) orthogonal with respect to the scalar product $\langle\langle\cdot, \cdot\rangle\rangle^{\prime}$ defined in (3.1) (notice that by homogeneity the extra factor $q^{|\lambda|}$ does not have any effect on the orthogonality). By Lemma 4, the orthogonality is then immediate.

We now show the unitriangularity of $P_{\lambda}^{(q, q t)}\left[X+\frac{q(1-t)}{1-q t} Y\right] P_{\mu}^{(q t, t)}[Y]$ in the monomial basis. By triangularity of the Macdonald polynomials, both in the Schur and monomial bases, we get

$$
P_{\lambda}^{(q, q t)}\left[X+\frac{q(1-t)}{1-q t} Y\right] P_{\mu}^{(q t, t)}[Y]=\sum_{\nu \leq \lambda} * s_{\nu}\left[X+\frac{q(1-t)}{1-q t} Y\right] \sum_{\omega \leq \mu} * m_{\omega}[Y]
$$

where $*$ stands for some irrelevant coefficients (that we will keep denoting $*$ ). We have

$$
\begin{aligned}
s_{\nu}\left[X+\frac{q(1-t)}{1-q t} Y\right] & =\sum_{\rho \subseteq v, \gamma \subseteq v} c_{\rho \gamma}^{v} s_{\rho}[X] s_{\gamma}\left[\frac{q(1-t)}{1-q t} Y\right] \\
& =\sum_{\rho \subseteq v, \sigma:|\sigma|+|\rho|=|v|} * s_{\rho}[X] s_{\sigma}[Y] .
\end{aligned}
$$

Note that in the last equation, we only used $|\sigma|=|\gamma|$ and $|\gamma|+|\rho|=|\nu|$ (there is no triangularity when $s_{\gamma}\left[\frac{q(1-t)}{1-q t} Y\right]$ is expanded in the $s_{\sigma}[Y]$ basis). Moreover, it is an 
elementary fact that

$$
s_{\sigma}[Y] m_{\omega}[Y]=m_{\sigma+\omega}[Y]+\sum_{\beta<\sigma+\omega} * m_{\beta}[Y]
$$

Therefore,

$$
P_{\lambda}^{(q, q t)}\left[X+\frac{q(1-t)}{1-q t} Y\right] P_{\mu}^{(q t, t)}[Y]=\sum_{\nu \leq \lambda, \omega \leq \mu} \sum_{\rho \subseteq \nu, \sigma:|\sigma|+|\rho|=|\nu|} \sum_{\beta \leq \sigma+\omega} * s_{\rho}[X] m_{\beta}[Y]
$$

From $\lambda \geq v$ and $v \supseteq \rho$, we obtain

$$
\lambda_{1}+\cdots+\lambda_{i} \geq v_{1}+\cdots+v_{i} \geq \rho_{1}+\cdots+\rho_{i},
$$

while from $\mu \geq \omega,|\rho|+|\sigma|=|\nu|=|\lambda|$, and $\omega+\sigma \geq \beta$, we get

$$
\begin{gathered}
|\lambda|+\mu_{1}+\cdots+\mu_{i} \geq|\rho|+|\sigma|+\omega_{1}+\cdots+\omega_{i} \geq|\rho|+\omega_{1} \\
+\sigma_{1}+\cdots+\omega_{i}+\sigma_{i} \geq|\rho|+\beta_{1}+\cdots+\beta_{i},
\end{gathered}
$$

which proves the triangularity. The unitriangularity is immediate from the unitriangularity of the Macdonald polynomials when expanded in the Schur or the monomial basis.

For later references, we state explicitly the Jack limit.

Corollary 6 If $m \geq|\lambda|+|\mu|$ and $N-m \geq|\lambda|+|\mu|$, then in the limit $q=t^{\alpha}, t \rightarrow 1$, we obtain

$$
P_{\lambda, \mu}(x, y ; \alpha)=\lim _{q=t^{\alpha}, t \rightarrow 1} P_{\lambda, \mu}(x, y ; q, t)=P_{\lambda}^{(\alpha /(\alpha+1))}\left[X+\frac{1}{\alpha+1} Y\right] P_{\mu}^{(\alpha+1)}[Y],
$$

where $P_{\lambda}^{(\alpha)}(x)$ stands for the usual Jack polynomial $P_{\lambda}(x ; \alpha)$.

Since the Jack superpolynomials (see for instance [9]) can be obtained from the Macdonald superpolynomials $P_{\Lambda}(x, \theta ; q, t)$ in the limit $q=t^{\alpha}, t \rightarrow 1$, this corollary yields the factorized expression of the double Jack polynomials which correspond to the stable limit of the bisymmetric polynomials associated to the Jack superpolynomials.

It is important to stress that in (3.11), the plethystic notation is such that $p_{r}$ acts on the ring of rational functions in the variables $x_{1}, \ldots, x_{m}, y_{1}, \ldots, y_{N-m}$ over the field 
$\mathbb{Q}(\alpha)$. Consequently, $\alpha$ is not affected by the plethysm ${ }^{5}$, that is,

$$
p_{r}\left[X+\frac{1}{\alpha+1} Y\right]=p_{r}[X]+\frac{1}{\alpha+1} p_{r}[Y] .
$$

\subsection{Norm}

Using Theorem 5, we obtain rather directly the expression for the norm of the double Macdonald polynomials.

Corollary 7 The norm of the double Macdonald polynomial $P_{\lambda, \mu}(x, y ; q, t)$ is

$$
\left\langle\left\langle P_{\lambda, \mu}(x, y ; q, t), P_{\lambda, \mu}(x, y ; q, t)\right\rangle\right\rangle_{q, t}=q^{|\lambda|} b_{\lambda}(q, q t)^{-1} b_{\mu}(q t, t)^{-1}=: b_{\lambda, \mu}(q, t)^{-1},
$$

where

$$
b_{\lambda}(q, t)=\prod_{s \in \lambda} \frac{1-q^{a(s)} t^{l(s)+1}}{1-q^{a(s)+1} t^{l(s)}} .
$$

Proof Let $Z=X+\frac{q(1-t)}{1-q t} Y$. In Lemma 4, we have shown that the scalar product (1.7) is equivalent to the scalar product

$$
\begin{aligned}
\left\langle\left\langle p_{\lambda}[Z] p_{\mu}[Y], p_{\nu}[Z] p_{\omega}[Y]\right\rangle\right\rangle^{\prime} & =\delta_{\lambda \nu} \delta_{\mu \omega} q^{|\lambda|} z_{\lambda}(q, q t) z_{\mu}(q t, t) \\
& =q^{|\lambda|}\left\langle p_{\lambda}, p_{\nu}\right\rangle_{q, q t} \times\left\langle p_{\mu}, p_{\omega}\right\rangle_{q t, t} .
\end{aligned}
$$

Therefore, the factorized form of $P_{\lambda, \mu}(x, y ; q, t)$ also implies a factorization of its scalar product:

$$
\begin{aligned}
\left\langle\left\langle P_{\lambda, \mu}(x, y ; q, t), P_{\lambda, \mu}(x, y ; q, t)\right\rangle\right\rangle_{q, t} & =q^{|\lambda|}\left\langle P_{\lambda}(x ; q, q t), P_{\lambda}(x ; q, q t)\right\rangle_{q, q t} \\
& \times\left\langle P_{\mu}(x ; q t, t), P_{\mu}(x ; q t, t)\right\rangle_{q t, t},
\end{aligned}
$$

and the result follows from the norm of the Macdonald polynomials [21, VI.4.11]

$$
\left\langle P_{\lambda}(x ; q, t), P_{\lambda}(x ; q, t)\right\rangle_{q, t}=b_{\lambda}(q, t)^{-1} .
$$

5 This is easily seen that

$$
\begin{aligned}
\operatorname{pr}\left[X+\frac{1}{\alpha+1} Y\right] & =\lim _{\substack{q=t^{\alpha} \\
t \rightarrow 1}} \operatorname{pr}\left[X+\frac{q(1-t)}{1-q t} Y\right]=\lim _{\substack{q=t^{\alpha} \\
t \rightarrow 1}}\left(\operatorname{pr}_{r}[X]+\frac{q^{r}\left(1-t^{r}\right)}{1-q^{r} t^{r}} p_{r}[Y]\right) \\
& =p_{r}[X]+\frac{1}{\alpha+1} p_{r}[Y] .
\end{aligned}
$$


If we define the normalized version of the double Macdonald polynomials as

$$
Q_{\lambda, \mu}(x, y ; q, t)=b_{\lambda, \mu}(q, t) P_{\lambda, \mu}(x, y ; q, t),
$$

where $b_{\lambda, \mu}(q, t)=\left\langle\left\langle P_{\lambda, \mu}, P_{\lambda, \mu}\right\rangle_{q, t}^{-1}\right.$ was defined in (3.12), then we obtain

$$
\left\langle\left\langle Q_{\lambda, \mu}(x, y ; q, t), P_{\nu, \omega}(x, y ; q, t)\right\rangle_{q, t}=\delta_{\lambda \nu} \delta_{\mu \omega} .\right.
$$

\subsection{Kernel}

The form (1.7) of the scalar product leads to a natural generalization of the Macdonald kernel. Let $u$ (resp. $v$ ) be the union of the alphabets $\left(x_{1}, x_{2}, \ldots\right)$ and $\left(y_{1}, y_{2}, \ldots\right)$ (resp. $\left(z_{1}, z_{2}, \ldots\right)$ and $\left.\left(w_{1}, w_{2}, \ldots\right)\right)$. Defining

$$
\Pi:=\Pi(u, v ; q, t)=\prod_{i, j} \frac{\left(t u_{i} v_{j} ; q\right)_{\infty}}{\left(u_{i} v_{j} ; q\right)_{\infty}} \prod_{k, l} \frac{1}{1-q^{-1} x_{k} z_{l}},
$$

we obtain by standard manipulations that

$$
\begin{aligned}
\Pi & =\left\{\sum_{\mu} z_{\mu}(q, t)^{-1} p_{\mu}(x, y) p_{\mu}(z, w)\right\}\left\{\sum_{\lambda} \frac{1}{q^{|\lambda|}} s_{\lambda}(x) s_{\lambda}(z)\right\} \\
& =\sum_{\lambda, \mu} z_{\lambda, \mu}(q, t)^{-1} p_{\lambda, \mu}(x, y) p_{\lambda, \mu}(z, w) .
\end{aligned}
$$

The duality (3.13) then implies that

$$
\Pi=\sum_{\lambda, \mu} P_{\lambda, \mu}(x, y ; q, t) Q_{\lambda, \mu}(z, w ; q, t) .
$$

\subsection{Specializations}

In [6], a picture describing the various specializations of the Macdonald polynomials in superspace was presented. Figure 2 gives the corresponding picture in the case of the double Macdonald polynomials. In the figure, there are two Hall-Littlewood limits $\left(P_{\lambda, \mu}(x, y ; t)\right.$ and $\left.\bar{P}_{\lambda, \mu}(x, y ; t)\right)$, one Jack limit $\left(P_{\lambda, \mu}^{(\alpha)}(x, y)\right)$ and a one-parameter Schur limit $\left(s_{\lambda, \mu}(x, y ; t)=P_{\lambda, \mu}(x, y ; t, t)\right)$ that specializes to the corresponding limits of the Hall-Littlewood and Jack limits. Given the factorized form (3.10) of $P_{\lambda, \mu}(x, y ; q, t)$, we can give each of these limits explicitly. The Jack limit was presented in (3.11). The Hall-Littlewood limits are

$P_{\lambda, \mu}(x, y ; t)=s_{\lambda}(x) P_{\mu}(y ; t)$ and $\bar{P}_{\lambda, \mu}(x, y ; t)=s_{\lambda}[X+(1-1 / t) Y] P_{\mu}(y ; 1 / t)$,

where $P_{\mu}(y ; t)$ is the Hall-Littlewood polynomial (the limit $q=0$ of the corresponding Macdonald polynomial). Finally, the Schur limit is 
Fig. 2 Limiting cases of the double Macdonald polynomials

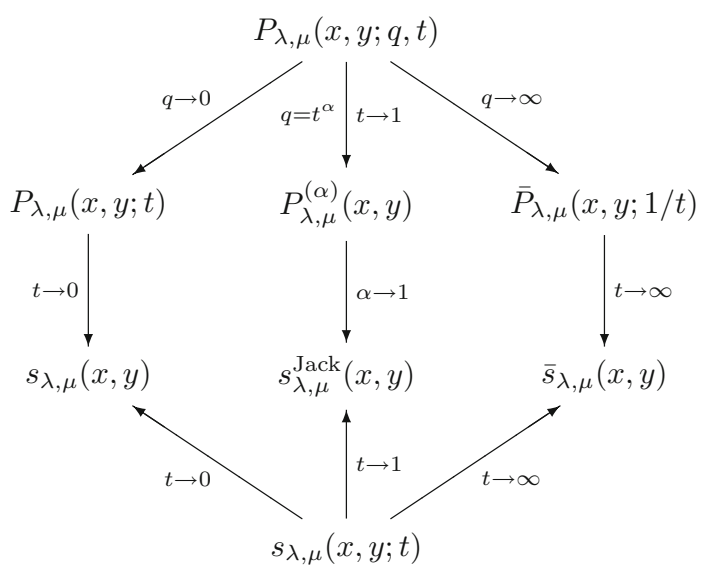

$$
s_{\lambda, \mu}(x, y ; t)=P_{\lambda}^{\left(t, t^{2}\right)}\left[X+\frac{t}{1+t} Y\right] P_{\mu}^{\left(t^{2}, t\right)}[Y],
$$

which specializes to

$$
\begin{aligned}
& s_{\lambda, \mu}(x, y)=s_{\lambda}(x) s_{\mu}(y), \quad s_{\lambda, \mu}^{\mathrm{Jack}}(x, y)=P_{\lambda}^{(1 / 2)}\left[X+\frac{1}{2} Y\right] P_{\mu}^{(2)}[Y] \quad \text { and } \\
& \bar{s}_{\lambda, \mu}(x, y)=s_{\lambda}[X+Y] s_{\mu}(y),
\end{aligned}
$$

where we recall that $P^{(\alpha)}(y)$ is the Jack polynomial. Not considered in Fig. 2 are the limits $q=1$ and $t=1$, which give, respectively,

$$
e_{\lambda, \mu}(x, y)=e_{\lambda}[X+Y] s_{\mu}(y) \quad \text { and } \quad m_{\lambda, \mu}(x, y)=s_{\lambda}(x) m_{\mu}(y)
$$

the analogs of the elementary and monomial symmetric functions.

\subsection{Duality}

Let $\omega_{X}$ be the standard involution

$$
\omega_{X} p_{r}[X]=(-1)^{r-1} p_{r}[X]=(-1)^{r} p_{r}[-X]
$$

which is such that $\omega_{X} s_{\lambda}[X]=s_{\lambda^{\prime}}[X]$. The involution $\omega=\omega_{X} \circ \omega_{Y}$ is such that for any elements $a(q, t)$ and $b(q, t)$ of $\mathbb{Q}(q, t)$, we have

$$
\begin{aligned}
\omega p_{r}[a(q, t) X+b(q, t) Y] & =a\left(q^{r}, t^{r}\right) \omega_{X} p_{r}[X]+b\left(q^{r}, t^{r}\right) \omega_{Y} p_{r}[Y] \\
& =(-1)^{r-1} p_{r}[a(q, t) X+b(q, t) Y] .
\end{aligned}
$$

Hence, $\omega$ acts as the usual involution on symmetric functions in any alphabet made out of a combination of $X$ and $Y$. 
Let us now define the more general automorphism $\omega_{q, t}^{B}$ as

$$
\begin{aligned}
& \omega_{q, t}^{B} p_{r}[X+Y]=(-1)^{r-1} \frac{1-q^{-r}}{1-t^{-r}} p_{r}[X+Y]=\omega p_{r}\left[\frac{t}{q}\left(\frac{1-q}{1-t}\right)(X+Y)\right], \\
& \omega_{q, t}^{B} p_{r}[X]=(-1)^{r} t^{r} p_{r}[X]=\omega p_{r}[-t X] .
\end{aligned}
$$

Clearly, the inverse of $\omega_{q, t}^{B}$ is given by

$$
\left(\omega_{q, t}^{B}\right)^{-1}=\left(\frac{q}{t}\right)^{n} \omega_{t^{-1}, q^{-1}}^{B}
$$

Proposition 8 The following dualities hold:

$$
\begin{aligned}
\omega_{q, t}^{B} P_{\lambda, \mu}(x, y ; q, t) & =Q_{\mu^{\prime}, \lambda^{\prime}}\left(x, y ; t^{-1}, q^{-1}\right), \\
\omega_{q, t}^{B} Q_{\lambda, \mu}(x, y ; q, t) & =(t / q)^{n} P_{\mu^{\prime}, \lambda^{\prime}}\left(x, y ; t^{-1}, q^{-1}\right),
\end{aligned}
$$

where $|\lambda|+|\mu|=n$. In particular (see (3.13)),

$$
\left\langle\left\langle\omega_{q, t}^{B} P_{\mu^{\prime}, \lambda^{\prime}}(x, y ; q, t), P_{\nu, \omega}\left(x, y ; t^{-1}, q^{-1}\right)\right\rangle_{t^{-1}, q^{-1}}=\delta_{\lambda \nu} \delta_{\mu \omega} .\right.
$$

Observe that as expected, the $B_{n}$-analog of the conjugation sends the pair of partitions $\mu, \lambda$ to the pair of partitions $\lambda^{\prime}, \mu^{\prime}$.

Proof We will prove (3.16). Relation (3.15) will then imply (3.17).

First, from the factorized form of the double Macdonald polynomial (3.10), we have

$$
\begin{aligned}
P_{\mu^{\prime}, \lambda^{\prime}}\left(x, y ; t^{-1}, q^{-1}\right) & =P_{\mu^{\prime}}^{\left(t^{-1},(q t)^{-1}\right)}\left[X+\frac{\left(q^{-1}\right)\left(1-t^{-1}\right) Y}{1-(q t)^{-1}}\right] P_{\lambda^{\prime}}^{\left((q t)^{-1}, q^{-1}\right)}[Y] \\
& =P_{\mu^{\prime}}^{(t, q t)}\left[X+\frac{(1-q) Y}{1-q t}\right] P_{\lambda^{\prime}}^{(q t, q)}[Y]
\end{aligned}
$$

where, in the second equality, we used the symmetry $P_{\lambda}^{\left(q^{-1}, t^{-1}\right)}[X]=P_{\lambda}^{(q, t)}[X]$. The duality can now be computed explicitly. We have

$$
\begin{aligned}
& \omega_{q, t}^{B} P_{\lambda, \mu}(x, y ; q, t) \\
& \quad=\omega P_{\lambda}^{(q, q t)}\left[t \frac{1-q}{1-q t} Y\right] \omega P_{\mu}^{(q t, t)}\left[\left(\frac{t}{q}\right) \frac{1-q t}{1-t}\left(X+\frac{Y(1-q)}{1-q t}\right)\right] \\
& \quad=t^{|\lambda|} b_{\lambda^{\prime}}(q t, q) P_{\lambda^{\prime}}^{(q t, q)}[Y]\left(\frac{t}{q}\right)^{|\mu|} b_{\mu^{\prime}}(t, q t) P_{\mu^{\prime}}^{(t, q t)}\left[X+\frac{(1-q) Y}{1-q t}\right],
\end{aligned}
$$


where we have used $P_{\lambda}^{(q, t)}[\tau Z]=\tau^{|\lambda|} P_{\lambda}^{(q, t)}[Z]$ ( $\tau$ stands for any monomial in $q$ and $t$ ) and the usual duality [21]

$$
\omega P_{\lambda}^{(q, t)}\left[\frac{1-q}{1-t} Z\right]=b_{\lambda^{\prime}}(t, q) P_{\lambda^{\prime}}^{(t, q)}[Z] .
$$

Using the relation $b_{\lambda}(q, t)=(t / q)^{|\lambda|} b_{\lambda}\left(q^{-1}, t^{-1}\right)$, we then obtain

$\omega_{q, t}^{B} P_{\lambda, \mu}(x, y ; q, t)=t^{|\mu|} b_{\mu^{\prime}}\left(t^{-1},(q t)^{-1}\right) b_{\lambda^{\prime}}\left((q t)^{-1}, q^{-1}\right) P_{\mu^{\prime}, \lambda^{\prime}}\left(x, y ; t^{-1}, q^{-1}\right)$,

which completes the proof.

The scalar product (1.7) does not behave well in the limits $q=t=0$ and $q=$ $t=\infty$. An interesting consequence of Proposition 8 is that it can be used to relate the corresponding specializations of the double Macdonald polynomials. In effect, from the definition of $\omega_{q, t}^{B}$, we have that (3.18) is equivalent to

$$
\left\langle\left\langle\omega_{1,1}^{B} P_{\mu^{\prime}, \lambda^{\prime}}(x, y ; q, t), P_{\nu, \omega}\left(x, y ; t^{-1}, q^{-1}\right)\right\rangle_{1,1}=\delta_{\lambda \nu} \delta_{\mu \omega} .\right.
$$

The limit $q=t=\infty$ of this result is then well defined and reads

$$
\left\langle\left\langle\omega_{1,1}^{B} s_{\mu^{\prime}}[X+Y] s_{\lambda^{\prime}}(y), s_{\nu}(x) s_{\omega}(y)\right\rangle\right\rangle_{1,1}=\delta_{\lambda \nu} \delta_{\mu \omega} .
$$

The following identity involving products of four Littlewood-Richardson coefficients (which to the best of our knowledge is not in the literature) follows from the previous duality.

Proposition 9 For any partitions $\lambda, \mu$, v, and $\omega$, we have

$$
\sum_{\gamma, \eta, \sigma, \tau}(-1)^{|\tau|} c_{\tau^{\prime} \nu}^{\gamma} c_{\gamma \eta}^{\lambda} c_{\eta \mu}^{\sigma} c_{\sigma \tau}^{\omega}=\delta_{\lambda \nu} \delta_{\mu \omega},
$$

where $\gamma, \eta, \sigma, \tau$ run over all partitions and where $c_{\mu \nu}^{\lambda}$ is the corresponding LittlewoodRichardson coefficient. Equivalently, if as in [8], we let

$$
c_{\lambda \mu \eta}^{\nu}=\sum_{\xi} c_{\lambda \mu}^{\xi} c_{\xi \eta}^{\nu}
$$

the identity reads

$$
\sum_{\eta, \tau}(-1)^{|\tau|} c_{\tau^{\prime} \nu \eta}^{\lambda} c_{\eta \mu \tau}^{\omega}=\delta_{\lambda \nu} \delta_{\mu \omega}
$$

Proof The scalar product (1.7) in the limit $q=t=1$ is such that

$$
\left\langle\left\langle s_{\mu}[X] s_{\lambda}[X+Y], s_{\gamma}[X] s_{\nu}[X+Y]\right\rangle\right\rangle_{1,1}=\delta_{\mu \gamma} \delta_{\lambda \nu} .
$$


Moreover, the action of $\omega_{1,1}^{B}$ on $s_{\mu}[X] s_{\lambda}[X+Y]$ is easily seen to be

$$
\omega_{1,1}^{B} s_{\mu}[X] s_{\lambda}[X+Y]=(-1)^{|\mu|} s_{\mu}[X] s_{\lambda^{\prime}}[X+Y] .
$$

It will thus prove convenient to expand $s_{\mu^{\prime}}[X+Y] s_{\lambda^{\prime}}(y)$ and $s_{\nu}(x) s_{\omega}(y)$ in the $s_{\mu}[X] s_{\lambda}[X+Y]$ basis. We have

$$
\begin{aligned}
s_{\lambda^{\prime}}[Y] & =s_{\lambda^{\prime}}[-X+X+Y]=\sum_{\gamma, \eta} c_{\gamma \eta}^{\lambda^{\prime}} s_{\gamma}[-X] s_{\eta}[X+Y] \\
& =\sum_{\gamma, \eta} c_{\gamma \eta}^{\lambda^{\prime}}(-1)^{|\gamma|} s_{\gamma^{\prime}}[X] s_{\eta}[X+Y] .
\end{aligned}
$$

Hence,

$$
\begin{aligned}
\omega_{1,1}^{B} s_{\mu^{\prime}}[X+Y] s_{\lambda^{\prime}}[Y] & =\omega_{1,1}^{B} \sum_{\gamma, \eta, \sigma}(-1)^{|\gamma|} c_{\gamma \eta}^{\lambda^{\prime}} c_{\eta \mu^{\prime}}^{\sigma} s_{\gamma^{\prime}}[X] s_{\sigma}[X+Y] \\
& =\sum_{\gamma, \eta, \sigma} c_{\gamma \eta}^{\lambda^{\prime}} c_{\eta \mu^{\prime}}^{\sigma} s_{\gamma^{\prime}}[X] s_{\sigma^{\prime}}[X+Y] .
\end{aligned}
$$

Similarly, after some straightforward computations (using again (3.22)), we obtain

$$
s_{\nu}[X] s_{\omega}[Y]=\sum_{\tau, \pi, \xi}(-1)^{|\tau|} c_{\nu \tau^{\prime}}^{\xi} c_{\pi \tau}^{\omega} s_{\xi}[X] s_{\pi}[X+Y]
$$

Finally, replacing the expansions (3.23) and (3.24) in (3.19), we get thanks to (3.21) the identity

$$
\sum_{\gamma, \eta, \sigma, \tau}(-1)^{|\tau|} c_{\gamma \eta}^{\lambda^{\prime}} c_{\eta \mu^{\prime}}^{\sigma} c_{\nu \tau^{\prime}}^{\gamma^{\prime}} c_{\sigma^{\prime} \tau}^{\omega}=\delta_{\lambda \nu} \delta_{\mu \omega} .
$$

The proposition then follows since

$$
\begin{aligned}
\sum_{\gamma, \eta, \sigma, \tau}(-1)^{|\tau|} c_{\gamma \eta}^{\lambda^{\prime}} c_{\eta \mu^{\prime}}^{\sigma} c_{\nu \tau^{\prime}}^{\gamma^{\prime}} c_{\sigma^{\prime} \tau}^{\omega} & =\sum_{\gamma, \eta, \sigma, \tau}(-1)^{|\tau|} c_{\gamma^{\prime} \eta^{\prime}}^{\lambda} c_{\eta^{\prime} \mu}^{\sigma^{\prime}} c_{\nu \tau^{\prime}}^{\gamma^{\prime}} c_{\sigma^{\prime} \tau}^{\omega} \\
& =\sum_{\gamma, \eta, \sigma, \tau}(-1)^{|\tau|} c_{\tau^{\prime} \nu}^{\gamma} c_{\gamma \eta}^{\lambda} c_{\eta \mu}^{\sigma} c_{\sigma \tau}^{\omega},
\end{aligned}
$$

where we used $c_{\mu \nu}^{\lambda}=c_{\mu^{\prime} v^{\prime}}^{\lambda^{\prime}}$ and $c_{\mu \nu}^{\lambda}=c_{\nu \mu}^{\lambda}$.

\subsection{Evaluation}

We now provide an explicit formula for the evaluation of the double Macdonald polynomials. The first point to clarify is the way we could specialize the variables $x$ and $y$. 
For this, we recall that the most general evaluation of the usual Macdonald polynomial is [21, eqs VI (6.16)-(6.17)]

$$
P_{\lambda}^{(q, t)}\left[\frac{1-u}{1-t}\right]=\prod_{s \in \lambda} \frac{t^{l^{\prime}(s)}-q^{a^{\prime}(s)} u}{1-q^{a(s)} t^{l(s)+1}}=: w_{\lambda}(u ; q, t),
$$

where the plethysm is such that:

$$
p_{r}\left[\frac{1-u}{1-t}\right]=\frac{1-u^{r}}{1-t^{r}}
$$

Considering the factorized expression (1.10), one sees that in order to evaluate the first term $P_{\lambda}^{(q, q t)}\left[X+\frac{q(1-t)}{1-q t} Y\right]$, we need to bring the argument in the proper form, that is, set $X$ and $Y$ such that

$$
X+\frac{q(1-t)}{1-q t} Y=\frac{1-u}{1-q t}
$$

This clearly requires $X$ and $Y$ to be of the form

$$
X=q^{a}\left(1+q t+\cdots+(q t)^{m}\right) \quad \text { and } \quad Y=t^{b}\left(1+t+\cdots+t^{N-m}\right),
$$

and the condition (3.26) further imposes $a=1-m$ and $b=m$ (so that the resulting $u$ is $q^{m} t^{N}$ ). We thus define the evaluation as

$$
\mathrm{E}_{N, m}\left(P_{\lambda, \mu}(x, y ; q, t)\right)=\left.P_{\lambda, \mu}(x, y ; q, t)\right|_{x_{i}=\frac{t^{i-1}}{q^{m-i}}, y_{i}=t^{m+i-1}} .
$$

Corollary 10 With the evaluation defined in (3.27) and $w_{\lambda}(u ; q, t)$ defined in (3.25), we have

$$
E_{N, m}\left(P_{\lambda, \mu}(x, y ; q, t)\right)=\frac{t^{m|\mu|}}{q^{(m-1)|\lambda|}} w_{\lambda}\left(q^{m} t^{N} ; q, q t\right) w_{\mu}\left(t^{N-m} ; q t, t\right)
$$

Proof The specialization gives

$$
X=x_{1}+x_{2}+\cdots+x_{m}=\frac{1}{q^{m-1}}+\frac{t}{q^{m-2}}+\cdots+t^{m-1}=q^{1-m} \frac{1-(q t)^{m}}{1-q t}
$$

and

$$
Y=y_{1}+\cdots+y_{N-m}=t^{m}+\cdots+t^{N-1}=t^{m} \frac{1-t^{N-m}}{1-t} .
$$


The evaluation of the two terms in the factorization (1.10) is immediate

$$
\begin{aligned}
\mathrm{E}_{N, m}\left(P_{\lambda}^{(q, q t)}\left[X+\frac{q(1-t)}{1-q t} Y\right]\right) & =\frac{1}{q^{(m-1)|\lambda|}} P_{\lambda}^{(q, q t)}\left[\frac{1-q^{m} t^{N}}{1-q t}\right] \\
& =\frac{1}{q^{(m-1)|\lambda|}} w_{\lambda}\left(q^{m} t^{N} ; q, q t\right)
\end{aligned}
$$

and

$$
\begin{aligned}
\mathrm{E}_{N, m}\left(P_{\mu}^{(q t, t)}[Y]\right) & =P_{\mu}^{(q t, t)}\left[t^{m} \frac{1-t^{N-m}}{1-t}\right]=t^{m|\mu|} P_{\mu}^{(q t, t)}\left[\frac{1-t^{N-m}}{1-t}\right] \\
& =t^{m|\mu|} w_{\mu}\left(t^{N-m} ; q t, t\right),
\end{aligned}
$$

whose product yields the announced result.

\subsection{Kostka coefficients}

Recall that the integral form of the Macdonald polynomials is [21, VI.8.3]

$$
J_{\lambda}(x ; q, t)=c_{\lambda}(q, t) P_{\lambda}(x ; q, t),
$$

where

$$
c_{\lambda}(q, t)=\prod_{s \in \lambda}\left(1-q^{a(s)} t^{l(s)+1}\right) .
$$

We define the integral form of the double Macdonald polynomials to be

$$
J_{\lambda, \mu}(x, y ; q, t)=c_{\lambda}(q, q t) c_{\mu}(q t, t) P_{\lambda, \mu}(x, y ; q, t) .
$$

Recall also the definition of the modified Macdonald polynomials

$$
H_{\lambda}(x ; q, t)=J_{\lambda}^{(q, t)}\left[\frac{X}{1-t}\right]
$$

which, when expanded in the Schur basis, are such that (this is equivalent to [21, VI.8.11])

$$
H_{\lambda}(x ; q, t)=\sum_{\mu} K_{\mu \lambda}(q, t) s_{\mu}(x)
$$

where $K_{\mu \lambda}(q, t)$ is the $q, t$-Kostka coefficient. It has been shown in [18] that $K_{\mu \lambda}(q, t) \in \mathbb{N}[q, t]$. Recall also that $K_{\mu \lambda}(1,1)=\chi_{\left(1^{n}\right)}^{\mu}$, where $\chi_{\left(1^{n}\right)}^{\mu}$ is the value of the irreducible $S_{n}$-character $\chi^{\mu}$ at the class of the identity [21, VI.8.16]. Equivalently, $K_{\mu \lambda}(1,1)$ corresponds to the number of standard tableaux of shape $\mu$ (cf. [3, eqs (2.1) and (9.7)]). 
By analogy, we define

$$
H_{\lambda, \mu}(x, y ; q, t)=\varphi\left(J_{\lambda, \mu}(x, y ; q, t)\right)
$$

where $\varphi$ is the the homomorphism whose action on the power sums is

$$
p_{n}[X] \mapsto p_{n}[X] \quad \text { and } \quad p_{n}[X+Y] \mapsto \frac{1}{1-t^{n}} p_{n}[X+Y]
$$

Note that the homomorphism $\varphi$ is equivalent to the plethystic substitution

$$
X \mapsto X \quad \text { and } \quad X+Y \mapsto \frac{1}{(1-t)}(X+Y)
$$

From Theorem 5, after some straightforward manipulations, we obtain that

$$
\begin{aligned}
H_{\lambda, \mu}(x, y ; q, t) & =J_{\lambda}^{(q, q t)}\left[\frac{X}{1-q t}+\frac{q Y}{1-q t}\right] J_{\mu}^{(q t, t)}\left[\frac{Y}{1-t}+\frac{t X}{1-t}\right] \\
& =H_{\lambda}^{(q, q t)}[X+q Y] H_{\mu}^{(q t, t)}[t X+Y] .
\end{aligned}
$$

Our interest is to introduce $B_{n}$-analogs of the Kostka coefficients by expanding $H_{\lambda, \mu}(x, y ; q, t)$ in terms of the Schur functions associated to the irreducible characters of $B_{n}$ (see [21, p. 178]):

$$
s_{\lambda, \mu}(x, y)=s_{\lambda}(x) s_{\mu}(y) .
$$

Observe that from Theorem 5, these Schur functions correspond to the specialization $q=t=0$ of $P_{\lambda, \mu}(x ; q, t)$, namely $s_{\lambda, \mu}(x, y)=P_{\lambda, \mu}(x, y ; 0,0)$ (see also [6,7]).

Now define the double Kostka coefficients $K_{\kappa, \gamma} \lambda, \mu(q, t)$ through the expansion

$$
H_{\lambda, \mu}(x, y ; q, t)=\sum_{\kappa, \gamma} K_{\kappa, \gamma} \lambda, \mu(q, t) s_{\kappa, \gamma}(x, y) .
$$

The reader is referred to Appendix D for tables of double Kostka coefficients up to degree $n=3$. We first connect $K_{\kappa, \gamma} \lambda, \mu(1,1)$ to the representation theory of the hyperoctahedral group $B_{n}$.

Proposition 11 Let $\lambda$ and $\mu$ be such that $|\lambda|+|\mu|=n$. Then, $K_{\kappa, \gamma} \lambda, \mu(1,1)$ is the dimension of the irreducible representation of $B_{n}$ indexed by the pairs of partitions $\kappa, \gamma$. In particular, $K_{\kappa, \gamma} \lambda, \mu(1,1)$ does not depend on $\lambda$ and $\mu$.

Proof When $q=t=1$, it is known [3, eq. (9.6)] that

$$
H_{\lambda}(x ; 1,1)=p_{1}^{|\lambda|} .
$$


It thus follows from (3.32) that

$$
H_{\lambda, \mu}(x, y ; 1,1)=H_{\lambda}^{(1,1)}[X+Y] H_{\mu}^{(1,1)}[X+Y]=\left(p_{1}[X+Y]\right)^{|\lambda|+|\mu|}=p_{1^{n}}[X+Y] .
$$

But it is known (see [21, p. 178]) that

$$
p_{\rho}[X+Y] p_{\sigma}[X-Y]=\sum_{\kappa, \gamma} \chi_{\rho, \sigma}^{\kappa, \gamma} s_{\kappa, \gamma}(x, y)
$$

where $\chi_{\rho, \sigma}^{\kappa, \gamma}$ stands for the $B_{n}$-character indexed by the irreducible representation $\kappa, \gamma$ at the class indexed by $\rho, \sigma$. If we set $\rho=1^{n}$ and $\sigma=\emptyset$, then we obtain that

$$
H_{\lambda, \mu}(x, y ; 1,1)=p_{1^{n}}[X+Y]=\sum_{\kappa, \gamma} \chi_{1^{n}, \emptyset}^{\kappa, \gamma} s_{\kappa, \gamma}(x, y)
$$

which proves the proposition since the class indexed by $1^{n}, \emptyset$ is the class of the identity, in which case the character yields the dimension of the representation.

Proposition 11 implies that $K_{\kappa, \gamma} \lambda, \mu(1,1)$ is the number of pairs of standard Young tableaux of respective shapes $\kappa$ and $\gamma$ filled (without repetitions) with the numbers $\{1,2,3, \ldots, n\}$, where $n=|\kappa|+|\gamma|$. For instance, $K_{(2,1),(1)} \lambda, \mu(1,1)=8$ is the number of pairs of standard Young tableaux of shape $(2,1),(1)$ :
\begin{tabular}{|l|l|}
\hline 1 & 2 \\
\hline 3 &
\end{tabular}

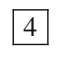
\begin{tabular}{|l|l|}
\hline 1 & 3 \\
\hline 2 &
\end{tabular}
4
\begin{tabular}{|l|l|}
\hline 1 & 2 \\
\hline 4 &
\end{tabular}
3
\begin{tabular}{|l|l|}
\hline 1 & 4 \\
\hline 2 &
\end{tabular}
3
\begin{tabular}{|l|l|}
\hline 1 & 3 \\
\hline 4 &
\end{tabular}
\begin{tabular}{|l|l|l|}
\hline 2 & 4 \\
\hline 3 & \\
\hline
\end{tabular}
2
\begin{tabular}{|l|l|}
\hline 2 & 3 \\
\hline 4
\end{tabular}
\begin{tabular}{|l|l|}
\hline 2 & 4 \\
\hline 3 &
\end{tabular},
1 .

We now show the positivity of the coefficients $K_{\kappa, \gamma} \lambda, \mu(q, t)$. The proposition implies that the double Kostkas (of $B_{n}$-type) can be obtained from the usual $q, t$-Kostka (of $S_{n}$-type). Therefore, from the combinatorial point of view, this new hyperoctahedral perspective does not shed any light on the usual $q$, $t$-Kostka. In Sect. 5, we will discuss how an interesting new combinatorics appears in the non-stable sector that interpolates between the $S_{n}$ and $B_{n}$ cases.

Proposition 12 We have

$$
K_{\kappa, \gamma \lambda, \mu}(q, t)=\sum_{\nu, \omega, \alpha, \beta, \rho, \sigma} K_{\nu \lambda}(q, q t) K_{\omega \mu}(q t, t) c_{\alpha \beta}^{\nu} c_{\rho \sigma}^{\omega} c_{\alpha \rho}^{\kappa} c_{\beta \sigma}^{\gamma} q^{|\beta|} t^{|\rho|}
$$

where $c_{\alpha \beta}^{\lambda}$ is the corresponding Littlewood-Richardson coefficient. In particular, $K_{\kappa, \gamma \lambda, \mu}(q, t) \in \mathbb{N}[q, t]$. 
Proof From (3.30) and (3.32), we have

$$
\begin{aligned}
& H_{\lambda, \mu}(x, y ; q, t)=\sum_{\nu, \omega} K_{\nu \lambda}(q, q t) K_{\omega \mu}(q t, t) s_{\nu}[X+q Y] s_{\omega}[t X+Y] \\
& =\sum_{\nu, \omega, \alpha, \beta, \rho, \sigma} K_{\nu \lambda}(q, q t) K_{\omega \mu}(q t, t) c_{\alpha \beta}^{\nu} c_{\rho \sigma}^{\omega} s_{\alpha}[X] s_{\beta}[q Y] s_{\rho}[t X] s_{\sigma}[Y] \\
& =\sum_{\nu, \omega, \alpha, \beta, \rho, \sigma} K_{\nu \lambda}(q, q t) K_{\omega \mu}(q t, t) c_{\alpha \beta}^{\nu} c_{\rho \sigma}^{\omega} q^{|\beta|} t^{|\rho|} s_{\alpha}[X] s_{\rho}[X] s_{\beta}[Y] s_{\sigma}[Y] \\
& =\sum_{\nu, \omega, \alpha, \beta, \rho, \sigma, \kappa, \gamma} K_{\nu \lambda}(q, q t) K_{\omega \mu}(q t, t) c_{\alpha \beta}^{\nu} c_{\rho \sigma}^{\omega} c_{\alpha \rho}^{\kappa} c_{\beta \sigma}^{\gamma} q^{|\beta|} t^{|\rho|} s_{\kappa}[X] s_{\gamma}[Y] \\
& =\sum_{\kappa, \gamma}\left(\sum_{\nu, \omega, \alpha, \beta, \rho, \sigma} K_{\nu \lambda}(q, q t) K_{\omega \mu}(q t, t) c_{\alpha \beta}^{\nu} c_{\rho \sigma}^{\omega} c_{\alpha \rho}^{\kappa} c_{\beta \sigma}^{\gamma} q^{|\beta|} t^{|\rho|}\right) s_{\kappa, \gamma}(x, y) .
\end{aligned}
$$

This proves the first assertion. The positivity of $K_{\kappa, \gamma} \lambda, \mu(q, t)$ then follows from the positivity of the $q, t$-Kostkas [18] and the Littlewood-Richardson coefficients.

Remark 13 Propositions 11 and 12 suggest that there exists a bigraded module of the regular representation of the hyperoctahedral group $B_{n}$ whose Frobenius series corresponds to the Schur expansion of $H_{\lambda, \mu}(x, y ; q, t)$. This point will be discussed further in Remark 22.

Corollary 14 The double Kostka coefficients have the following symmetries:

$$
\begin{aligned}
& K_{\kappa, \gamma \lambda, \mu}(q, t)=q^{\bar{n}\left(\mu^{\prime}, \lambda^{\prime}\right)} t^{\bar{n}(\lambda, \mu)} K_{\gamma^{\prime}, \kappa^{\prime} \lambda, \mu}\left(q^{-1}, t^{-1}\right) \quad \text { and } \\
& K_{\kappa, \gamma \lambda, \mu}(q, t)=K_{\gamma^{\prime}, \kappa^{\prime} \mu^{\prime}, \lambda^{\prime}}(t, q),
\end{aligned}
$$

where $\bar{n}(\lambda, \mu)=n(\lambda)+|\mu|+n\left(\mu^{\prime}\right)+n(\mu)$.

Proof For the usual Kostka coefficients, the analogous form of the first symmetry is equivalent to (see e.g., [3, eq. (9.9)])

$$
H_{\lambda}(x ; q, t)=q^{n\left(\lambda^{\prime}\right)} t^{n(\lambda)} \omega H_{\lambda}\left(x ; q^{-1}, t^{-1}\right),
$$

where $\omega$ is such that $\omega s_{\mu}=s_{\mu^{\prime}}$. If we extend $\omega$ to the space of bisymmetric functions as $\omega s_{\mu}[X]=s_{\mu^{\prime}}[X]$ and $\omega s_{\mu}[Y]=s_{\mu^{\prime}}[Y]$ (which implies for instance that $\omega s_{\lambda}[X+$ $\left.q Y]=s_{\lambda^{\prime}}[X+q Y]^{6}\right)$, then we obtain from (3.32)

6 This is seen from

$$
\begin{aligned}
\omega s_{\lambda}[X+q Y] & =\omega \sum_{\mu, v} c_{\mu \nu}^{\lambda} s_{\mu}[X] q^{|v|} s_{\nu}[Y]=\sum_{\mu, v} c_{\mu \nu}^{\lambda} s_{\mu^{\prime}}[X] q^{|v|} s_{v^{\prime}}[Y] \\
& =\sum_{\mu, v} c_{\mu^{\prime} \nu^{\prime}}^{\lambda^{\prime}} s_{\mu^{\prime}}[X] s_{\nu^{\prime}}[q Y]=s_{\lambda^{\prime}}[X+q Y] .
\end{aligned}
$$




$$
\begin{aligned}
H_{\lambda, \mu}(x, y ; q, t)= & q^{n\left(\lambda^{\prime}\right)}(q t)^{n(\lambda)} \omega\left(H_{\lambda}^{\left(q^{-1},(q t)^{-1}\right)}[X+q Y]\right)(q t)^{n\left(\mu^{\prime}\right)} t^{n(\mu)} \\
& \omega\left(H_{\mu}^{\left((q t)^{-1}, t^{-1}\right)}[t X+Y]\right) \\
= & q^{n\left(\lambda^{\prime}\right)}(q t)^{n(\lambda)} q^{|\lambda|} \omega\left(H_{\lambda}^{\left(q^{-1},(q t)^{-1}\right)}\left[q^{-1} X+Y\right]\right)(q t)^{n\left(\mu^{\prime}\right)} t^{n(\mu)} t^{|\mu|} \\
& \omega\left(H_{\mu}^{\left((q)^{-1}, t^{-1}\right)}\left[X+t^{-1} Y\right]\right) \\
= & q^{\bar{n}\left(\mu^{\prime}, \lambda^{\prime}\right)} t^{\bar{n}(\lambda, \mu)} \omega\left(H_{\lambda}^{\left(q^{-1},(q t)^{-1}\right)}\left[q^{-1} X+Y\right]\right) \\
& \omega\left(H_{\mu}^{\left((q t)^{-1}, t^{-1}\right)}\left[X+t^{-1} Y\right]\right) \\
= & q^{\bar{n}\left(\mu^{\prime}, \lambda^{\prime}\right)} t^{\bar{n}(\lambda, \mu)} \omega H_{\lambda, \mu}\left(y, x ; q^{-1}, t^{-1}\right) .
\end{aligned}
$$

But this amounts to

$$
H_{\lambda, \mu}(x, y ; q, t)=q^{\bar{n}\left(\mu^{\prime}, \lambda^{\prime}\right)} t^{\bar{n}(\lambda, \mu)} \omega_{B} H_{\lambda, \mu}\left(x, y ; q^{-1}, t^{-1}\right),
$$

where $\omega_{B} s_{\lambda, \mu}(x, y)=s_{\mu^{\prime}, \lambda^{\prime}}(x, y)$, which implies the first relation.

For the second relation, we use the other Macdonald symmetry [3, eq. (9.8)]

$$
H_{\lambda}(x ; q, t)=\omega H_{\lambda^{\prime}}(x ; t, q),
$$

to obtain

$$
H_{\lambda, \mu}(x, y ; q, t)=\omega\left(H_{\lambda^{\prime}}^{(q t, q)}[X+q Y]\right) \omega\left(H_{\mu^{\prime}}^{(t, q t)}[t X+Y]\right)=\omega H_{\mu^{\prime}, \lambda^{\prime}}(y, x ; t, q) .
$$

But this is equivalent to

$$
H_{\lambda, \mu}(x, y ; q, t)=\omega_{B} H_{\mu^{\prime}, \lambda^{\prime}}(x, y ; t, q),
$$

and the result follows.

It is known that for $|\lambda|=n[19$, Prop. 3.5.20] or [21, ex. 2 p. 362],

$$
K_{(n) \lambda}(q, t)=t^{n(\lambda)} \quad \text { and } \quad K_{\left(1^{n}\right) \lambda}(q, t)=q^{n\left(\lambda^{\prime}\right)}
$$

These correspond to the two inequivalent representations of dimension 1 of $S_{n}$. In the case of $B_{n}$, there are 4 inequivalent representations of dimension 1 . The corresponding double Kostkas, which will prove important in the next section, are given in the next Corollary. 
Corollary 15 Let $|\lambda|+|\mu|=n$. We have

$$
K_{(n), \emptyset \lambda, \mu}(q, t)=q^{n(\lambda)} t^{|\mu|+n(\lambda)+n(\mu)} \quad \text { and } \quad K_{\emptyset,(n) \lambda, \mu}(q, t)=q^{|\lambda|+n(\lambda)} t^{n(\lambda)+n(\mu)} .
$$

By symmetry, i.e., using the first relation of (3.35), we also have

$$
\begin{aligned}
& K_{\emptyset,\left(1^{n}\right) \lambda, \mu}(q, t)=q^{|\lambda|+n\left(\lambda^{\prime}\right)+n\left(\mu^{\prime}\right)} t^{n\left(\mu^{\prime}\right)} \text { and } \\
& K_{\left(1^{n}\right), \emptyset \lambda, \mu}(q, t)=q^{n\left(\lambda^{\prime}\right)+n\left(\mu^{\prime}\right)} t^{|\mu|+n\left(\mu^{\prime}\right)} .
\end{aligned}
$$

Proof We will only prove the expression for $K_{\emptyset,(n)} \lambda, \mu(q, t)$ since that of $K_{(n), \emptyset} \lambda, \mu(q, t)$ can be obtained in a similar way. From $(3.32), K_{\emptyset,(n)} \lambda, \mu(q, t)$ is equal to the coefficient of $s_{\emptyset,(n)}(x, y)=s_{(n)}[Y]$ in $H_{\lambda, \mu}(x, y ; q, t)$. In the product form (3.32), we can thus set $X=0$ and search for the coefficient of $s_{(n)}[Y]$ in $H_{\lambda}^{(q, q t)}[q Y] H_{\mu}^{(q t, t)}[Y]=q^{|\lambda|} H_{\lambda}^{(q, q t)}[Y] H_{\mu}^{(q t, t)}[Y]$. Let us first expand the latter product:

$$
\begin{aligned}
H_{\lambda}^{(q, q t)}[Y] H_{\mu}^{(q t, t)}[Y] & =\sum_{\eta, \rho} K_{\eta \lambda}(q, q t) K_{\rho \mu}(q t, t) s_{\eta}[Y] s_{\rho}[Y] \\
& =\sum_{\eta, \rho, \omega} K_{\eta \lambda}(q, q t) K_{\rho \mu}(q t, t) c_{\eta \rho}^{\omega} s_{\omega}[Y] .
\end{aligned}
$$

For $\omega=(n)$, it follows that the non-vanishing Littlewood-Richardson coefficients $c_{\eta \rho}^{(n)}$ are equal to $\delta_{\eta,\left(n_{1}\right)} \delta_{\rho,\left(n_{2}\right)}$ for non-negative integers $n_{1}$ and $n_{2}$ satisfying $n_{1}+n_{2}=n$. The homogeneity of the Kostka coefficients then imposes $n_{1}=|\lambda|$ and $n_{2}=|\mu|$. The desired coefficient is thus equal to $q^{|\lambda|} K_{\left(n_{1}\right) \lambda}(q, q t) K_{\left(n_{2}\right) \mu}(q t, t)$. Using (3.37), the result follows.

\section{The Nabla operator}

\subsection{Review of the usual case: Nabla operator and Frobenius series}

A useful technique to demonstrate that a given symmetric polynomial is Schur positive is to link this expansion to the decomposition of a representation into irreducible ones. By means of the characteristic map, a symmetric polynomial is transformed into a class function of $S_{n}$. Under this map, a Schur function is associated to an irreducible character of $S_{n}$. It follows that a symmetric function $F$ associated to a representation is Schur positive: this symmetric function can be expanded as $F=$ $\sum c_{\lambda} s_{\lambda}$, where by construction, $c_{\lambda}$ is a non-negative integer since it corresponds to the multiplicity of the irreducible representation $\lambda$ in the larger representation under consideration.

Let

$$
\tilde{H}_{\lambda}(x ; q, t)=t^{n(\lambda)} H_{\lambda}\left(x ; q, t^{-1}\right)=\sum_{\mu} \tilde{K}_{\mu \lambda}(q, t) s_{\mu}(x),
$$


(hence, $\left.\tilde{K}_{\mu \lambda}(q, t)=t^{n(\lambda)} K_{\mu \lambda}\left(q, t^{-1}\right)\right)$. Garsia and Haiman [14] were able to construct bigraded $S_{n}$-modules $\mathcal{H}_{\mu}$ (the so-called Garsia-Haiman modules) such that ${ }^{7}$

$$
\operatorname{Frob}_{q, t}\left(\mathcal{H}_{\mu}\right)=\tilde{H}_{\mu}(x ; q, t)
$$

(More precisely, this result is conjectured in [14] and proved in [18].) The GarsiaHaiman modules belong to the larger module $\mathcal{D}_{n}$ of diagonal harmonics whose dimension is $(n+1)^{n-1}$. Quite remarkably, the bigraded Hilbert series of $\mathcal{D}_{n}$ can be calculated from the the operator $\nabla$ introduced by Bergeron and Garsia [4] (see also [5] and [3], beginning of Sect. 9.6) and defined as follows:

$$
\nabla \tilde{H}_{\lambda}=q^{n\left(\lambda^{\prime}\right)} t^{n(\lambda)} \tilde{H}_{\lambda}
$$

In other words, $\nabla$ is defined from its action on $\tilde{H}_{\lambda}$ and because these polynomials form a basis, this provides its action on any symmetric function. It has been proven (and this is a highly non-trivial result) that the action of $\nabla$ on $e_{n}$, when expanded in the Schur basis,

$$
\nabla e_{n}=\sum_{\lambda} c_{\lambda}(q, t) s_{\lambda}
$$

can be interpreted as the bigraded Frobenius series of $\mathcal{D}_{n}$ (see e.g., [19, Theorem 4.2.5] and Sects. 3.5, 4.1 and 4.2 therein for the subsequent results of this section). Moreover, in the case $q=1 / t$, it has been shown that

$$
\left\langle\nabla_{q=1 / t} e_{n}, p_{1}^{n}\right\rangle=\left(\frac{[n+1]_{t}}{t^{n / 2}}\right)^{n-1},
$$

where

$$
[k]_{t}=\frac{\left(1-t^{k}\right)}{(1-t)}=\left(1+t+\cdots+t^{k-1}\right) .
$$

Specializing this result to $t=1$, one recovers $(n+1)^{n-1}$ as the dimension of $\mathcal{D}_{n}$. Note that for $q$ and $t$ generic, there is no factorization of the type

$$
\left\langle\nabla e_{n}, p_{1}^{n}\right\rangle=(f(q, t))^{n-1}
$$

\footnotetext{
7 Recall that the (bigraded) Frobenius series of a bigraded module $W=\bigoplus_{i, j} W^{i, j}$ is written as [16]$$
\operatorname{Frob}_{q, t}(W)=\sum_{i, j} t^{i} q^{j} \sum_{\lambda} s_{\lambda} M\left(\chi^{\lambda}, W^{i, j}\right),
$$

where $M\left(\chi^{\lambda}, W^{i, j}\right)$ is the multiplicity of the irreducible character $\chi^{\lambda}$ in the module $W^{i, j}$. In other words, given a decomposition of $W^{i, j}$ into irreducible submodules, $M\left(\chi^{\lambda}, W^{i, j}\right)$ is the number of such submodules that have character $\chi^{\lambda}$.
} 
for some polynomial $f(q, t) \in \mathbb{N}[q, t]$. The coefficient of $e_{n}$ in $\nabla e_{n}$,

$$
C_{n}(q, t)=\left\langle\nabla e_{n}, e_{n}\right\rangle,
$$

is also of particular interest. It corresponds to the bigraded Hilbert series of the subspace $\mathcal{A}_{n}$ of alternants in $\mathcal{D}_{n}$. There is a combinatorial interpretation for $C_{n}(q, t)$ (reviewed in [16, Chapter 3]), but there is no known closed-form expression. However, when $q=1 / t, C_{n}(q, t)$ reduces to

$$
C_{n}\left(t^{-1}, t\right)=t^{-\left(\begin{array}{c}
n \\
2
\end{array}\right)} \frac{1}{[n+1]_{t}}\left[\begin{array}{c}
2 n \\
n
\end{array}\right]_{t},
$$

which is a $t$-analog of the Catalan number

$$
C_{n}=\frac{1}{n+1}\left(\begin{array}{c}
2 n \\
n
\end{array}\right) \text {. }
$$

The dimension of the subspace of alternants $\mathcal{A}_{n}$ is thus given by the $n$-th Catalan number.

\subsection{Heuristic strategy for the Nabla operator in the hyperoctahedral case}

Proposition 11 provides a connection between the hyperoctahedral group and the double Macdonald polynomials. In this subsection, we will deepen this connection by the introduction of Nabla operators that will produce another $B_{n}$ datum, namely the $B_{n}$ version of the dimension formula $(n+1)^{n-1}$.

Recall that the group $S_{n}$ is a Coxeter group of type $A$, namely $A_{n-1}$. The formula $(n+1)^{n-1}$ turns out to be the $A_{n-1}$ version of the dimension formula $(h+1)^{r}$ [15], where $h$ and $r$ are, respectively, the Coxeter number and the rank of the corresponding Coxeter group ( $h=n$ and $r=n-1$ for $A_{n-1}$ ). Now, the hyperoctahedral group of rank $n$ is equivalent to the group of signed permutations of $n$ objects. Its generators are $\left\{s_{0}, s_{1}, \ldots, s_{n-1}\right\}$, where $s_{0}$ is the sign change, and where the remaining generators are the elementary transpositions that generate $S_{n}$. This description makes clear that the hyperoctahedral group is the Coxeter group $B_{n}$. Given that for $B_{n}$, the Coxeter number and the rank are, respectively, $h=2 n$ and $r=n$, the expected $B_{n}$-form of the dimension is $(2 n+1)^{n}$. This is precisely the value we will obtain.

We first need to formulate the correct definition of the Nabla operators in the $B_{n}$-case. In order to do so, let us briefly reexamine the usual case from a heuristicconstructive point of view. From (3.37), the eigenvalue of $\nabla$ on $\tilde{H}_{\lambda}$ corresponds to $K_{\left(1^{n}\right) \lambda}\left(q, t^{-1}\right) / K_{(n) \lambda}\left(q, t^{-1}\right)$, that is, to the ratio of the Kostka coefficients associated to representations of $S_{n}$ of dimension 1 . This is the guiding observation that we will use to define the $B$ version of the Nabla operator.

As noted before Corollary 15, in our case, there are four pairs of partitions whose Kostka coefficient is equal to 1 when $q=t=1$ : 


$$
K_{(n), \emptyset \lambda, \mu}(1,1)=K_{\left(1^{n}\right), \emptyset \lambda, \mu}(1,1)=K_{\emptyset,(n) \lambda, \mu}(1,1)=K_{\emptyset,\left(1^{n}\right) \lambda, \mu}(1,1)=1 .
$$

There are thus four choices for the rescaling factor that sets equal to 1 a given double Kostka. We will choose to rescale $K_{\left(1^{n}\right), \emptyset}, \mu, \mu(q, t)$ to 1 by redefining $H_{\lambda, \mu}(x, y ; q, t)$ as (this choice has no impact on the definition of a Nabla operator)

$$
\begin{aligned}
\tilde{H}_{\lambda, \mu}(x, y ; q, t) & =\frac{1}{K_{\left(1^{n}\right), \emptyset \lambda, \mu}\left(q, t^{-1}\right)} H_{\lambda, \mu}\left(x, y ; q, t^{-1}\right) \\
& =\sum_{\kappa, \gamma} \tilde{K}_{\kappa, \gamma \lambda, \mu}(q, t) s_{\kappa, \gamma}(x, y) .
\end{aligned}
$$

By (3.38), we have the more explicit relationship between $\tilde{H}_{\lambda, \mu}$ and $H_{\lambda, \mu}$ :

$$
\tilde{H}_{\lambda, \mu}(x, y ; q, t)=q^{-n\left(\lambda^{\prime}\right)-n\left(\mu^{\prime}\right)} t^{|\mu|+n\left(\mu^{\prime}\right)} H_{\lambda, \mu}\left(x, y ; q, t^{-1}\right) .
$$

Observe also that $\tilde{K}_{\kappa, \gamma} \lambda, \mu(q, t)$ now belongs to $\mathbb{N}\left[q^{ \pm 1}, t^{ \pm 1}\right]$.

Following our guiding observation and (4.4), there are two natural and independent ways to define a Nabla operator in the $B$ case, that is, two natural choices of the eigenvalue assigned to $\tilde{H}_{\lambda, \mu}(x, y ; q, t)$. First, we can let the eigenvalue be

$$
\begin{aligned}
\frac{K_{\emptyset,(n) \lambda, \mu}\left(q, t^{-1}\right)}{K_{\left(1^{n}\right), \emptyset \lambda, \mu}\left(q, t^{-1}\right)} & =\frac{q^{|\lambda|+n(\lambda)} t^{-n(\lambda)-n(\mu)}}{q^{n\left(\lambda^{\prime}\right)+n\left(\mu^{\prime}\right)} t^{-|\mu|-n\left(\mu^{\prime}\right)}} \\
& =q^{|\lambda|+n(\lambda)-n\left(\lambda^{\prime}\right)-n\left(\mu^{\prime}\right)} t^{|\mu|+n\left(\mu^{\prime}\right)-n(\lambda)-n(\mu)} .
\end{aligned}
$$

Note that the two pairs of partitions, $(\emptyset,(n))$ and $\left(\left(1^{n}\right), \emptyset\right)$, are conjugate of each other. Hence, the first Nabla operator $\nabla^{B}$ is defined as

$$
\nabla^{B} \tilde{H}_{\lambda, \mu}=q^{|\lambda|+\hat{n}\left(\mu^{\prime}, \lambda^{\prime}\right)} t^{|\mu|+\hat{n}(\lambda, \mu)} \tilde{H}_{\lambda, \mu},
$$

where

$$
\hat{n}(\lambda, \mu)=n\left(\mu^{\prime}\right)-n(\lambda)-n(\mu) .
$$

Alternatively, we can also define the eigenvalue of the Nabla operator to be the ratio:

$$
\begin{aligned}
\frac{K_{\emptyset,\left(1^{n}\right) \lambda, \mu}\left(q, t^{-1}\right)}{K_{(n), \emptyset \lambda, \mu}\left(q, t^{-1}\right)} & =\frac{q^{|\lambda|+n\left(\lambda^{\prime}\right)+n\left(\mu^{\prime}\right)} t^{-n\left(\mu^{\prime}\right)}}{q^{n(\lambda)} t^{-|\mu|-n(\lambda)-n(\mu)}} \\
& =q^{|\lambda|-n(\lambda)+n\left(\lambda^{\prime}\right)+n\left(\mu^{\prime}\right)} t^{|\mu|-n\left(\mu^{\prime}\right)+n(\lambda)+n(\mu)},
\end{aligned}
$$

which leads to

$$
\bar{\nabla}^{B} \tilde{H}_{\lambda, \mu}=q^{|\lambda|-\hat{n}\left(\mu^{\prime}, \lambda^{\prime}\right)} t^{|\mu|-\hat{n}(\lambda, \mu)} \tilde{H}_{\lambda, \mu} .
$$


The operator $\bar{\nabla}^{B}$ does not appear to have generic noteworthy properties. In particular, it is not always Schur positive (up to an overall sign) when acting on the Schur functions $s_{\lambda, \mu}$ (not even when acting on $s_{\emptyset ; 1^{n}}$ ).

From these two commuting operators, one can consider their product $\nabla^{B} \bar{\nabla}^{B}$, whose eigenvalue is readily evaluated from (4.6) and (4.7) to be $q^{2|\lambda|} t^{2|\mu|}$. The factor 2 in both exponents further indicates that the square root of $\nabla^{B} \bar{\nabla}^{B}$ is well defined and thus more fundamental. This operator, defined as

$$
\sqrt{\nabla^{B} \bar{\nabla}^{B}} \tilde{H}_{\lambda, \mu}=q^{|\lambda|} t^{|\mu|} \tilde{H}_{\lambda, \mu}
$$

actually appears to be more interesting. Indeed, its action on a $B$-type Schur displays a Schur-positive expansion (up to an overall sign).

Conjecture 16 The Schur-expansion coefficients of $\left(\sqrt{\nabla^{B} \bar{\nabla}^{B}}\right)^{i} s_{\lambda, \mu}$ belong (up to an overall sign) to $\mathbb{N}\left[q^{ \pm 1}, t^{ \pm 1}\right]$ for every integer $i$ and every pair of partitions $\lambda, \mu$.

In the remainder of the section, we will be solely concerned with the operator $\nabla^{B}$ which connects with $(2 n+1)^{n}$.

\subsection{The explicit action of $\nabla^{B}$ on $s_{\emptyset,(n)}$.}

The analog of $\nabla e_{n} \equiv \nabla s_{\left(1^{n}\right)}$ in the $B_{n}$ case appears to be $\nabla^{B} s_{\emptyset,(n)}$, with $\nabla^{B}$ defined by (4.6). The next proposition gives a simple expression for $\nabla^{B} s \emptyset,(n)$.

Proposition 17 We have

$$
\nabla^{B} s_{\emptyset,(n)}=\frac{1}{(q t)^{\left(\begin{array}{c}
n \\
2
\end{array}\right)}} h_{n}\left[[n]_{q, t} X+[n+1]_{q, t} Y\right]
$$

where

$$
[n]_{q, t}=\frac{q^{n}-t^{n}}{q-t} .
$$

Proof We recall the simple identity (see [21] and e.g. [16, eq. (1.61)])

$$
h_{n}[Z+W]=\sum_{\ell=0}^{n} h_{n-\ell}[Z] h_{\ell}[W]=\sum_{\ell=0}^{n}(-1)^{n-\ell} e_{n-\ell}[-Z] h_{\ell}[W],
$$

where the second equality follows from the duality relation: with $\omega$ such that

$$
\omega p_{r}[X]=(-1)^{r-1} p_{r}[X]=(-1)^{r} p_{r}[-X]
$$

it follows that

$$
p_{\lambda}[-X]=(-1)^{|\lambda|} \omega p_{\lambda}[X] \Longrightarrow h_{\lambda}[-X]=(-1)^{|\lambda|} \omega h_{\lambda}[X]=(-1)^{|\lambda|} e_{\lambda}[X]
$$


Using $h_{\ell}[t Z]=t^{\ell} h_{\ell}[Z]$ (and similar results when $q$ is replaced by $t$ or $h_{\ell}$ is replaced by $e_{\ell}$ ), and suitable choices for $Z$ and $W$, we can write

$$
\begin{aligned}
\sum_{\ell=0}^{n}\left(-\frac{1}{t}\right)^{n-\ell} e_{n-\ell}\left[\frac{X+q Y}{1-q / t}\right] h_{\ell}\left[\frac{X / t+Y}{1-q / t}\right] & =h_{n}\left[\frac{-X / t-q Y / t}{1-q / t}+\frac{X / t+Y}{1-q / t}\right] \\
& =h_{n}[Y] .
\end{aligned}
$$

Since $s_{\emptyset,(n)}(x, y)=s_{(n)}(y)=h_{n}[Y]$, we have thus obtained the following expansion:

$$
s_{\emptyset,(n)}=\sum_{\ell=0}^{n}\left(-\frac{1}{t}\right)^{n-\ell} e_{n-\ell}\left[\frac{X+q Y}{1-q / t}\right] h_{\ell}\left[\frac{X / t+Y}{1-q / t}\right] .
$$

To obtain the action of $\nabla^{B}$ on $s_{\emptyset,(n)}$, it thus suffices to find its action on each term of the sum on the r.h.s. Recall the following expressions (see [21] eq. VI.4.8 and 4.9):

$$
P_{\left(1^{k}\right)}^{(q, t)}[Z]=e_{k}[Z] \quad \text { and } \quad P_{(k)}^{(q, t)}[Z] \propto h_{k}\left[\frac{(1-t)}{(1-q)} Z\right],
$$

where the symbol $\propto$ means that the result holds up to a multiplicative constant. Therefore,

$H_{\left(1^{k}\right)}^{(q, q / t)}[X+q Y] \propto e_{k}\left[\frac{X+q Y}{1-q / t}\right]$ and $H_{(k)}^{(q / t, 1 / t)}[X / t+Y] \propto h_{k}\left[\frac{X / t+Y}{1-q / t}\right]$,

which implies, using (3.32) and (4.5), that

$$
\tilde{H}_{\left(1^{n-\ell}\right),(\ell)}(x, y ; q, t) \propto e_{n-\ell}\left[\frac{X+q Y}{1-q / t}\right] h_{\ell}\left[\frac{X / t+Y}{1-q / t}\right] .
$$

In other words, the product $e_{n-\ell} h_{\ell}$ appearing in the decomposition of $s_{\emptyset ;(n)}$ in (4.10) is proportional to $\tilde{H}_{\left(1^{n-\ell}\right),(\ell)}$, which is itself an eigenfunction of $\nabla^{B}$. Its eigenvalue is read off (4.6) specialized to the case $\lambda=\left(1^{n-\ell}\right)$ and $\mu=(\ell)$ :

$$
\nabla^{B} \tilde{H}_{\left(1^{n-\ell}\right),(\ell)}=\left(\frac{q}{t}\right)^{n(n+1) / 2-n \ell} t^{n} \tilde{H}_{\left(1^{n-\ell}\right),(\ell)} \cdot
$$

After straightforward manipulations, we thus get

$$
\begin{aligned}
\nabla^{B} s_{\emptyset ;(n)} & =\left(\frac{q}{t}\right)^{n(n+1) / 2} \sum_{\ell=0}^{n}\left(\frac{t^{n+1}}{q^{n}}\right)^{\ell}(-1)^{n-\ell} e_{n-\ell}\left[\frac{X+q Y}{1-q / t}\right] h_{\ell}\left[\frac{X / t+Y}{1-q / t}\right] \\
& =\left(\frac{q}{t}\right)^{n(n+1) / 2} \sum_{\ell=0}^{n} h_{n-\ell}\left[\frac{-X-q Y}{1-q / t}\right] h_{\ell}\left[\frac{t^{n} X / q^{n}+t^{n+1} Y / q^{n}}{1-q / t}\right] \\
& =\left(\frac{1}{q t}\right)^{n(n-1) / 2} h_{n}\left[[n]_{q, t} X+[n+1]_{q, t} Y\right] .
\end{aligned}
$$


In general the action of $\nabla^{B}$ on Schur functions, contrary to that of $\nabla$, is not positive (up to an overall sign) when expanded in the Schur basis. But what is remarkable here is that the Schur expansion of the action of $\nabla^{B}$ on $s_{\emptyset ;(n)}$ can be given in a closed form for $q$ and $t$ generic (while a similar result for $\nabla e_{n}$ can only be established when $q=1 / t)$.

Corollary 18 The action of $\nabla^{B}$ on $s_{\emptyset ;(n)}$ expanded in the Schur basis $s_{\lambda, \mu}=$ $s_{\lambda}(x) s_{\mu}(y)$ is given by

$$
\nabla^{B} s_{\emptyset ;(n)}=\frac{1}{(q t)^{\left(\begin{array}{c}
n \\
2
\end{array}\right)}} \sum_{\lambda, \mu} s_{\lambda}\left[[n]_{q, t}\right] s_{\mu}\left[[n+1]_{q, t}\right] s_{\lambda, \mu} .
$$

In particular, the Schur-expansion coefficients belong to $\mathbb{N}\left[q^{ \pm 1}, t^{ \pm 1}\right]$.

Proof Using (4.9) and the Cauchy identity (see [21] and e.g., [16, eq. (1.63)]) $h_{n}[X Y]=\sum_{\mu \vdash n} s_{\mu}[X] s_{\mu}[Y]$, we find

$$
\begin{aligned}
h_{n}\left[[n]_{q, t} X+[n+1]_{q, t} Y\right] & =\sum_{\ell=0}^{n} h_{n-\ell}\left[[n]_{q, t} X\right] h_{\ell}\left[[n+1]_{q, t} Y\right] \\
& =\sum_{\ell=0}^{n} \sum_{\lambda \vdash n-\ell} s_{\lambda}\left[[n]_{q, t}\right] s_{\lambda}[X] \sum_{\mu \vdash \ell} s_{\mu}\left[[n+1]_{q, t}\right] s_{\mu}[Y] \\
& =\sum_{\lambda, \mu} s_{\lambda}\left[[n]_{q, t}\right] s_{\mu}\left[[n+1]_{q, t}\right] s_{\lambda, \mu} .
\end{aligned}
$$

The corollary then follows from Proposition 17. The positivity is immediate since $[n]_{q, t} \in \mathbb{N}[q, t]$ and the Schur functions are monomial positive.

The $B_{n}$ analog of the Schur functions is orthonormal

$$
\left\langle s_{\lambda, \mu}, s_{\omega, \nu}\right\rangle_{B}=\delta_{\lambda \omega} \delta_{\mu \nu}
$$

with respect to the $B_{n}$ analog of the Hall scalar product defined as ${ }^{8}$

$$
\left\langle p_{\lambda}[X+Y] p_{\mu}[X-Y], p_{\omega}[X+Y] p_{\nu}[X-Y]\right\rangle_{B}=\delta_{\lambda \omega} \delta_{\mu \nu} z_{\lambda} z_{\mu} 2^{\ell(\lambda)+\ell(\mu)} .
$$

We now extract from the previous Corollary a closed-form expression for the $B_{n}$ analog of the $q, t$-Catalan $\left\langle\nabla^{B} e_{n}, e_{n}\right\rangle$ (compare for instance with (4.3) in the case $q=1 / t$ ). As expected, it reduces to $\left(\begin{array}{c}2 n \\ n\end{array}\right)$ when $q=t=1$.

\footnotetext{
8 The scalar product is given in [21, Appendix B]. To be more specific, it is the specialization of $\left(5.3^{\prime}\right)$ therein to the case $B_{n} \cong C_{2} \sim S_{n}$ (discussed in more details in the example on page 178). The power of 2 is due to the fact that the centralizer of each element in $C_{2}$ is of order 2 .
} 


\section{Corollary 19}

$$
\left\langle\nabla^{B} s_{\emptyset ;(n)}, s_{\emptyset ;(n)}\right\rangle_{B}=\frac{1}{(q t)^{\left(\begin{array}{c}
n \\
2
\end{array}\right)}}\left[\begin{array}{c}
2 n \\
n
\end{array}\right]_{q, t},
$$

where

$$
\left[\begin{array}{c}
2 n \\
n
\end{array}\right]_{q, t}=\frac{[2 n]_{q, t} !}{[n]_{q, t} ![n]_{q, t} !} \quad \text { and } \quad[n]_{q, t} !=[n]_{q, t} \cdots[2]_{q, t}[1]_{q, t} .
$$

\section{In particular}

$$
\left\langle\nabla_{q=1 / t}^{B} s_{\emptyset ;(n)}, s_{\emptyset ;(n)}\right\rangle_{B}=\frac{1}{t^{n^{2}}}\left[\begin{array}{c}
2 n \\
n
\end{array}\right]_{t^{2}} .
$$

Proof Given the orthonormality (4.13), to evaluate $\left\langle\nabla^{B} s_{\emptyset ;(n)}, s_{\emptyset ;(n)}\right\rangle_{B}$, it suffices to compute the coefficient of $s_{\emptyset,(n)}$ in $\nabla^{B} s_{\emptyset,(n)}$. This coefficient corresponds to specifying $\lambda=\emptyset$ and $\mu=(n)$ in the r.h.s of (4.12):

$$
\left\langle\nabla^{B} s_{\emptyset ;(n)}, s_{\emptyset ;(n)}\right\rangle_{B}=\frac{1}{(q t)^{\left(\begin{array}{c}
n \\
2
\end{array}\right)}} h_{n}\left[[n+1]_{q, t}\right]=\frac{1}{(q t)^{\left(\begin{array}{c}
n \\
2
\end{array}\right)}}\left[\begin{array}{c}
2 n \\
n
\end{array}\right]_{q, t} .
$$

The previous equality follows from $[n+1]_{q, t}=q^{n}[n+1]_{t / q}$, the relation

$$
\left[\begin{array}{c}
2 n \\
n
\end{array}\right]_{q, t}=q^{n^{2}}\left[\begin{array}{c}
2 n \\
n
\end{array}\right]_{t / q}
$$

and the identity [21]

$$
h_{n}\left[[n+1]_{t}\right]=\left[\begin{array}{c}
2 n \\
n
\end{array}\right]_{t}
$$

Corollary 20 We have

$$
\left\langle\nabla^{B} s_{\emptyset ;(n)}, s_{\left(1^{n}\right) ; \emptyset}\right\rangle_{B}=1
$$

Proof As in the proof of the previous corollary, it suffices to compute the coefficient of $s_{\left(1^{n}\right), \emptyset}$ in $\nabla^{B} s_{\emptyset,(n)}$, which corresponds to specifying $\lambda=\left(1^{n}\right)$ and $\mu=\emptyset$ in the r.h.s of (4.12):

$$
\begin{aligned}
\left\langle\nabla^{B} s_{\emptyset ;(n)}, s_{\left(1^{n}\right), \emptyset}\right\rangle_{B} & =\frac{1}{(q t)^{\left(\begin{array}{c}
n \\
2
\end{array}\right)}} s_{1^{n}}\left[[n]_{q, t}\right] \\
& =\frac{1}{(q t)^{\left(\begin{array}{c}
n \\
2
\end{array}\right)}}\left(q^{n-1}\right)\left(q^{n-2} t\right) \cdots\left(q t^{n-2}\right)\left(t^{n-1}\right)=1 .
\end{aligned}
$$


The final result of this section is an explicit expression for the analog of $\left\langle\nabla e_{n}, p_{1}^{n}\right\rangle$ given by $\left\langle\nabla^{B} s_{\emptyset,(n)}, p_{\emptyset,\left(1^{n}\right)}\right\rangle_{B}$ (where we recall that $\left.p_{\emptyset,\left(1^{n}\right)}=p_{1^{n}}[X+Y]\right)$. Observe the similarity with (4.1) when $q=1 / t$. Quite unexpectedly, the present expression, a priori more complicated, factorizes for $q$ and $t$ generic (recall the discussion surrounding Eq. (4.2) pertaining to the non-factorization in the usual case).

Proposition 21 We have

$$
\left\langle\nabla^{B} s_{\emptyset,(n)}, p_{\emptyset,\left(1^{n}\right)}\right\rangle_{B}=\left(\frac{[n+1]_{q, t}+[n]_{q, t}}{(q t)^{(n-1) / 2}}\right)^{n} .
$$

In particular, there follows the two specializations:

$$
\left\langle\nabla_{q=1 / t}^{B} s_{\emptyset,(n)}, p_{\emptyset,\left(1^{n}\right)}\right\rangle_{B}=\left(\frac{[2 n+1]_{t}}{t^{n}}\right)^{n}
$$

and

$$
\left\langle\nabla_{q=t=1}^{B} s_{\emptyset,(n)}, p_{\emptyset,\left(1^{n}\right)}\right\rangle_{B}=(2 n+1)^{n} .
$$

Proof We first need to obtain the coefficient $p_{1^{n}}[X+Y]$ in the expansion of $\nabla^{B} s_{\emptyset,(n)}$ in the $p_{\lambda}[X-Y] p_{\mu}[X+Y]$ basis. We have

$$
\begin{aligned}
{[n]_{q, t} X+[n+1]_{q, t} Y=} & \frac{1}{2}\left([n]_{q, t}+[n+1]_{q, t}\right)(X+Y) \\
& +\frac{1}{2}\left([n]_{q, t}-[n+1]_{q, t}\right)(X-Y)
\end{aligned}
$$

and thus this amounts, from Proposition 17, to computing the coefficient of $p_{1^{n}}[X+Y]$ in

$$
\begin{aligned}
& \frac{1}{(q t))^{\left(\begin{array}{c}
n \\
2
\end{array}\right)}} h_{n}\left[\frac{1}{2}\left([n]_{q, t}+[n+1]_{q, t}\right)(X+Y)+\frac{1}{2}\left([n]_{q, t}-[n+1]_{q, t}\right)(X-Y)\right] \\
& =\frac{1}{(q t)} \sum_{\lambda, \mu} \frac{1}{z_{\lambda} z_{\mu}} p_{\lambda}\left[\frac{1}{2}\left([n]_{q, t}+[n+1]_{q, t}\right)\right] p_{\mu}\left[\frac{1}{2}\left([n]_{q, t}-[n+1]_{q, t}\right)\right] \\
& \quad p_{\lambda}[X+Y] p_{\mu}[X-Y],
\end{aligned}
$$

where we used (4.9), the expansion $h_{n}=\sum_{\lambda \vdash n} p_{\lambda} / z_{\lambda}$ ([21, eq. I.2.14']), and the basic property $p_{\lambda}[X Y]=p_{\lambda}[X] p_{\lambda}[Y]$. Letting $\lambda=\left(1^{n}\right)$ and $\mu=\emptyset$, the coefficient of $p_{1^{n}}[X+Y]$ is easily found to be

$$
\begin{aligned}
& \frac{1}{(q t)^{\left(\begin{array}{c}
n \\
2
\end{array}\right)} z_{1^{n}}} p_{1^{n}}\left[\frac{1}{2}\left([n]_{q, t}+[n+1]_{q, t}\right)\right]=\frac{1}{(q t)^{\left(\begin{array}{c}
n \\
2
\end{array}\right)} n !}\left(p_{1}\left[\frac{1}{2}\left([n]_{q, t}+[n+1]_{q, t}\right)\right]\right)^{n} \\
& =\frac{1}{2^{n} n !}\left(\frac{[n]_{q, t}+[n+1]_{q, t}}{(q t)^{(n-1) / 2}}\right)^{n} .
\end{aligned}
$$


The proposition then follows from

$$
\left\langle p_{1^{n}}[X+Y], p_{1^{n}}[X+Y]\right\rangle_{B}=2^{n} n !
$$

Remark 22 As mentioned in the introduction, $\nabla_{q=t^{-1}}^{B} s_{\emptyset ;(n)}$ seems to coincide with Frob $_{t^{-1}, t}\left(R^{B_{n}}\right)$, where $R^{B_{n}}$ is a certain doubly graded quotient of the coinvariant ring $C^{B_{n}}$. From Remark 13, one would be tempted to believe that there exists for every pair of partitions $(\lambda, \mu)$, a bigraded submodule of $R^{B_{n}}$ (isomorphic to the regular module of the hyperoctahedral group $B_{n}$ ) whose Frobenius series when $q=t^{-1}$ corresponds to the Schur expansion of $\tilde{H}_{\lambda, \mu}\left(x, y ; t^{-1}, t\right)$. This cannot be the case, however, since $\nabla^{B} s_{\emptyset ;(n)}-\tilde{H}_{\lambda, \mu}(x, y ; q, t)$ is not Schur-positive in general (even when $\left.q=t^{-1}\right)$.

Remark 23 The Macdonald polynomials $\tilde{H}_{\lambda}^{(q, t)}[X]$ can be defined (up to normalization constants) as the unique basis such that

(i) $\tilde{H}_{\lambda}^{(q, t)}[X(1-t)] \in \mathbb{Q}(q, t)\left\{s_{\mu}: \mu \leq \lambda\right\}$,

(ii) $\tilde{H}_{\lambda}^{(q, t)}[X(1-q)] \in \mathbb{Q}(q, t)\left\{s_{\mu}: \mu \geq \lambda\right\}$.

The double Macdonald polynomials can also be defined by two similar triangularities. Let $\phi_{t}$ be the plethystic substitution

$$
X \mapsto X \quad \text { and } \quad(X+Y) \mapsto(X+Y)(1-t)
$$

and recall that $\omega_{B}$ is the involution such that $\omega_{B} s_{\lambda, \mu}(x, y)=s_{\mu^{\prime}, \lambda^{\prime}}(x, y)$. The double Macdonald polynomials $\tilde{H}_{\lambda, \mu}(x, y ; q, t)$ can be characterized (up to normalization constants) as the unique basis of the space of bisymmetric functions such that

(i) $\phi_{t} \tilde{H}_{\lambda, \mu}(x, y ; q, t) \in \mathbb{Q}(q, t)\left\{s_{\omega, \eta}:(\omega, \eta) \leq(\lambda, \mu)\right\}$,

(ii) $\omega_{B} \circ \phi_{q} \circ \omega_{B} \tilde{H}_{\lambda, \mu}(x, y ; q, t) \in \mathbb{Q}(q, t)\left\{s_{\omega, \eta}:(\omega, \eta) \geq(\lambda, \mu)\right\}$.

The first triangularity is seen as follows: $\phi_{t} \tilde{H}_{\lambda, \mu}(x, y ; q, t)$ is equal up to a constant to $P_{\lambda, \mu}\left(x, y ; q, t^{-1}\right)$, which is lower triangular in the $m_{\lambda, \mu}$ basis and, hence, in the $s_{\lambda, \mu}$ basis (the $s_{\lambda, \mu}$ basis is lower triangular in the $m_{\lambda, \mu}$ basis since it corresponds to the specialization $q=t=0$ of $\left.P_{\lambda, \mu}(x, y ; q, t)\right)$. Using the symmetry (3.36), we have immediately that $\tilde{H}_{\lambda, \mu}(x, y ; q, t)$ is upper triangular in the $s_{\lambda, \mu}$ basis. The two triangularities in the double case are not as symmetric as in the usual case due to the noncommutativity of $\omega_{B}$ and the plethystic substitution $\phi_{q}$ (whereas in the usual case, the involution $\omega$ commutes with any plethystic substitution).

Haiman also introduced in [19] wreath Macdonald polynomials. In the special case of the complex reflection group $G(2,1, n)$, the wreath Macdonald polynomial $\mathcal{H}_{\mu}(q, t)$ depends on a choice of staircase partition $\delta^{m}$ (a 2-core) and is indexed by a partition $\mu$ of size $m(m-1) / 2+n$, where $n$ is a fixed integer. They satisfy the triangularities 


$$
\mathcal{H}_{\mu}(q, t) \otimes \sum_{i}(-q)^{i} \operatorname{char}\left(\wedge^{i} \mathfrak{h}\right) \in \mathbb{Q}(q, t)\left\{\chi^{\mathrm{Quot}_{2}(\lambda)}: \lambda \geq \mu, \operatorname{Core}_{2}(\lambda)=\delta^{m}\right\}
$$

and

$$
\mathcal{H}_{\mu}(q, t) \otimes \sum_{i}(-t)^{i} \operatorname{char}\left(\wedge^{i} \mathfrak{h}\right) \in \mathbb{Q}(q, t)\left\{\chi^{\mathrm{Quot}_{2}(\lambda)}: \lambda \leq \mu, \operatorname{Core}_{2}(\lambda)=\delta^{m}\right\}
$$

where $\mathfrak{h}=\mathbb{C}^{n}$ is the defining representation of $G(2,1, n)$, Quot $2(\lambda)$ is the 2-quotient of $\lambda$ (a pair of partitions of total degree $n$ ), and $\operatorname{Core}_{2}(\lambda)$ is the 2-core of $\lambda$. This is somewhat reminiscent of our construction, even more so that if $m$ is large enough Haiman claims that the corresponding wreath Macdonald polynomials can be given in terms of usual Macdonald polynomials. However, the objects cannot coincide given that the symmetry in the triangularities (4.17) and (4.18) is not present in the double case.

\section{Conclusion: the superspace bridge}

The present construction relies on the formalism previously developed for Macdonald superpolynomials $[6,7]$. To these superpolynomials, we have associated bisymmetric polynomials by considering the coefficient of a given monomial in the $\theta_{i}$ 's, say $\theta_{1} \ldots \theta_{m}$ for a superpolynomial of fermionic degree $m$, and dividing by the Vandermonde determinant in the commuting variables $x_{1}, \ldots, x_{m}$. We have seen that for sufficiently high fermionic degree, a stable sector is reached. In this stable sector, our key result is the product form (3.10). As a consequence of this remarkable factorization, we have been able to prove rather directly a number of properties for these so-called double Macdonald polynomials. In particular, we have obtained

- The expression for the norm.

- The expression for the integral form.

- The evaluation.

- The positivity and integrability of the double Kostka coefficients.

- The two Macdonald-type symmetry properties of the double Kostka coefficients.

In $[6,7]$, we have presented conjectures related to the above five items but pertaining to generic Macdonald superpolynomials. The present results imply that we now have proofs of these results for all cases where $m \geq n$ (in some sense for roughly half the cases). The precise connection between the present results and our more general conjectures is worked out in Appendix C.

We had a number of mathematical and physical motivations for undertaking the study of the Macdonald superpolynomials. One of which, of a combinatorial nature, was to see whether by adding more structure to the usual Macdonald polynomials, one could get unexpected new handles on open problems such as a combinatorial description of the $q, t$-Kostka coefficients, generalizing the Lascoux-Schützenberger 
description of the Kostka-Foulkes coefficients. For the stable sector considered here, this particular hope was not fulfilled: our new double Kostka coefficients are roughly sums of products of the usual ones.

However, we have already presented a conjectural result-pertaining to the nonstable sector, which thereby necessarily relies on the superspace formalism - that could shed some light on the combinatorics of the usual $q$, $t$-Kostka coefficients [7]. It says that the simplest superpolynomials, namely those in the $m=1$ sector, do provide a refinement of the Kostka coefficients. Precisely, this conjectural result gives a relation between the generalized coefficients $K_{\Omega \Lambda}(q, t)$ of fermionic degree $m=1$ and total degree $n$ and the usual $q, t$-Kostka coefficients of degree $n+1$.

Conjecture 24 Let $\Lambda$ be a superpartition of fermionic degree $m=1$, and let $H_{\Lambda}$ be the modified Macdonald superpolynomial (see Appendix C for more details). Let also $\psi$ be the linear application that maps $s_{\Omega}$ to $s_{\Omega} \circledast$. Then

$$
\psi\left(H_{\Lambda}\right)=H_{\Lambda \circledast} .
$$

This conjecture implies that the usual $q, t$-Kostka coefficient $K_{\mu \lambda}(q, t)$ can be calculated from its lower degree super-relatives as

$$
K_{\mu \lambda}(q, t)=\sum_{\Omega \mid \Omega^{\circledast}=\mu} K_{\Omega \Lambda}(q, t)
$$

where $\Lambda$ is any superpartition that can be obtained from $\lambda$ by replacing a square by a circle, and the sum is over all $\Omega$ 's that can be obtained from $\mu$ by replacing a square by a circle. Moreover, the expression for the sum on the right-hand side is independent of the choice of $\Lambda$. We thus relate a Kostka coefficient of a given degree to a sum of lower degree Kostka coefficients in the $m=1$ fermionic sector, a process that mimics a sort of transmutation of the fermionic variable into a bosonic one.

For example, consider $H_{(2 ; 1)}(x, \theta ; q, t)$. Its Schur expansion reads (using the diagrammatic representation of superpartitions introduced in Appendix B)

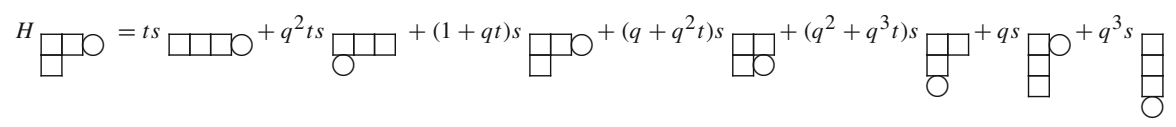

Now apply $\psi$ :

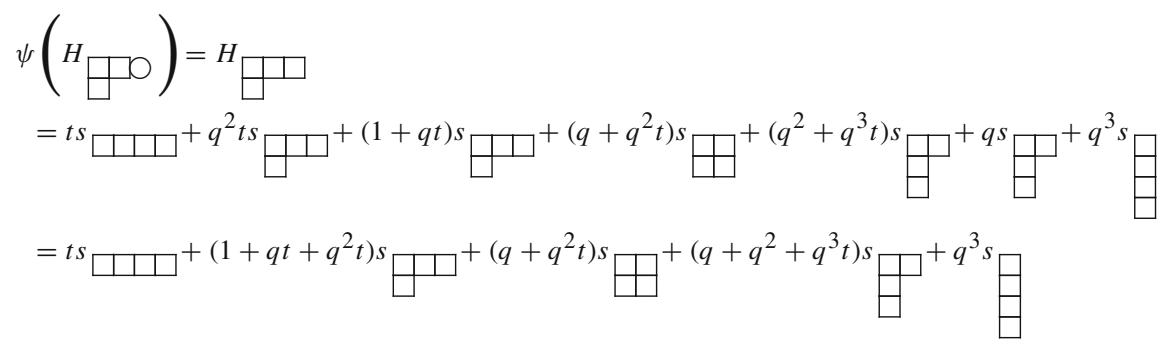


which corresponds to the usual Schur expansion of the modified Macdonald polynomials $H_{(3,1)}(x ; q, t)$.

This conjecture can be generalized as follows. Let $\Lambda$ and $\Lambda^{\square}$ be two superpartitions such that $\Lambda^{\square}$ is obtained from $\Lambda$ by replacing a circle by a box. Note that $\Lambda^{\square}$ is not unique for $m>1$. Define $\#\left(\Lambda, \Lambda^{\square}\right)$ to be the number of circles that lie above the row of $\Lambda$, where the circle has been replaced by a box.

Conjecture 25 Let $\widehat{H}_{\Lambda}$ be the normalized modified Macdonald superpolynomial defined as

$$
\widehat{H}_{\Lambda}=v_{\Lambda} H_{\Lambda}, \quad \text { with } \quad v_{\Lambda}=\prod_{s \in \Lambda / \mathcal{B} \Lambda}\left(1-q^{a_{\Lambda} \circledast(s)} t^{l_{\Lambda^{*}}(s)+1}\right)
$$

and let $\psi$ be the linear application that maps

$$
\psi: s_{\Lambda} \mapsto \psi\left(s_{\Lambda}\right)=\sum_{\Omega \mid \Omega=\Lambda^{\square}}(-1)^{\#(\Lambda, \Omega)} s_{\Omega}
$$

Then, we have

$$
\psi\left(\widehat{H}_{\Lambda}\right)=\sum_{\Omega \mid \Omega=\Lambda^{\square}}(-1)^{\#(\Lambda, \Omega)} \widehat{H}_{\Omega}
$$

Note that if $\Lambda$ has fermionic degree $m=1$, then this reduces to the previous conjecture since then all the factors $v_{\bullet}$ reduce to 1 . We stress that $\Lambda / \mathcal{B} \Lambda$ refers to the set of boxes in $\Lambda$ that are not in $\mathcal{B} \Lambda$, a set denoted by $\mathcal{F} \Lambda$ in Appendix $\mathrm{C}$ (see below Eq. (8.14)). This set is empty when $m=1$.

Remark 26 It is straightforward to check that the application $\psi$ is such that $\psi \circ \psi=0$. In the language of [11], $\psi$ corresponds to $\sum_{i} x_{i} \partial_{\theta_{i}}$, the adjoint of the exterior derivative $\sum_{i} \theta_{i} \partial_{x_{i}}$.

Let us make explicit the implication of this result at the level of the Kostkas. We have

$$
\psi\left(\widehat{H}_{\Lambda}\right)=v_{\Lambda} \sum_{\Omega} K_{\Omega \Lambda}(q, t) \psi\left(s_{\Omega}\right)=v_{\Lambda} \sum_{\Omega} K_{\Omega \Lambda}(q, t) \sum_{\Delta \mid \Delta=\Omega^{\square}}(-1)^{\#(\Omega, \Delta)} s_{\Delta}
$$

and

$$
\psi\left(\widehat{H}_{\Lambda}\right)=\sum_{\Gamma \mid \Gamma=\Lambda^{\varpi}}(-1)^{\#(\Lambda, \Gamma)} \widehat{H}_{\Gamma}=\sum_{\Gamma \mid \Gamma=\Lambda^{\square}}(-1)^{\#(\Lambda, \Gamma)} v_{\Gamma} \sum_{\Delta} K_{\Delta, \Gamma} s_{\Delta} .
$$

By comparing the coefficients of $s_{\Delta}$, we get

$$
v_{\Lambda} \sum_{\Omega \mid \Omega^{\square}=\Delta}(-1)^{\#(\Omega, \Delta)} K_{\Omega \Lambda}(q, t)=\sum_{\Gamma \mid \Gamma=\Lambda^{\square}}(-1)^{\#(\Lambda, \Gamma)} v_{\Gamma} K_{\Delta, \Gamma} .
$$


This is thus a relation between a linear combination of Kostkas for fermionic degrees differing by 1 . The case $m=1$ is of course special in that the sum on the r.h.s. reduces to a single term. As already said, when $m>1, \Lambda^{\square}$ can take $m$ different values, and therefore the r.h.s. sum contains $m$ terms. Let us then reverse the point of view and see whether there are situations for which the Kostkas at fermionic degree $m$ could be computed from those at degree $m-1$. This would be the case if the sum on the l.h.s. could be reduced to a single term. This is actually the case when the diagram of $\Delta$ has a single removable box. This removable box is thus necessarily the one created when a circle is changed into a box.

Consider an example. Take $\Delta=(3 ; 2)$, which has one removable box (indicated by $\times)$ so that $\Omega=(3,1$; ):

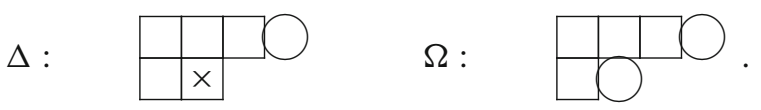

The 1.h.s. of (5.2) becomes $v_{\Lambda}(-1) K_{(3,1 ;), \Lambda}(q, t)$. Let us set $\Lambda=(2,0 ; 2): \Gamma$ in the sum of the r.h.s. of (5.2) can take the two values $(1 ; 3,2)$ and $(2 ; 2,1)$ :

$\Lambda:$

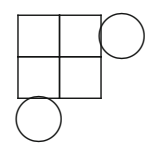

$\stackrel{\psi}{\rightarrow} \quad \Lambda^{\square}:$

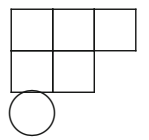

and

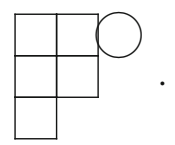

Hence, the r.h.s. of (5.2) reduces to $K_{(3 ; 2)(0 ; 3,2)}(q, t)-K_{(3 ; 2)(2 ; 2,1)}(q, t)$. We thus end up with the relation

$$
-\left(1-q^{2} t^{2}\right) K_{(3,1 ;),(2,0 ; 2)}(q, t)=K_{(3 ; 2)(0 ; 3,2)}(q, t)-K_{(3 ; 2)(2 ; 2,1)}(q, t) .
$$

With

$$
\begin{aligned}
& K_{(3 ; 2)(0 ; 3,2)}(q, t)=t+t^{2}+q t^{2}+q t^{3}+q^{2} t^{4} \text { and } \\
& K_{(3 ; 2)(2 ; 2,1)}(q, t)=t^{2}\left(1+q t+q^{2} t+q^{2} t^{2}+q^{3} t^{2}\right)
\end{aligned}
$$

we get

$$
\begin{aligned}
-\left(1-q^{2} t^{2}\right) K_{(3,1 ;),(2,0 ; 2)}(q, t) & =-\left(1-q^{2} t^{2}\right)\left(t+q t^{2}\right) \\
& \Rightarrow \quad K_{(3,1 ;),(2,0 ; 2)}(q, t)=t+q t^{2}
\end{aligned}
$$

Another instance where this relation appears to be useful is when $\Delta$ has no removable box. In this case, there are no $\Omega$ such that $\Omega^{\square}=\Delta$, so that the sum on the 1.h.s. of (5.2) vanishes. The r.h.s. becomes an identity on alternating sums of Kostkas weighted by the factor $v_{\Gamma}$. Here is an example: take $\Delta=(3,2 ;)$, which clearly has no removable box, and $\Lambda=(3,1,0 ;)$ 


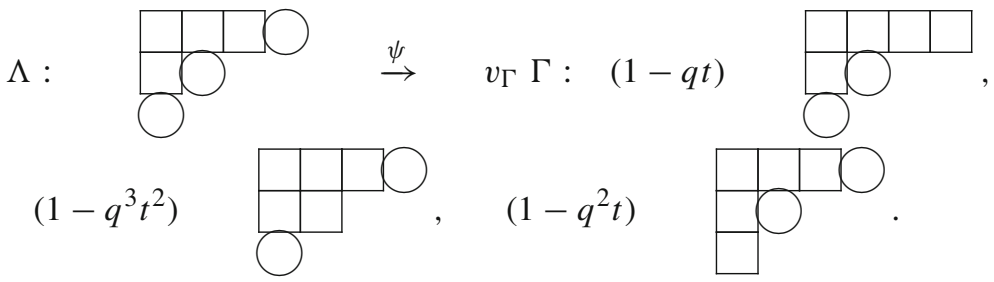

This leads to the relation

$$
\begin{aligned}
& (1-q t) K_{(3,2 ;),(1,0 ; 4)}(q, t)-\left(1-q^{3} t^{2}\right) K_{(3,2 ;),(3,0 ; 2)}(q, t) \\
& \quad+\left(1-q^{2} t\right) K_{(3,2 ;),(3,1 ; 1)}(q, t)=0 .
\end{aligned}
$$

With

$$
\begin{aligned}
& K_{(3,2 ;),(1,0 ; 4)}(q, t)=q^{2} t^{2}\left(1+q^{2} t\right) \quad \text { and } \\
& K_{(3,2 ;),(3,0 ; 2)}(q, t)=K_{(3,2 ;),(3,1 ; 1)}(q, t)=t\left(1+q^{2} t\right),
\end{aligned}
$$

this is easily checked to be satisfied.

Acknowledgments The authors are extremely grateful to Patrick Desrosiers and Stephen Griffeth for helpful discussions, and to Mark Haiman for sharing his data on wreath Macdonald polynomials. This work was supported by NSERC, FQRNT, FONDECYT (Fondo Nacional de Desarrollo Científico y Tecnológico de Chile) grant \#1130696, and by CONICYT (Comisión Nacional de Investigación Científica y Tecnológica de Chile) via the "proyecto anillo ACT56".

\section{Appendix A: Monomials and power sums in superspace in bisymmetric form}

The following proposition corresponds to formulas (128) and (176) of [10]. Since it concerns basic results underlying our construction, we nevertheless include its proof for completeness.

Proposition 27 The bisymmetric monomial deduced from the supermonomial of fermionic degree $m$ is given by

$$
m_{\lambda, \mu}\left(x_{1}, \ldots, x_{N}\right)=s_{\lambda}\left(x_{1}, \ldots, x_{m}\right) m_{\mu}\left(x_{m+1}, \ldots x_{N}\right),
$$

where $s_{\lambda}$ and $m_{\lambda}$ are, respectively, the Schur polynomials and the usual monomial functions.

Similarly, the bisymmetric power-sum reads

$$
p_{\lambda, \mu}\left(x_{1}, \ldots, x_{N}\right)=s_{\lambda}\left(x_{1}, \ldots, x_{m}\right) p_{\mu}\left(x_{1}, \ldots, x_{N}\right) .
$$

Note that in the first case, the two functions on the r.h.s depend upon distinct set of variables, which is not the case in the expression of the power sums. 
Proof Let us first establish (6.1), starting with the expression for the supermonomial

$$
m_{\Lambda}=\frac{1}{f_{\Lambda^{s}}(1)} \sum_{\sigma \in S_{N}} \mathcal{K}_{\sigma} \theta_{1} \ldots \theta_{m} x^{\Lambda}, \quad \text { where } \quad f_{\Lambda^{s}}(1)=\prod_{i \geq 0} n_{\Lambda^{s}}(i) !
$$

with $n_{\Lambda^{s}}(i)$ being the number of occurrences of $i$ in $\Lambda^{s}$, and $m$ being the number of fermions. The permutation $\mathcal{K}_{i j}$ interchanges the pairs $\left(x_{i}, \theta_{i}\right)$ and $\left(x_{j}, \theta_{j}\right)$. Focusing on the term of $m_{\Lambda}$ that contains $\theta_{1} \ldots \theta_{m}$, we have

$$
\left[\theta_{1} \ldots \theta_{m}\right] m_{\Lambda}=\frac{1}{f_{\Lambda^{s}}(1)} \mathcal{A}_{1, \ldots, m} \mathcal{S}_{m+1, \ldots, N} x^{\Lambda},
$$

where $\mathcal{A}$ is the anti-symmetrizing operator (which, here, acts on the variables $x_{1} \cdots x_{m}$ ) and $\mathcal{S}$ is the symmetrizing operator (acting on the variables $x_{m+1} \cdots, x_{N}$ ). The coefficient $f_{\Lambda^{s}}(1)$ ensures that repeated terms count for 1 in the expression. Since $\mathcal{A}$ and $\mathcal{S}$ are independent operators (acting on different sets of variables), we can write

$$
\begin{aligned}
{\left[\theta_{1} \ldots \theta_{m}\right] m_{\Lambda}=} & \frac{1}{f_{\Lambda^{s}}(1)}\left(\sum_{w \in S_{m}} \varepsilon(w) K_{w}\left(x_{1}^{\Lambda_{1}^{a}} \ldots x_{m}^{\Lambda_{m}^{a}}\right)\right) \\
& \left(\sum_{\sigma \in S_{N-m}} K_{\sigma}\left(x_{m+1}^{\Lambda_{1}^{s}} \ldots x_{N}^{\Lambda_{N-m}^{s}}\right)\right)
\end{aligned}
$$

where $\varepsilon(w)$ is the sign of permutation $w$ and $K_{i j}$ interchanges $x_{i}$ and $x_{j}$. By dividing this expression by the Vandermonde determinant in the variables $x_{1} \cdots x_{m}$, denoted $\Delta_{m}$, and using the decomposition $\Lambda=\left(\Lambda^{a} ; \Lambda^{s}\right)=\left(\lambda+\delta^{m} ; \mu\right)$, we obtain

$$
\begin{aligned}
{\left[\theta_{1} \ldots \theta_{m}\right] \frac{1}{\Delta_{m}} m_{\Lambda}=} & \left(\frac{\sum_{w \in S_{m}} \varepsilon(w) K_{w}\left(\bar{x}^{\delta^{m}+\lambda}\right)}{\prod_{1 \leq i<j \leq m}\left(x_{i}-x_{j}\right)}\right) \\
& \left(\frac{1}{f_{\mu}(1)} \sum_{\sigma \in S_{N-m}} K_{\sigma}\left(x_{m+1}^{\mu_{1}} \ldots x_{N}^{\mu_{N-m}}\right)\right)=: m_{\lambda, \mu},
\end{aligned}
$$

where $\bar{x}$ denote the variables $x_{1}, \ldots, x_{m}$. The term in the first parenthesis is nothing but the definition of a Schur function in the variable $x_{1} \ldots x_{m}$, that is $s_{\lambda}\left(x_{1}, \ldots, x_{m}\right)$. The second term is simply the monomial function in the other variables over the partition $\mu$, that is, $m_{\mu}\left(x_{m+1}, \ldots, x_{N}\right)$. We have thus recovered (6.1).

For the derivation of (6.2), we proceed in a similar way. The complete power-sum superfunction labeled by $\Lambda$ reads

$$
p_{\Lambda}=\tilde{p}_{\Lambda_{1}^{a}} \ldots \tilde{p}_{\Lambda_{m}^{a}} p_{\Lambda_{1}^{s}} \ldots p_{\Lambda_{N-m}^{s}}
$$

where $\tilde{p}_{k}=\sum_{i=1}^{N} \theta_{i} x_{i}^{k}$ and $p_{k}=\sum_{i=1}^{N} x_{i}^{k}$. Taking the coefficient in $\theta_{1} \cdots \theta_{m}$, we have 


$$
\left[\theta_{1} \ldots \theta_{m}\right] p_{\Lambda}=\left(\mathcal{A}_{1, \ldots, m} \bar{x}^{\Lambda^{a}}\right) p_{\Lambda^{s}}\left(x_{1}, \ldots, x_{N}\right)
$$

Dividing by the Vandermonde in the $m$ first variables and we finally obtain

$$
\left[\theta_{1} \cdots \theta_{m}\right] \frac{1}{\Delta_{m}} p_{\Lambda}=\left(\frac{\sum_{w \in S_{m}} \varepsilon(w) K_{w}\left(\bar{x}^{\delta^{m}+\lambda}\right)}{\prod_{1 \leq i<j \leq m}\left(x_{i}-x_{j}\right)}\right) p_{\mu}\left(x_{1}, \ldots, x_{N}\right)=: p_{\lambda, \mu}
$$

where the term in parenthesis is the Schur functions $s_{\lambda}\left(x_{1}, \ldots, x_{m}\right)$. This gives (6.2).

\section{Appendix B: Induced properties on pairs of partitions: conjugation and domi- nance order}

As mentioned in the introduction, a superpartition is a pair of partitions of the form $\Lambda=\left(\Lambda^{a} ; \Lambda^{s}\right)$, where the partition $\Lambda^{a}$ has $m$ distinct parts (the $m$-th one can be equal to 0$)$. We define

$$
\Lambda^{*}=\Lambda^{a} \cup \Lambda^{s} \text { and } \Lambda^{\circledast}=\left(\Lambda^{a}+1^{m}\right) \cup \Lambda^{s}
$$

It is manifest that the pair of partitions $\left(\Lambda^{\circledast}, \Lambda^{*}\right)$ fixes the superpartition $\Lambda$. A diagrammatic representation of $\Lambda$ is obtained from the Ferrers diagram of $\Lambda^{\circledast}$ by changing into circles the cells of $\Lambda^{\circledast} / \Lambda^{*}$. For instance, if $\Lambda=(3,1,0 ; 2,1)$, we have

$\Lambda^{\circledast}$ :

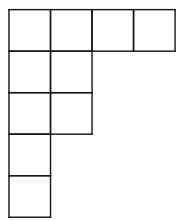

$\Lambda^{*}:$

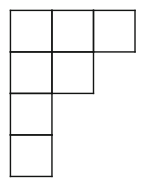

which gives $\Lambda$ :

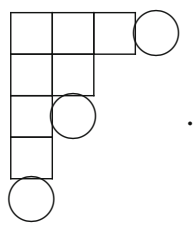

Recall that we can associate to a superpartition $\Lambda$ of fermionic degree $m$ a pair of partitions $\lambda$ and $\mu$ in the following way:

$$
\Lambda=\left(\Lambda^{a} ; \Lambda^{s}\right) \leftrightarrow(\lambda, \mu)=\left(\Lambda^{a}-\delta^{m}, \Lambda^{s}\right)
$$

When $m \geq n$, this establishes an obvious bijection between superpartitions $\left(\Lambda^{a} ; \Lambda^{s}\right)$ of fermionic degree $m$ such that $\left|\Lambda^{a}\right|+\left|\Lambda^{s}\right|=n+m(m-1) / 2$ and pairs of partitions $(\lambda, \mu)$ such that $|\lambda|+|\mu|=n$. Before describing how the conjugation and the dominance-ordering properties are induced from superpartitions to pairs of partitions, we establish some elementary results that will be used for this analysis. In that regard, it is convenient to first introduce a convention concerning the positions of the boxes corresponding to $\lambda$ within the diagrammatic representation of the superpartition $\Lambda$. We choose to place the boxes of $\lambda$ (the boxes marked with a $\circ$ in the example below) in columns that are not fermionic (that is, that do not end with a circle). For instance, 
consider $\lambda=(2,1), \mu=(3,1)$ and the corresponding $\Lambda$ for $m=3,4,5$ (all in the non-stable sector, illustrating the fact that the two partitions get disentangled before $m \geq n=7)$ :

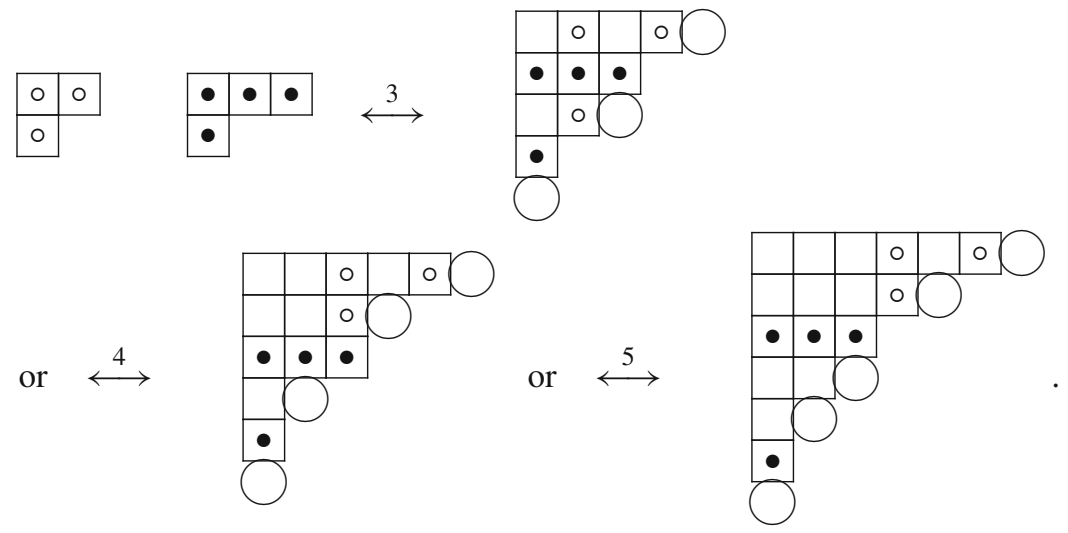

Lemma 28 Let $\lambda, \mu, \Lambda$, and $m$ be defined as in (7.2) and suppose that $m \geq n=$ $|\lambda|+|\mu|$. If $\ell(\lambda)=\ell$, then the $\ell$-th entry of $\Lambda^{a}$ is strictly larger than the first entry of $\Lambda^{s}$, that is,

$$
\Lambda_{\ell}^{a}>\Lambda_{1}^{s}=\mu_{1}
$$

In particular, in the diagram of the superpartition $\Lambda$, the cells marked with a $\circ$ appear strictly above those marked with a $\bullet$

Proof The condition $m \geq n$ implies

$$
m \geq \lambda_{1}+\cdots+\lambda_{\ell}+\mu_{1} \geq \ell+\mu_{1}>\ell-\lambda_{\ell}+\mu_{1} .
$$

Hence,

$$
\Lambda_{\ell}^{a}=\lambda_{\ell}+m-\ell>\mu_{1}=\Lambda_{1}^{s} .
$$

The bound $m \geq n$ is not the optimal one ensuring the separation of $\lambda$ and $\mu$ in $\Lambda$ but it is sufficient for our purpose.

Lemma 29 In the diagram of the superpartition $\Lambda$, when $m \geq n$, the cells marked with $a$ o appear strictly to the right of those marked with a

Proof By inspection, we see that the column in $\Lambda$ where the o corresponding to the first column of $\lambda$ are inserted is the $m-\lambda_{1}^{\prime}+1$ one. The lemma will then hold if $m-\lambda_{1}^{\prime}+1>\mu_{1}$. Given $\lambda_{1}^{\prime}=\ell(\lambda)=\ell$, this corresponds to $m \geq \ell+\mu_{1}$, which is a consequence of $m \geq n$ as seen in (7.4). 
We now come to the conjugation property.

Lemma 30 Suppose that as in (7.2), we have $\Lambda \leftrightarrow(\lambda, \mu)$ with $m \geq n$. Then

$$
\Lambda^{\prime} \leftrightarrow\left(\mu^{\prime}, \lambda^{\prime}\right)
$$

Proof This follows directly from the definition of the conjugation for superpartitions, obtained by the interchange of rows and columns.

An example will make this completely obvious: consider the pair $(2),(3,1)$, so that, with $m=6$,

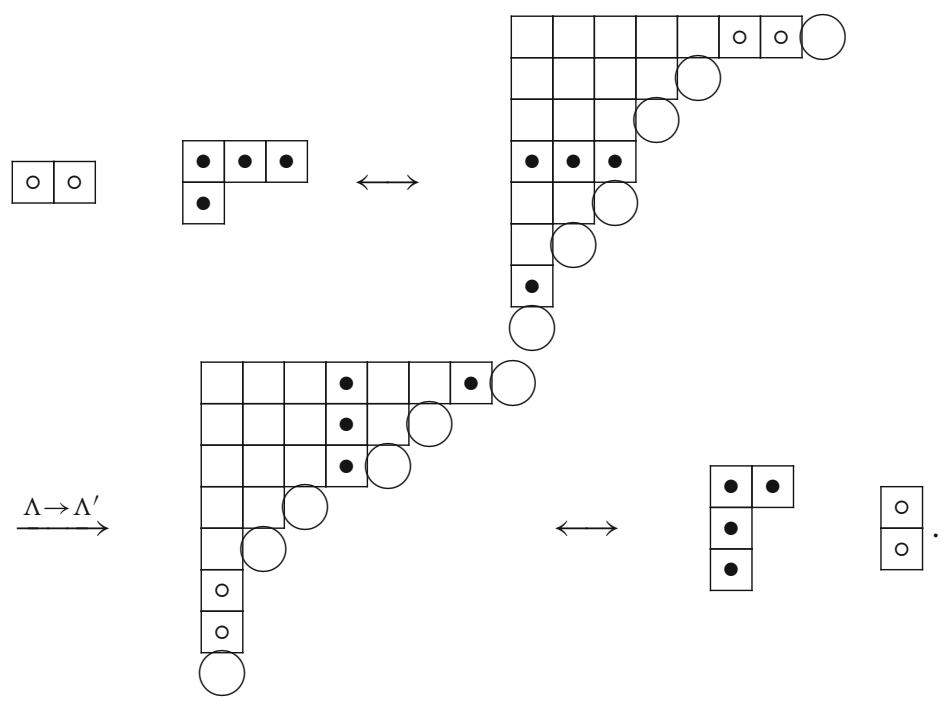

We observe that our convention of placing the boxes of the first partition into bosonic columns of $\Lambda$ is preserved by the conjugation operation.

Recall that the dominance ordering on bi-partitions was defined in (1.8). In the following lemma, we give three equivalent forms of the second condition in the dominance ordering on bi-partitions. For this purpose, it will prove convenient to relax the condition $|\lambda|=|\mu|$ in the dominance ordering and say that

$$
\lambda \geq \mu \quad \text { if } \quad \lambda_{1}+\cdots+\lambda_{j} \geq \mu_{1}+\cdots+\mu_{j} \quad \forall j,
$$

even in cases where $|\lambda| \neq|\mu|$. Observe, however, that the equivalence

$$
\lambda \geq \mu \Longleftrightarrow \lambda^{\prime} \leq \mu^{\prime}
$$

only holds if $|\lambda|=|\mu|$.

Lemma 31 Suppose that $|\lambda| \geq|\omega|$. Then, the following three statements are equivalent: 
(1) $|\lambda|+\mu \geq|\omega|+\eta$

(2) $\mu^{\prime} \cup\left(1^{|\lambda|-|\omega|}\right) \leq \eta^{\prime}$,

(3) $\mu^{\prime} \leq \eta^{\prime}$.

Proof We have that (1) and (2) are equivalent since

$$
\begin{aligned}
|\lambda|+\mu_{1}+\cdots+\mu_{j} \geq|\omega|+\eta_{1}+\cdots+\eta_{j} \quad \forall j & \Longleftrightarrow \mu+(|\lambda|-|\omega|) \geq \eta \\
& \Longleftrightarrow \mu^{\prime} \cup\left(1^{|\lambda|-|\omega|}\right) \leq \eta^{\prime}
\end{aligned}
$$

where we stress that $\mu+(|\lambda|-|\omega|)$ stands for the partition $\left(\mu_{1}+|\lambda|-|\omega|, \mu_{2}, \mu_{3}, \ldots\right)$. It is immediate that (2) implies (3). Finally, (2) follows from (3) since $\left(1^{|\lambda|-|\omega|}\right)$ is dominated by any partition (and in particular by the partition $\left(\eta_{1}^{\prime}+\cdots+\eta_{\ell+1}^{\prime}-\right.$ $\left.|\mu|, \eta_{\ell+2}^{\prime}, \eta_{\ell+3}^{\prime}, \ldots\right)$, where $\left.\ell=\ell\left(\mu^{\prime}\right)\right)$.

The following proposition was essential to deduce Theorem 2 from Theorem 1, and thus to connect the Macdonald polynomials in superspace to the double Macdonald polynomials.

Proposition 32 Suppose that $\Lambda \leftrightarrow(\lambda, \mu)$ and $\Omega \leftrightarrow(\omega, \eta)$, with $m \geq n=|\lambda|+|\mu|=$ $|\omega|+|\eta|$. Then

$$
(\lambda, \mu) \geq(\omega, \eta) \Longleftrightarrow \Lambda \geq \Omega
$$

Proof For the purpose of this proof, we first modify our convention for the insertion of the boxes of $\lambda$ and $\mu$ within $\Lambda$ into a prescription that describes $\Lambda^{*}$ built from the core $\delta^{m}$. Reconsider example (7.3) but now with $m=7$ and identify the $\circ$ and $\bullet$ as the upper and lower boxes, respectively, that lie outside the subdiagram $\delta^{m}$. This yields
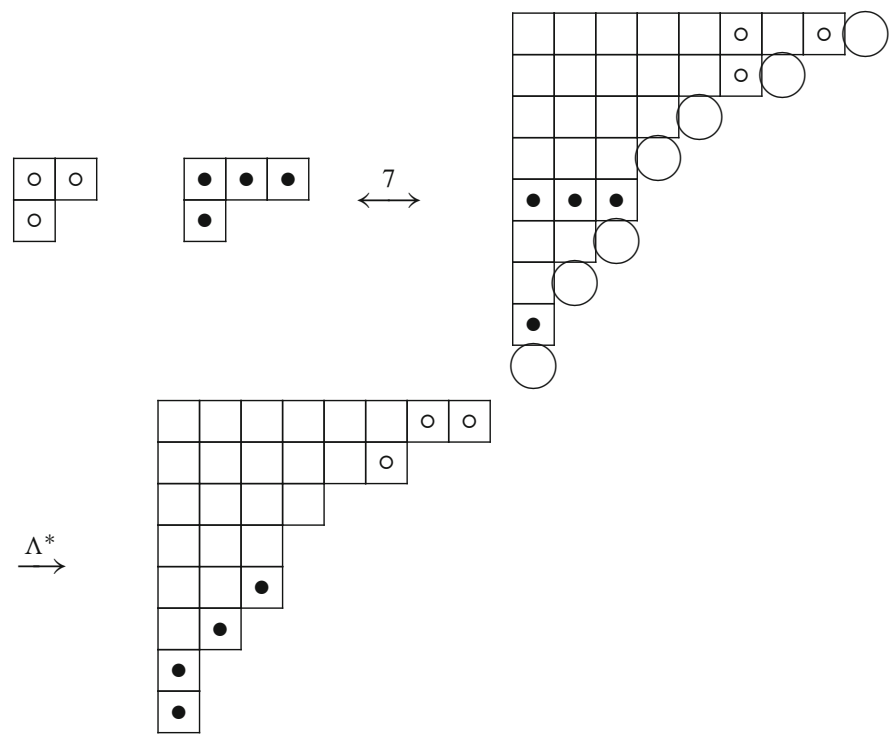
where the unmarked boxes in $\Lambda^{*}$ are those of $\delta^{7}$. More generally, $\Lambda^{*}$ can be obtained by adding $\lambda$ (resp. $\mu^{\prime}$ ) to the top rows (resp. leftmost columns) of $\delta^{m}$. In other words,

$$
\Lambda^{*}=\left(\delta^{m}+\mu\right)^{\prime}+\lambda \text {. }
$$

It is clear from the above diagrams that the row of $\Lambda$ that corresponds to the first row of $\mu$ (namely, the $\left(m+1-\mu_{1}\right)$-th row) is also the highest row in $\Lambda^{*}$ containing a •. Therefore, Lemmas 28 and 29 imply that if $m \geq n$ and $\Lambda \leftrightarrow(\lambda, \mu)$, then $\Lambda^{*}$ can always be described as above, in particular, with the $\circ$ and $\bullet$ separated from each others. In the context of this proof, $\Lambda^{*}$ will always stand for the diagrammatic representation (7.6). The following elementary observation will be fundamental.

OBS 1: Suppose that the highest $\bullet$ in $\Lambda^{*}$ lies in row $r$ and column $m+1-r$ (the highest $\bullet$ is always alone in its row by construction). If $i<r$, then the number of o's strictly below row $i$ is not larger than the number of rows $(r-i-1)$ between rows $i$ and $r$. Similarly, if $i<r$, then the number of o's strictly to the left of column $m+1-i$ is not larger than the number of columns $(r-i-1)$ between columns $m+1-i$ and $m+1-r$.

This is seen as follows. The number of o below the $i$-th row of $\Lambda^{*}$ is $\lambda_{i+1}+\cdots+\lambda_{\ell}$, where $\ell=\ell(\lambda)$. The statement is that

$$
\lambda_{i+1}+\ldots+\lambda_{\ell} \leq r-i-1=m+1-\mu_{1}-i-1,
$$

or equivalently, that

$$
\mu_{1}+i+\lambda_{i+1}+\cdots+\lambda_{\ell} \leq m
$$

But since

$$
\mu_{1}+i+\lambda_{i+1}+\cdots+\lambda_{\ell} \leq|\mu|+|\lambda|=n \leq m,
$$

the result follows. The column case is treated in a similar way, the number of $\circ$ at the left of the column $m+1-i$ being $\leq 1+\lambda_{i+2}+\ldots+\lambda_{\ell}$.

First, we show that given our hypotheses, the following implication holds:

$$
(\lambda, \mu) \geq(\omega, \eta) \Longrightarrow \Lambda \geq \Omega \text {. }
$$

The main step toward that goal is to establish that

$$
(\lambda, \mu) \geq(\omega, \eta) \Longrightarrow \Lambda^{*} \geq \Omega^{*} .
$$

We proceed by contradiction. Suppose that $(\lambda, \mu) \geq(\omega, \eta)$ and $\Lambda^{*} \nsucceq \Omega^{*}$, and let $i$ be the highest row such that

$$
\Lambda_{1}^{*}+\cdots+\Lambda_{i}^{*}<\Omega_{1}^{*}+\cdots+\Omega_{i}^{*}
$$

Note that given these conditions, we have necessarily $\Omega_{i}^{*}>\Lambda_{i}^{*}$. 
From the first condition in (1.8) and the construction (7.6) of $\Lambda^{*}$, we can conclude that $i>\ell(\omega)$. Suppose that $i$ lies above the highest $\bullet$ in $\Lambda^{*}$ (which we assume lies in a certain row $r$ ). Let $d$ be the number of $\circ$ in $\Lambda^{*}$ strictly to the left of column $m+1-i$, so that $d \leq \lambda_{i+1}+\cdots+\lambda_{\ell} \leq|\lambda|-|\omega|$. Since $d \leq|\lambda|-|\omega|$, we can use Lemma 31 to deduce

$$
\mu_{1}^{\prime}+\cdots+\mu_{\mu_{1}}^{\prime}+1+\cdots+1=\left|\mu^{\prime}\right|+d \leq \eta_{1}^{\prime}+\cdots+\eta_{\mu_{1}+d}^{\prime} .
$$

On the other hand, we have $d \leq r-i-1=m-\mu_{1}-i$ by OBS 1 , which implies that the length $\left(\mu_{1}+d\right)$ of the partition $\mu^{\prime} \cup 1^{d}$ is smaller than $m+1-i$ (and thus the $\bullet$ 's corresponding to $\eta_{1}^{\prime}, \ldots, \eta_{\mu_{1}+d}^{\prime}$ are all below row $i$ ). From (7.10) and $\left|\Lambda^{*}\right|=\left|\Omega^{*}\right|$, we also get that below row $i$ there are more cells of $\Lambda^{*}$ than cells of $\Omega^{*}$. Therefore,

$$
\left|\mu^{\prime}\right|+d>\text { number of cells of } \eta \text { below row } i \geq \eta_{1}^{\prime}+\cdots+\eta_{\mu_{1}+d}^{\prime},
$$

which leads to a contradiction (compare (7.11) and (7.12)).

Now suppose that $i$ does not lie above the highest $\bullet$ in $\Lambda^{*}$, and let $\ell=\Lambda_{i+1}^{*}$. In this case, due to the hypothesis $(\lambda, \mu) \geq(\omega, \eta)$ (which implies in particular the third expression in Lemma 31), we get

$$
\mu_{1}^{\prime}+\cdots+\mu_{\ell}^{\prime} \leq \eta_{1}^{\prime}+\cdots+\eta_{\ell}^{\prime}
$$

According to the working hypothesis we want to contradict, namely $\Omega_{i}^{*}>\Lambda_{i}^{*}$, there must again be below row $i$ more cells of $\Lambda^{*}$ than of $\Omega^{*}$. Hence, $($ letting $\ell-(i+1)=s)$

$$
\begin{aligned}
& \mu_{1}^{\prime}+\cdots+\mu_{\ell}^{\prime}-s(s-1) / 2>\text { number of cells of } \eta \text { below row } i \\
& \geq \eta_{1}^{\prime}+\cdots+\eta_{\ell}^{\prime}-s(s-1) / 2,
\end{aligned}
$$

where $s(s-1) / 2$ is the number of cells of $\eta^{\prime}$ and $\mu^{\prime}$ above row $i+1$ up to column $\ell$ (note that the condition $\Omega_{i}^{*}>\Lambda_{i}^{*}$ ensures that this number is the same for $\mu^{\prime}$ and $\eta^{\prime}$ ). Comparing (7.13) and (7.14) leads again to a contradiction, and we can conclude that (7.9) holds.

Finally, observe that

$$
\Lambda^{*}=\left(\lambda+\delta^{m}\right) \cup \mu \text { and } \Lambda^{\circledast}=\left(\lambda+\delta^{m}+1^{m}\right) \cup \mu=\left(\lambda+\delta^{m+1}\right) \cup \mu,
$$

that is, $\Lambda^{\circledast}$ corresponds to $\Lambda^{*}$ with $m$ replaced by $m+1$. But (7.9) also holds for $m+1$ (it holds for all $m \geq n$ ), which means that

$$
(\lambda, \mu) \geq(\omega, \eta) \Longrightarrow \Lambda^{*} \geq \Omega^{*} \text { and } \Lambda^{\circledast} \geq \Omega^{\circledast}
$$

and (7.8) follows.

We now need to show the reverse implication:

$$
(\lambda, \mu) \geq(\omega, \eta) \Longleftarrow \Lambda \geq \Omega .
$$


It is sufficient to show that given our hypotheses

$$
(\lambda, \mu) \geq(\omega, \eta) \Longleftarrow \Lambda^{\circledast} \geq \Omega^{\circledast} .
$$

First, we show that $\Lambda^{\circledast} \geq \Omega^{\circledast}$ implies $\lambda \geq \omega$. From (7.15), we get that the diagram of $\Lambda^{\circledast}$ is that of $\Lambda^{*}$ with $m$ replaced by $m+1$. From this construction, we have immediately (using $\ell=\ell(\lambda)$ )

$$
\Lambda^{\circledast} \geq \Omega^{\circledast} \Longrightarrow \lambda_{1}+\cdots+\lambda_{\ell} \geq \omega_{1}+\cdots+\omega_{\ell} .
$$

Hence, $\lambda_{1}+\ldots+\lambda_{i} \geq \omega_{1}+\ldots+\omega_{i}$ will hold for all $i$ if $|\lambda| \geq|\omega|$. Suppose that $|\omega|>|\lambda|$ and let $k=|\lambda|+1$. An observation similar to OBS 1 will prove useful.

OBS 2: Suppose that the highest $\bullet$ in $\Lambda^{\circledast}$ lies in row $r$. Then, the number of o's in $\Lambda^{\circledast}$ is not larger than $r-2$.

Indeed, the number of $\circ$ is equal to $|\lambda|$, and in this case $r=m+2-\mu_{1}$. Thus $|\lambda| \leq r-2=m-\mu_{1}$ since $|\lambda|+\mu_{1} \leq|\lambda|+|\mu| \leq m$. It is then immediate that the highest $\bullet$ in $\Lambda^{\circledast}$ lies below row $k$ since OBS 2 gives $|\lambda| \leq r-2$, or equivalently, $r \geq|\lambda|+2>k$ (that is, the cells of $\mu$ do not contribute to $\Lambda^{\circledast}$ up to row $k$ ). Using

$$
\omega_{1}+\cdots+\omega_{k} \geq k=|\lambda|+1
$$

which follows from the hypothesis $|\omega|>|\lambda|$, we then get

$$
\begin{aligned}
& \Omega_{1}^{\circledast}+\cdots+\Omega_{k}^{\circledast}-k(m+1)+k(k+1) / 2=\omega_{1}+\cdots+\omega_{k} \\
& \geq|\lambda|+1>\Lambda_{1}^{\circledast}+\cdots+\Lambda_{k}^{\circledast}-k(m+1)+k(k+1) / 2,
\end{aligned}
$$

which is a contradiction to $\Lambda^{\circledast} \geq \Omega^{\circledast}$. We thus have $\lambda \geq \omega$. By conjugation, we also have $\eta \geq \mu$, and thus (7.17) holds by Lemma 31 .

\section{Appendix C: Proofs of the conjectures in the stable sector}

In this section, we recall some of our previous conjectures concerning the Macdonald superpolynomials and show that in the stable sector, they match statements that were demonstrated in this article. These conjectures are thus partly validated but we stress that they preserve their conjectural status in the non-stable sector.

Let us first recall that the Macdonald superpolynomials $P_{\Lambda}(x, \theta)=P_{\Lambda}(x, \theta ; q, t)$ are defined as in Theorem 1 by the two conditions

$P_{\Lambda}(x, \theta ; q, t)=m_{\Lambda}(x, \theta)+$ lower terms, and $\left\langle\left\langle P_{\Lambda}(x, \theta), P_{\Omega}(x, \theta)\right\rangle_{q, t} \propto \delta_{\Lambda \Omega}\right.$,

but with the scalar product for the bisymmetric power sums replaced by its superspace form:

$$
(-q)^{\left(\begin{array}{c}
m \\
2
\end{array}\right)}\left\langle\left\langle p_{\lambda, \mu}, p_{\omega, \eta}\right\rangle\right\rangle_{q, t}=\left\langle\left\langle p_{\Lambda}(x, \theta), p_{\Omega}(x, \theta)\right\rangle\right\rangle_{q, t},
$$


where $m$ is the fermionic degree of $\Lambda$. This matching factor (which comes from $q^{\left|\Lambda^{a}\right|-|\lambda|}$ ) plays no role in the orthogonality conditions, and affects only the value of the norm.

Norm and integral form

We first discuss two conjectures related to the norm and the integral version of the Macdonald superpolynomials. These are as follows.

Conjecture 33 [6] The norm of the Macdonald superpolynomial $P_{\Lambda}(x, \theta ; q, t)$ is given by

$$
\left\langle\left\langle P_{\Lambda}(x, \theta), P_{\Lambda}(x, \theta)\right\rangle\right\rangle_{q, t}=(-1)^{\left(\begin{array}{c}
m \\
2
\end{array}\right)} q^{\left|\Lambda^{a}\right|} \frac{h_{\Lambda}^{\uparrow}(q, t)}{h_{\Lambda}^{\downarrow}(q, t)},
$$

where

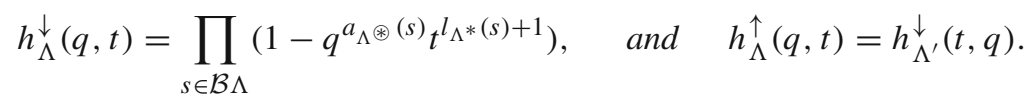

In the previous equation, $\mathcal{B} \Lambda$ stands for the subset of boxes in the diagram of $\Lambda$ whose row and column do not both end with a circle. This is referred to as the set of bosonic boxes of $\Lambda$. For instance, in Example (7.1), the bosonic boxes are $(1,3),(2,1),(2,2)$, and $(4,1)$.

Conjecture 34 [6] The integral form of the Macdonald superpolynomials $P_{\Lambda}(x, \theta$; $q, t)$ reads

$$
J_{\Lambda}(x, \theta ; q, t)=h_{\Lambda}^{\downarrow}(q, t) P_{\Lambda}(x, \theta ; q, t),
$$

where $h_{\Lambda}^{\downarrow}(q, t)$ is defined in (8.2). In other words, the monomial expansion coefficients of $J_{\Lambda}(x, \theta ; q, t)$ belong to $\mathbb{Z}[q, t]$.

In the following, we will make the connection between the conjectured formula (8.1) of the norm of the Macdonald superpolynomials and the formula (3.12) giving the norm of the double Macdonalds. In view of establishing this equivalence, we recall that the two norms differ by the factor $(-q)\left(\begin{array}{c}m \\ 2\end{array}\right)$.

Lemma 35 In the stable sector, the super and double norms are related by the equation

$$
q^{|\lambda|} \frac{h_{\Lambda}^{\uparrow}(q, t)}{h_{\Lambda}^{\downarrow}(q, t)}=b_{\lambda, \mu}(q, t)^{-1}
$$

where $\Lambda=\left(\lambda+\delta^{m} ; \mu\right)$ with $m \geq n=|\lambda|+|\mu|$. 
We observe that the r.h.s. of (8.4) is $m$-independent (it is a formula that pertains to the stable sector), while this is not obviously true for the 1.h.s. So we first establish this fact.

Lemma 36 Let $\Lambda=\left(\lambda+\delta^{m} ; \mu\right)$. Then, $h_{\Lambda}^{\downarrow}(q, t)$, defined in (8.2), does not depend upon $m$ when $m \geq n=|\lambda|+|\mu|$.

Proof When $m \geq n$, the boxes of $\Lambda$ that belong to the subdiagram $\mathcal{B} \Lambda$ are those corresponding to the inserted parts of $\lambda$ and $\mu$ in $\Lambda$ (see Appendix A). For example, with $\lambda, \mu=(3,1,1),(2,2,1)$ and $m=10$ :
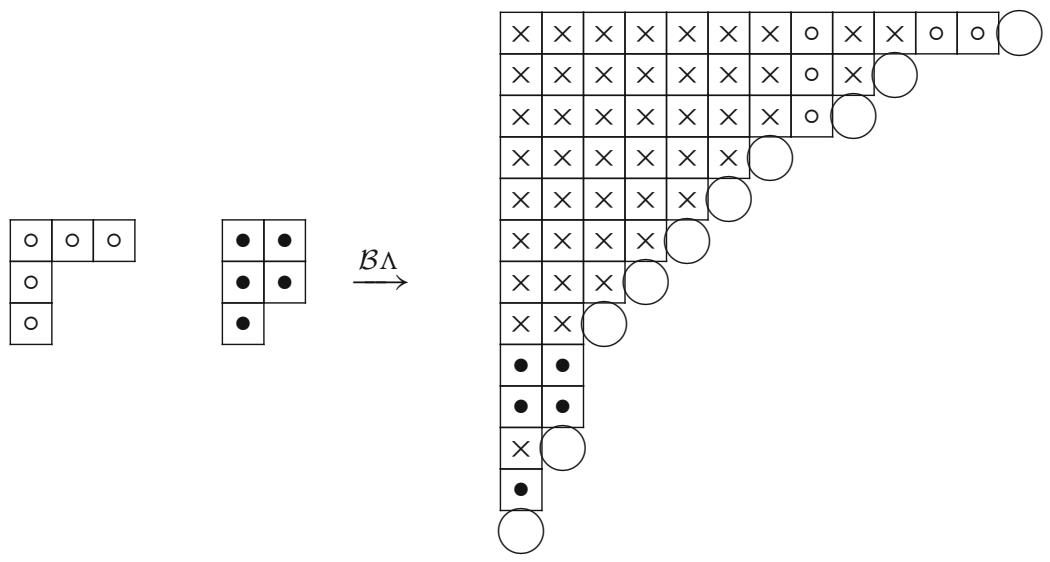

where the boxes marked with an $\times$ are not in $\mathcal{B} \Lambda$ (they lie at the intersection of a row and a column ending with a circle). Now, in the expression for $h_{\Lambda}^{\downarrow}(q, t)$, the product over the boxes of $\mathcal{B} \Lambda$ can manifestly be separated into a product over the bosonic boxes of the following two smaller superpartitions:

$$
\bar{\Omega}:=\left(\lambda+\delta^{\ell(\lambda)} ;\right) \quad \text { and } \quad \bar{\Lambda}:=\left(\delta^{\mu_{1}} ; \mu\right) .
$$

In the above example, these are
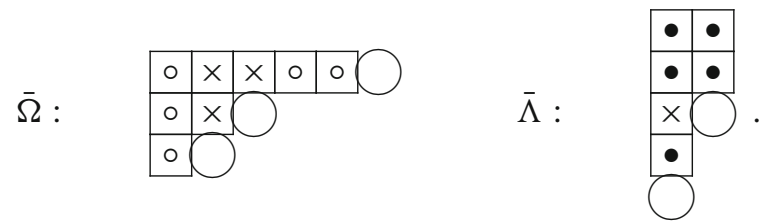

The decomposition takes the form

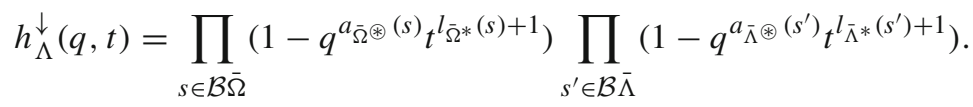

This is a simple consequence of the fact that the leg length and arm length of the corresponding boxes do not depend on the rest of the diagram (see Corollary 29). 
Now, the $m$-independence of both $\bar{\Omega}$ and $\bar{\Lambda}$ entails that of $h_{\Lambda}^{\downarrow}(q, t)$. An identical result holds for $h_{\Lambda}^{\uparrow}(q, t)$.

We now turn to the proof of Lemma 35.

Proof The objective was to rewrite the $\bar{\Omega}$ and $\bar{\Lambda}$ contributions in (8.6) solely in terms of the $\lambda$ and $\mu$ data, respectively. The first step amounts to reorganize the terms in the first product as follows:

$$
q^{a_{\bar{\Omega}^{\circledast}}(s)} t^{l_{\bar{\Omega}^{*}}(s)+1}=q^{a_{\bar{\Omega} \circledast}(s)-l_{\bar{\Omega}^{*}}(s)-1}(q t)^{l_{\bar{\Omega}^{*}}(s)+1} .
$$

Since all the parts of $\bar{\Omega}$ are fermionic, $a_{\bar{\Omega}^{\circledast}}(s)-1=a_{\bar{\Omega}^{*}}(s)$. Note also that the boxes of $\mathcal{B} \bar{\Omega}$ correspond exactly to those of $\lambda$. Then, it is easy to see that $l_{\bar{\Omega}^{*}}(s)=l_{\lambda}(r)$, where $r$ is the box corresponding to $s$ in $\lambda$. Similarly, we have $a_{\bar{\Omega}^{*}}(s)-l_{\bar{\Omega}^{*}}(s)=a_{\lambda}(r)$, the subtraction removing precisely the contribution to the arm of the non-bosonic boxes belonging to the staircase partition. We thus have

$$
\prod_{s \in \mathcal{B} \bar{\Omega}}\left(1-q^{a_{\bar{\Omega} \circledast}(s)} t^{l_{\bar{\Omega}^{*}}(s)+1}\right)=\prod_{r \in \lambda}\left(1-q^{a_{\lambda}(r)}(q t)^{l_{\lambda}(r)+1}\right) .
$$

Similarly, we reorganize the contribution of $\bar{\Lambda}$ in (8.6) as follows:

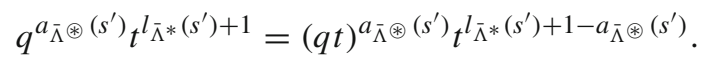

Since the rows of $\mu$, when inserted into the staircase partition, constitute rows by themselves (non-fermionic ones), we obviously have $a_{\bar{\Lambda} \circledast}\left(s^{\prime}\right)=a_{\mu}\left(r^{\prime}\right)$ with $r^{\prime}$ the box of $\mu$ corresponding to $s^{\prime}$. Arguing as before, we also have $l_{\bar{\Lambda}^{*}}\left(s^{\prime}\right)-a_{\bar{\Lambda}^{\circledast}}\left(s^{\prime}\right)=l_{\mu}\left(r^{\prime}\right)$, so that

$$
\prod_{s^{\prime} \in \mathcal{B} \bar{\Lambda}}\left(1-q^{a_{\bar{\Lambda} \circledast\left(s^{\prime}\right)}} t^{\left.l_{\bar{\Lambda}^{*}\left(s^{\prime}\right)+1}\right)}=\prod_{r^{\prime} \in \mu}\left(1-(q t)^{a_{\mu}\left(r^{\prime}\right)} t^{l_{\mu}\left(r^{\prime}\right)+1}\right) .\right.
$$

Collecting these results yield

$$
h_{\Lambda}^{\downarrow}(q, t)=\prod_{r \in \lambda}\left(1-q^{a_{\lambda}(r)}(q t)^{l_{\lambda}(r)+1}\right) \prod_{r^{\prime} \in \mu}\left(1-(q t)^{a_{\mu}\left(r^{\prime}\right)} t^{l_{\mu}\left(r^{\prime}\right)+1}\right)=c_{\lambda}(q, q t) c_{\mu}(q t, t) .
$$

A similar expression holds for $h_{\Lambda}^{\uparrow}(q, t)$ :

$$
h_{\Lambda}^{\uparrow}(q, t)=h_{\Lambda^{\prime}}^{\downarrow}(t, q)=c_{\mu^{\prime}}(t, q t) c_{\lambda^{\prime}}(q t, q)
$$

where we used $\Lambda^{\prime}=\left(\mu^{\prime}+\delta^{m} ; \lambda^{\prime}\right)$ (see Lemma 30). The expressions for the norm can thus be related as 
$q^{|\lambda|} \frac{h_{\Lambda}^{\uparrow}(q, t)}{h_{\Lambda}^{\downarrow}(q, t)}=q^{|\lambda|} \frac{c_{\lambda^{\prime}}(q t, q)}{c_{\lambda}(q, q t)} \frac{c_{\mu^{\prime}}(t, q t)}{c_{\mu}(q t, t)}=q^{|\lambda|} b_{\lambda}(q, q t)^{-1} b_{\mu}(q t, t)^{-1}=b_{\lambda, \mu}(q, t)^{-1}$.

Lemma 35 readily implies that

Corollary 37 Conjecture 33 is true for $m \geq n$.

On the other hand, the relation (8.7) implies the equivalence of the integral forms (8.3) and (3.29):

Corollary 38 Conjecture 34 is true for $m \geq n$.

\section{Kostka coefficients}

Let $\varphi$ be the endomorphism of $\mathbb{Q}(q, t)\left[p_{1}, p_{2}, p_{3}, \ldots ; \tilde{p}_{0}, \tilde{p}_{1}, \tilde{p}_{2}, \ldots\right]$ whose action on the power sums is

$$
\varphi\left(p_{n}\right)=\frac{1}{\left(1-t^{n}\right)} p_{n} \quad \text { and } \quad \varphi\left(\tilde{p}_{n}\right)=\tilde{p}_{n}
$$

We then define the modified Macdonald polynomials in superspace as

$$
H_{\Lambda}(x, \theta ; q, t)=\varphi\left(J_{\Lambda}(x, \theta ; q, t)\right) .
$$

We also introduce the Schur superpolynomial as the limit $q=t=0$ of the Macdonald superpolynomial

$$
s_{\Lambda}(x, \theta)=P_{\Lambda}(x, \theta ; 0,0) .
$$

Conjecture 39 [6] The coefficients $K_{\Omega \Lambda}(q, t)$ in the expansion of the modified Macdonald superpolynomials

$$
H_{\Lambda}(x, \theta ; q, t)=\sum_{\Omega} K_{\Omega \Lambda}(q, t) s_{\Omega}(x, \theta)
$$

are polynomials in $q$ and $t$ with non-negative integer coefficients.

When $m \geq n$, the Kostka coefficient $K_{\Omega \Lambda}(q, t)$ is equal to the coefficient $K_{\kappa, \gamma} \lambda, \mu(q, t)$ defined in (1.13) (where $\Lambda \leftrightarrow(\lambda, \mu)$ and $\Omega \leftrightarrow(\kappa, \gamma)$ ) since the transformation of the previous equation into its bisymmetric form does not affect its expansion coefficients. Therefore, as a direct consequence of Proposition 12, we have

Corollary 40 Conjecture 39 is true for $m \geq n$. 
Finally, the following symmetries of the coefficients $K_{\Omega \Lambda}(q, t)$ have been observed [7, Sect. 7.1]:

$$
K_{\Omega \Lambda}(q, t)=K_{\Omega^{\prime} \Lambda^{\prime}}(t, q) \quad \text { and } \quad K_{\Omega \Lambda}(q, t)=q^{\bar{n}\left(\Lambda^{\prime}\right)} t^{\bar{n}(\Lambda)} K_{\Omega^{\prime} \Lambda}\left(q^{-1}, t^{-1}\right)
$$

where $\bar{n}(\Lambda)$ is given by

$$
\bar{n}(\Lambda)=n(\mathcal{S} \Lambda)-d^{\mathcal{B}}(\Lambda) \quad \text { with } \quad n(\lambda)=\sum_{i}(i-1) \lambda_{i}
$$

In the previous equation, $\mathcal{S} \Lambda$ is the skew diagram $\mathcal{S} \Lambda=\Lambda^{\circledast} / \delta^{m+1}$ (we consider that $n(\lambda / \mu)=n(\lambda)-n(\mu))$. The term $d^{\mathcal{B}}(\Lambda)$ is defined as follows: fill each square $s \in \mathcal{B} \Lambda$ (we recall that $\mathcal{B} \Lambda$ was defined after Conjecture 33) with the number of boxes above $s$ that are not in $\mathcal{B} \Lambda$. Then, $d^{\mathcal{B}}(\Lambda)$ is the sum of these entries. For instance, considering example (7.1), and marking again by an $\times$ the boxes in $\Lambda$ that are not in $\mathcal{B} \Lambda$, we have

$$
d^{\mathcal{B}}((3,1,0 ; 2,1))=4:
$$

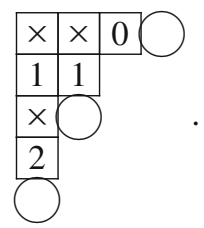

As previously mentioned, in the stable sector, $K_{\Omega \Lambda}(q, t)=K_{\kappa, \gamma} \lambda, \mu(q, t)$ with $\Lambda=\left(\lambda+\delta^{m} ; \mu\right)$ and $\Omega=\left(\kappa+\delta^{m} ; \gamma\right)$. The symmetries (8.8) must thus, in the stable sector, be a consequence of Corrolary 14 . This is immediate for the first one. In the following lemma, we show that this is also true for the second one by establishing the equivalence of the expressions $\bar{n}(\Lambda)$ and $\bar{n}(\lambda, \mu)$ (the latter defined in Corollary 14) when $m \geq n$.

Lemma 41 For $\Lambda=\left(\lambda+\delta^{m} ; \mu\right)$ and $m \geq n=|\lambda|+|\mu|$, we have

$$
n(\lambda)+|\mu|+n(\mu)+n\left(\mu^{\prime}\right)=n(\mathcal{S} \Lambda)-d^{\mathcal{B}}(\Lambda) .
$$

Proof First, consider the expression $n(\mathcal{S} \Lambda)$. Since $\mathcal{S} \Lambda=\Lambda^{\circledast} / \delta^{m+1}=\left(\left(\lambda+\delta^{m+1}\right) \cup\right.$ $\mu) / \delta^{m+1}$, the expression $n(\mathcal{S} \Lambda)$ is the "depth-weighted sum" $n(\cdot)$ (referring to $i-1$ as the depth of a box in row $i$ ) of the boxes of the diagrams of $\lambda$ and $\mu$ when inserted in the staircase partition $(m, m-1, \ldots, 0)$. For the analysis of $n(\mathcal{S} \Lambda)$, it is convenient to view the insertion of the diagrams of $\lambda$ and $\mu$ into that of $\delta^{m+1}$ to be as described in the proof of Proposition 32 in Appendix B. Consider again the example of $\lambda, \mu=$ $(3,1,1),(2,2,1)$ and $m=10$. We have 


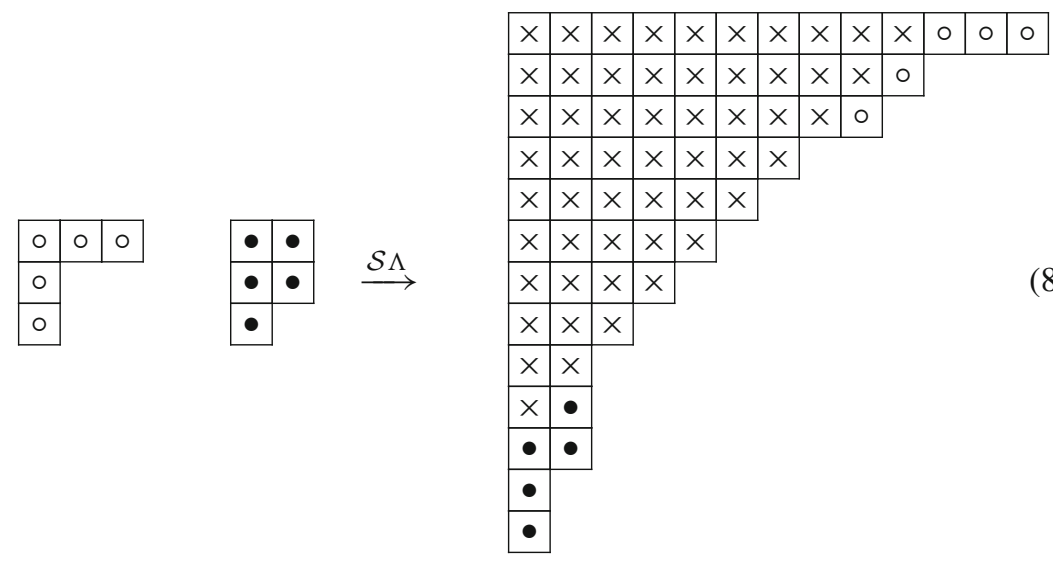

The boxes marked with an $\times$ are not considered in the evaluation of $n(\mathcal{S} \Lambda)$. Clearly, we have

$$
n(\mathcal{S} \Lambda)=n(\lambda)+G_{m}(\mu)
$$

where $G_{m}(\mu)$ is some contribution that depends on the diagram of $\mu$ and on $m$. For a general partition $\mu$ inserted into $\delta^{m+1}$ (from the bottom), we can compute $G_{m}(\mu)$ as follows. First observe that the boxes of $\mu$ are ordered as

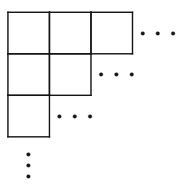

$\stackrel{G_{m}(\mu)}{\longrightarrow}$

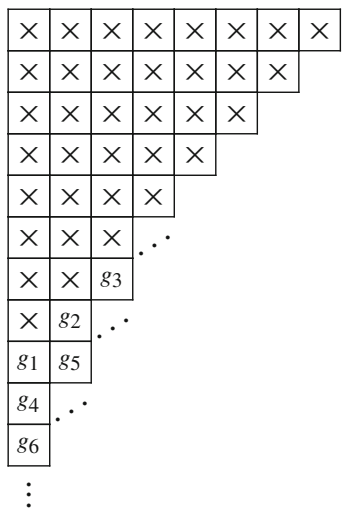

The first box $g_{1}$ is located in the $(m+1)$-th row of the total diagram. Consequently, it has depth $g_{1}=m$. Similarly, we have

$$
\begin{aligned}
& g_{2}=(m-1), \quad g_{3}=(m-2), \quad g_{4}=(m+1), \quad g_{5}=(m+1-1), \\
& g_{6}=(m+2), \ldots
\end{aligned}
$$

One easily sees the general pattern: 


$$
\begin{aligned}
G_{m}(\mu)= & (m+1-1)+(m+1-2)+\cdots+\left(m+1-\mu_{1}\right) \\
& +(m+2-1)+(m+2-2)+\cdots+\left(m+2-\mu_{2}\right) \\
& +\cdots \\
& +(m+\ell(\mu)-1)+(m+\ell(\mu)-2)+\cdots+\left(m+\ell(\mu)-\mu_{\ell(\mu)}\right) \\
= & \sum_{i=1}^{\ell(\mu)} \sum_{j=1}^{\mu_{i}}(m+i-j) .
\end{aligned}
$$

This summation is readily evaluated

$$
\begin{aligned}
G_{m}(\mu) & =\sum_{i}\left((m+i) \mu_{i}-\mu_{i}\left(\mu_{i}+1\right) / 2\right) \\
& =\sum_{i}\left((i-1) \mu_{i}+m \mu_{i}-\mu_{i}\left(\mu_{i}-1\right) / 2\right)=n(\mu)+m|\mu|-n\left(\mu^{\prime}\right),
\end{aligned}
$$

where we used the relation $\sum_{i} \mu_{i}\left(\mu_{i}-1\right) / 2=n\left(\mu^{\prime}\right)$. We have thus

$$
n(\mathcal{S} \Lambda)=n(\lambda)+n(\mu)+m|\mu|-n\left(\mu^{\prime}\right) .
$$

Let us turn to the contribution of $d^{\mathcal{B}}(\Lambda)$. For its computation, we need to return to the standard insertion of $\lambda$ and $\mu$ within $\Lambda$ illustrated in (7.3). Now, $d^{\mathcal{B}}(\Lambda)$ can naturally be split in two parts

$$
d^{\mathcal{B}}(\Lambda)=d_{1}^{\mathcal{B}}(\lambda)+d_{2}^{\mathcal{B}}(\mu)
$$

simply because all the boxes of $\mathcal{B} \Lambda$ are those corresponding to the inserted ones of $\lambda$ and $\mu$. Clearly, no box of the subdiagram $\Lambda / \mathcal{B} \Lambda$ lies above the boxes corresponding to $\lambda$ in $\mathcal{B} \Lambda$ since the latter are all inserted in the topmost rows. Consequently, we have $d_{1}^{\mathcal{B}}(\lambda)=0$. Now, consider the first row $\mu_{1}$ of $\mu$. This row is inserted in the ( $\left.m-\mu_{1}+1\right)$-th row of $\Lambda$ (see (7.3) and (7.5) for instance). Each box of this row of length $\mu_{1}$ has thus exactly $m-\mu_{1}$ boxes of $\Lambda / \mathcal{B} \Lambda$ above it, for a contribution of $\left(m-\mu_{1}\right)\left(\mu_{1}\right)$ to $d_{2}^{\mathcal{B}}(\mu)$. For the next row of $\mu$, two cases are possible. If $\mu_{2}=\mu_{1}$, then this second row of $\mu$ is just below the row $\mu_{1}$ in the diagram $\Lambda$, in which case its contribution to $d_{2}^{\mathcal{B}}(\mu)$ is $\left(m-\mu_{2}\right)\left(\mu_{2}\right)$. Otherwise $\mu_{2}<\mu_{1}$, so that this row $\mu_{2}$ is inserted in the $\left(m-\mu_{2}+1+1\right)$-th row of $\Lambda$. Therefore, the number of boxes of $\Lambda / \mathcal{B} \Lambda$ that lies above each box of this row is $\left(m-\mu_{2}+2-1-1\right)$, since we have to remove the box belonging to row $\mu_{1}$. Its contribution is then also $\left(m-\mu_{2}\right)\left(\mu_{2}\right)$. In general, the contribution of the part $\mu_{k}$ to $d_{2}^{\mathcal{B}}(\mu)$ is

[(position of the row $\mu_{k}$ in the diagram $\left.\Lambda\right)-1-\left(\#\right.$ of rows of $\mu$ above $\left.\left.\mu_{k}\right)\right] \times\left(\mu_{k}\right)$ $=\left(\left(m-\mu_{k}+k\right)-1-(k-1)\right)\left(\mu_{k}\right)=\left(m-\mu_{k}\right) \mu_{k}$. 
We thus have

$$
d^{\mathcal{B}}(\Lambda)=d_{2}^{\mathcal{B}}(\mu)=\sum_{i}\left(m-\mu_{i}\right) \mu_{i}=(m-1)|\mu|-2 n\left(\mu^{\prime}\right) .
$$

All together, this gives

$$
n(\mathcal{S} \Lambda)-d^{\mathcal{B}}(\Lambda)=n(\lambda)+n(\mu)+|\mu|+n\left(\mu^{\prime}\right) .
$$

Evaluation

In [6], we have formulated an intriguing conjecture for the evaluation of the Macdonald superpolynomials. Let $F(x, \theta)$ be a symmetric polynomial in superspace with fermionic degree $m$ and suppose that $N \geq m$. The evaluation of $F(x, \theta)$ is defined as

$$
\mathrm{E}_{N, m}[F(x, \theta)]:=\left[\frac{\partial_{\theta_{m}} \ldots \partial_{\theta_{1}} F(x, \theta)}{\Delta\left(x_{1}, \ldots, x_{m}\right)}\right]_{x_{1}=u_{1}, \ldots, x_{N}=u_{n}},
$$

where the values $u_{i}$ are given by

$$
u_{i}=\frac{t^{i-1}}{q^{\max (m-i, 0)}}
$$

and where $\Delta\left(x_{1}, \ldots, x_{m}\right)=\prod_{1 \leq i<j \leq m}\left(x_{i}-x_{j}\right)$ is the Vandermonde determinant in the variables $x_{1}, \ldots, x_{m}$. The conjectured formula involves the quantity $d^{\mathcal{F}}(\Lambda)$ defined as follows. Fill each square $s \in \mathcal{F} \Lambda$ (these are the boxes whose column and row both end with a circle) with the number of boxes above $s$ that are not in $\mathcal{F} \Lambda$. Then, $d^{\mathcal{F}}(\Lambda)$ is the sum of these entries. For instance, considering again the example (7.1), and marking by $\times$ the boxes in $\Lambda$ that are not in $\mathcal{F} \Lambda$, we have

$$
d^{\mathcal{F}}((3,1,0 ; 2,1))=1:
$$

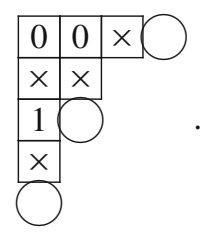

Conjecture 42 Let $\Lambda$ be of fermionic degree $m$ and suppose that $N \geq \ell\left(\Lambda^{\circledast}\right)$. Then, the evaluation formula for the Macdonald superpolynomial $P_{\Lambda}(x, \theta ; q, t)$ is

$$
\mathrm{E}_{N, m}\left[P_{\Lambda}(x, \theta ; q, t)\right]=\frac{t^{n(\mathcal{S} \Lambda)+d^{\mathcal{F}}(\Lambda)}}{q^{(m-1)\left|\Lambda^{a} / \delta^{m}\right|-n\left(\Lambda^{a} / \delta^{m}\right)} h_{\Lambda}^{\downarrow}(q, t)} \prod_{s \in \mathcal{S} \Lambda}\left(1-q^{a^{\prime} \Lambda^{\circledast}(s)} t^{N-l^{\prime} \Lambda^{\circledast}(s)}\right),
$$


where $h_{\Lambda}^{\downarrow}(q, t)$ is defined in (8.2), while $a^{\prime}(s)$ and $l^{\prime}(s)$ are the arm colength and leg colength defined in (2.2).

We now show that in the stable sector, this evaluation formula agrees with the one given in (3.28).

Lemma 43 For $\Lambda=\left(\delta^{m}+\lambda ; \mu\right)$ and $m \geq n=|\lambda|+|\mu|$, we have

$$
E_{N, m}\left(P_{\lambda, \mu}(x, y ; q, t)\right)=\mathrm{E}_{N, m}\left[P_{\Lambda}(x, \theta ; q, t)\right] .
$$

Proof The product over $\mathcal{S} \Lambda$ in (8.15) can be separated into two contributions: the boxes of the diagram of $\lambda$ and those of the diagram of $\mu$ (see for example (8.11)):

$$
\prod_{s \in\left(\delta^{m+1}+\lambda\right) / \delta^{m+1}}\left(1-q^{a_{\Lambda \circledast}^{\prime}(s)} t^{N-l_{\Lambda \circledast}^{\prime}(s)}\right) \prod_{s^{\prime} \in\left(\delta^{m+1}+\mu\right)^{\prime} / \delta^{m+1}}\left(1-q^{a_{\Lambda \circledast}^{\prime}\left(s^{\prime}\right)} t^{N-l_{\Lambda}^{\prime}{ }^{\prime}\left(s^{\prime}\right)}\right) .
$$

For the product corresponding to the diagram of $\lambda$, we have $a_{\Lambda_{\circledast}}^{\prime}(s)=m-l_{\lambda}^{\prime}(s)+a_{\lambda}^{\prime}(s)$ since a box in row $i$ of $\lambda$ has $(m-i+1)$ extra boxes to its left when considered within $S \Lambda$ (and $\left.l_{\lambda}^{\prime}(s)=i-1\right)$. Because there are no boxes above the rows of $\lambda$, we have $l_{\Lambda \circledast}^{\prime}(s)=l_{\lambda}^{\prime}(s)$. As for the product over the boxes of $\mu$, observe that the topmost box $s$ of each column of $\mu$ (into $\mathcal{S} \Lambda$ ) has exactly $m-a_{\mu}^{\prime}(s)$ boxes above it (i.e., $m-j+1$ if $s$ is in the $j$-th column and $\left.a^{\prime}(s)=j-1\right)$. Thus, for $s^{\prime} \in\left(\delta^{m+1}+\mu\right)^{\prime} / \delta^{m+1}$, we have $l_{\Lambda \circledast}^{\prime}\left(s^{\prime}\right)=l_{\mu}^{\prime}\left(s^{\prime}\right)+m-a_{\mu}^{\prime}\left(s^{\prime}\right)$ and since there are no boxes to the left of the cells corresponding to the diagram of $\mu$ in $\mathcal{S} \Lambda$, we can write $a_{\Lambda \circledast}^{\prime}\left(s^{\prime}\right)=a_{\mu}^{\prime}(s)$. With $\Lambda^{a} / \delta^{m}=\lambda$ and using $h_{\Lambda}^{\downarrow}(q, t)=c_{\lambda}(q, q t) c_{\mu}(q t, t)$ which holds true in the stable sector (cf. (8.7)), we have

$$
\begin{aligned}
\mathrm{E}_{N, m}\left[P_{\Lambda}(x, \theta ; q, t)\right]= & \frac{t^{n(\mathcal{S} \Lambda)+d^{\mathcal{F}}(\Lambda)}}{q^{(m-1)|\lambda|-n(\lambda)} c_{\lambda}(q, q t) c_{\mu}(q t, t)} \prod_{s \in \lambda}\left(1-q^{a_{\lambda}^{\prime}(s)+m-l_{\lambda}^{\prime}(s)} t^{N-l_{\lambda}^{\prime}(s)}\right) \\
& \prod_{s^{\prime} \in \mu}\left(1-q^{a_{\mu}^{\prime}\left(s^{\prime}\right)} t^{N+a_{\mu}^{\prime}\left(s^{\prime}\right)-m-l_{\mu}^{\prime}\left(s^{\prime}\right)}\right) .
\end{aligned}
$$

Now consider the quantity $d^{\mathcal{F}}(\Lambda)$. Clearly, it will only depend on the superpartition $\bar{\Lambda}$ defined in (8.5). In $\bar{\Lambda}$, there are $\mu_{1}-1$ fermionic rows. Every box in a fermionic row contributes equally to $d^{\mathcal{F}}(\Lambda)$, and this contribution is the number of parts in $\mu$ greater than this fermionic row. This entails the following expression:

$d^{\mathcal{F}}(\Lambda)=\sum_{i=1}^{\mu_{1}}(i-1)[$ multiplicity of parts $\geq i$ into $\mu]=\sum_{i=1}^{\mu_{1}}(i-1)\left[\operatorname{Card}\left\{j: \mu_{j} \geq i\right\}\right]$

But since $\operatorname{Card}\left\{j: \mu_{j} \geq i\right\}$ corresponds to $\mu_{i}^{\prime}$ and $\mu_{1}=\ell\left(\mu^{\prime}\right)$, we have that $d^{\mathcal{F}}(\Lambda)=$ $n\left(\mu^{\prime}\right)$. Using this result and the expression (8.13) for $n(\mathcal{S} \Lambda)$, we thus get

$$
n(\mathcal{S} \Lambda)+d^{\mathcal{F}}(\Lambda)=n(\lambda)+m|\mu|+n(\mu)
$$


Substituting this result into (8.16), we finally obtain

$$
\begin{aligned}
\mathrm{E}_{N, m}\left[P_{\Lambda}(x, \theta ; q, t)\right]= & \frac{t^{m|\mu|}(q t)^{n(\lambda)} t^{n(\mu)}}{q^{(m-1)|\lambda|}} \prod_{s \in \lambda} \frac{1-q^{a_{\lambda}^{\prime}(s)+m-l_{\lambda}^{\prime}(s)} t^{N-l_{\lambda}^{\prime}(s)}}{1-q^{a_{\lambda}(s)}(q t)^{l_{\lambda}(s)+1}} \\
& \prod_{s^{\prime} \in \mu} \frac{1-q^{a_{\mu}^{\prime}\left(s^{\prime}\right)} t^{N+a_{\mu}^{\prime}\left(s^{\prime}\right)-m-l_{\mu}^{\prime}\left(s^{\prime}\right)}}{1-(q t)^{a_{\mu}\left(s^{\prime}\right)} t^{l_{\mu}\left(s^{\prime}\right)+1}} \\
= & \frac{t^{m|\mu|}}{q^{(m-1)|\lambda|}} \prod_{s \in \lambda} \frac{(q t)^{l_{\lambda}^{\prime}(s)}\left(1-q^{a_{\lambda}^{\prime}(s)+m-l_{\lambda}^{\prime}(s)} t^{N-l_{\lambda}^{\prime}(s)}\right)}{1-q^{a_{\lambda}(s)}(q t)^{l_{\lambda}(s)+1}} \\
& \prod_{s^{\prime} \in \mu} \frac{t^{l_{\mu}^{\prime}\left(s^{\prime}\right)}\left(1-q^{a_{\mu}^{\prime}\left(s^{\prime}\right)} t^{N+a_{\mu}^{\prime}\left(s^{\prime}\right)-m-l_{\mu}^{\prime}\left(s^{\prime}\right)}\right)}{1-(q t)^{a_{\mu}\left(s^{\prime}\right)} t^{l_{\mu}\left(s^{\prime}\right)+1}} \\
= & \frac{t^{m|\mu|}}{q^{(m-1)|\lambda|}} \prod_{s \in \lambda} \frac{(q t)^{l_{\lambda}^{\prime}(s)}-q^{a_{\lambda}^{\prime}(s)}\left(q^{m} t^{N}\right)}{1-q^{a_{\lambda}(s)}(q t)^{l_{\lambda}(s)+1}} \\
& \prod_{s^{\prime} \in \mu} \frac{(t)^{l_{\mu}^{\prime}\left(s^{\prime}\right)}-(q t)^{a_{\mu}^{\prime}\left(s^{\prime}\right)}\left(t^{N-m}\right)}{1-(q t)^{a_{\mu}\left(s^{\prime}\right)} t^{l_{\mu}\left(s^{\prime}\right)+1}} \\
= & \frac{t^{m|\mu|}}{q^{(m-1)|\lambda|}} w_{\lambda}\left(q^{m} t^{N} ; q, q t\right) w_{\mu}\left(t^{N-m} ; q t, t\right),
\end{aligned}
$$

which is precisely the formula for $\mathrm{E}_{N, m}\left(P_{\lambda, \mu}(x, y ; q, t)\right)$.

Corollary 44 Conjecture 42 is true for $m \geq n$.

\section{Appendix D: Tables of Kostka coefficients}

Table $1 K_{\lambda, \mu \omega, v}(q, t)^{\prime}$ for degree $n=1$

Table $2 K_{\lambda, \mu \omega, v}(q, t)^{\prime}$ for degree $n=2$

\begin{tabular}{lll}
\hline & $(1), \emptyset$ & $\emptyset,(1)$ \\
\hline$(1), \varnothing$ & 1 & $q$ \\
$\emptyset,(1)$ & $t$ & 1 \\
\hline
\end{tabular}

\begin{tabular}{llllll}
\hline & $(2), \emptyset$ & $(1,1), \emptyset$ & $(1),(1)$ & $\emptyset,(2)$ & $\emptyset,(1,1)$ \\
\hline$(2), \varnothing$ & 1 & $q$ & $q+q^{2}$ & $q^{2}$ & $q^{3}$ \\
$(1,1), \emptyset$ & $q t$ & 1 & $q+q^{2} t$ & $q^{3} t$ & $q^{2}$ \\
$(1),(1)$ & $t$ & $t$ & $1+q t$ & $q$ & $q$ \\
$\emptyset,(2)$ & $t^{2}$ & $q t^{3}$ & $t+q t^{2}$ & 1 & $q t$ \\
$\emptyset,(1,1)$ & $t^{3}$ & $t^{2}$ & $t+t^{2}$ & $t$ & 1 \\
\hline
\end{tabular}


Table $3 K_{\lambda, \mu \omega, v}(q, t)^{\prime}$ for degree $n=3$

\begin{tabular}{llllll}
\hline & $(3), \emptyset$ & $(2,1), \emptyset$ & $(2),(1)$ & $\left(1^{3}\right), \emptyset$ & $(1,1),(1)$ \\
\hline$(3), \varnothing$ & 1 & $q+q^{2}$ & $q+q^{2}+q^{3}$ & $q^{3}$ & $q^{2}+q^{3}+q^{4}$ \\
$(2,1), \varnothing$ & $q t$ & $1+q^{2} t$ & $q+q^{2} t+q^{3} t$ & $q$ & $q+q^{2}+q^{3} t$ \\
$(2),(1)$ & $t$ & $t+q t$ & $1+q t+q^{2} t$ & $q t$ & $q+q t+q^{2} t$ \\
$\left(1^{3}\right), \varnothing$ & $q^{3} t^{3}$ & $q t+q^{2} t^{2}$ & $q^{2} t+q^{3} t^{2}+q^{4} t^{3}$ & 1 & $q+q^{2} t+q^{3} t^{2}$ \\
$(1,1),(1)$ & $q t^{2}$ & $t+q t^{2}$ & $2 q t+q^{2} t^{2}$ & $t$ & $1+q t+q^{2} t^{2}$ \\
$(1),(2)$ & $t^{2}$ & $t^{2}+q t^{3}$ & $t+2 q t^{2}$ & $q t^{3}$ & $t+q t^{2}+q^{2} t^{3}$ \\
$\emptyset,(3)$ & $t^{3}$ & $q t^{4}+q^{2} t^{5}$ & $t^{2}+q t^{3}+q^{2} t^{4}$ & $q^{3} t^{6}$ & $q t^{3}+q^{2} t^{4}+q^{3} t^{5}$ \\
$(1),(1,1)$ & $t^{3}$ & $t^{2}+t^{3}$ & $t+t^{2}+q t^{3}$ & $t^{2}$ & $t+t^{2}+q t^{2}$ \\
$\emptyset,(2,1)$ & $t^{4}$ & $t^{3}+q t^{5}$ & $t^{2}+t^{3}+q t^{4}$ & $q t^{4}$ & $t^{2}+q t^{3}+q t^{4}$ \\
$\emptyset,\left(1^{3}\right)$ & $t^{6}$ & $t^{4}+t^{5}$ & $t^{3}+t^{4}+t^{5}$ & $t^{3}$ & $t^{2}+t^{3}+t^{4}$ \\
\hline$(3), \emptyset$ & $q^{2}+q^{3}+q^{4}$ & $q^{3}$ & $q^{3}+q^{4}+q^{5}$ & $q^{4}+q^{5}$ & $q^{6}$ \\
$(2,1), \emptyset$ & $q^{2}+q^{3} t+q^{4} t$ & $q^{4} t$ & $q^{2}+q^{3}+q^{4} t$ & $q^{3}+q^{5} t$ & $q^{4}$ \\
$(2),(1)$ & $q+q^{2}+q^{2} t$ & $q^{2}$ & $q+q^{2}+q^{3} t$ & $q^{2}+q^{3}$ & $q^{3}$ \\
$\left(1^{3}\right), \varnothing$ & $q^{3} t+q^{4} t^{2}+q^{5} t^{3}$ & $q^{6} t^{3}$ & $q^{2}+q^{3} t+q^{4} t^{2}$ & $q^{4} t+q^{5} t^{2}$ & $q^{3}$ \\
$(1,1),(1)$ & $q+q^{2} t+q^{3} t^{2}$ & $q^{3} t$ & $q+2 q^{2} t$ & $q^{2}+q^{3} t$ & $q^{2}$ \\
$(1),(2)$ & $1+q t+q^{2} t^{2}$ & $q$ & $2 q t+q^{2} t^{2}$ & $q+q^{2} t$ & $q^{2} t$ \\
$\emptyset,(3)$ & $t+q t^{2}+q^{2} t^{3}$ & 1 & $q t^{2}+q^{2} t^{3}+q^{3} t^{4}$ & $q t+q^{2} t^{2}$ & $q^{3} t^{3}$ \\
$(1),(1,1)$ & $t+q t+q t^{2}$ & $q t$ & $1+q t+q t^{2}$ & $q+q t$ & $q$ \\
$\emptyset,(2,1)$ & $t+t^{2}+q t^{3}$ & $t$ & $t+q t^{2}+q t^{3}$ & $1+q t^{2}$ & $q t$ \\
$\emptyset,\left(1^{3}\right)$ & $t^{2}+t^{3}+t^{4}$ & $t^{3}$ & $t+t^{2}+t^{3}$ & $t+t^{2}$ & 1 \\
\hline & & & & &
\end{tabular}

\section{References}

1. Baker, T.H., Dunkl, C.F., Forrester, P.J.: Polynomial eigenfunctions of the Calogero-Sutherland model with exchange terms. In: van Diejen, J.F., Vinet, L. (eds.) Calogero-Moser-Sutherland Models. Springer, New York (2000)

2. Berest, Y., Etingof, P., Ginzburg, V.: Finite dimensional representations of rational Cherednik algebras. Int. Math. Res. Not. 19, 1053-1088 (2003)

3. Bergeron, F.: Algebraic combinatorics and co-invariant spaces. CMS Treatise in Mathematics. A.K. Peters Publishers, Wellesley (2009)

4. Bergeron, F., Garsia, A. M.: Science fiction and Macdonald's polynomials, Algebraic Methods and $q$-special Functions (Montreal, QC, 1996), CRM Proc. Lecture Notes, vol. 22, Amer. Math. Soc., Providence, RI, 1-52 (1999)

5. Bergeron, F., Garsia, A.M., Haiman, M., Tesler, G.: Identities and positivity conjectures for some remarkable operators in the theory of symmetric functions. Asian J. Math. 6, 103-160 (1999)

6. Blondeau-Fournier, O., Desrosiers, P., Lapointe, L., Mathieu, P.: Macdonald polynomials in superspace: conjectural definition and positivity conjectures. Lett. Math. Phys. 101, 27-47 (2012)

7. Blondeau-Fournier, O., Desrosiers, P., Lapointe, L., Mathieu, P.: Macdonald polynomials in superspace as eigenfunctions of commuting operators. J. Comb. 3(3), 495-562 (2012)

8. Bowman, C., De Visscher, M., Orellana, R.: A closed formula for the decomposition of tensor products of Specht modules for the symmetric group, arXiv:1210.5579v1 
9. Desrosiers, P., Lapointe, L., Mathieu, P.: Orthogonality of Jack polynomials in superspace. Adv. Math. 212, 361-388 (2007)

10. Desrosiers, P., Lapointe, L., Mathieu, P.: Evaluation and normalization of Jack superpolynomials. Int. Math. Res. Not. 23, 5267-5327 (2012)

11. Desrosiers, P., Lapointe, L., Mathieu, P.: Jack superpolynomials with negative fractional parameter: clustering properties and super-Virasoro ideals. Commun. Math. Phys. 316, 395-440 (2012)

12. Desrosiers, P., Lapointe, L., Mathieu, P.: Superconformal field theory and Jack superpolynomials. J. High Energy Phys. 1209, 37 (2012)

13. van Diejen, J.F.: Asymptotics of multivariate orthogonal polynomials with hyperoctahedral symmetry. Contemporary Math. 417, 157-169 (2006)

14. Garsia, A.M., Haiman, M.: A graded representation model for Macdonald polynomials. Proc. Nat. Acad. Sci. USA 99, 3607-10 (1993)

15. Gordon, I.: On the quotient ring by diagonal harmonics. Inv. Math. 153, 503-518 (2003)

16. Haglund, J.: The $q, t$-Catalan numbers and the space of diagonal harmonics. Univ. Lect. 41 AMS (2008)

17. Haiman, M.: Conjectures on the quotient ring by diagonal invariants. J. Algebr. Comb. 3(1), 17-76 (1994)

18. Haiman, M.: Hilbert schemes, polygraphs, and the Macdonald positivity conjecture. J. Am. Math. Soc. 14, 941-1006 (2001)

19. Haiman, M.: Combinatorics, symmetric functions and Hilbert Schemes. Curr. Dev. Math. 30-111 (2001)

20. Lascoux, A.: Symmetric functions and combinatorial operators on polynomials. CBMS 99, AMS (2003)

21. Macdonald, I.G.: Symmetric Functions and Hall Polynomials, 2nd edn. Clarendon Press, Oxford (1995)

22. Macdonald, I.G.: Affine Hecke algebras and orthogonal polynomials. Séminaire Bourbaki 1994-95, exposé 797, p. 189-207

23. Macdonald, I.G.: Affine Hecke Algebras and Orthogonal Polynomials. Cambridge University Press, Cambridge (2003)

24. Stump, C.: $q$, $t$-Fuß-Catalan numbers for finite reflection groups. J. Algebr. Combin. 32(1), 67-97 (2010) 